Rectangular shell elements based on the absolute nodal coordinate formulation

\title{
Department of Engineering
}




\section{Rectangular shell elements based on the absolute nodal coordinate formulation}

\section{PhD Dissertation \\ Per Hyldahl \\ July 2015}

AARHUS

UNIVERSITY

DEPARTMENT OF ENGINEERING 
ISBN: 978-87-7507-354-2

DOI: 10.7146/aul.97.90 
Thesis submitted: July 31, 2015

PhD Supervisor: Senior Associate Professor PhD Ole Balling

Department of Engineering, Aarhus University

Denmark

PhD Committee: Professor PhD José Luis Escalona Franco

Department of Mechanical and Materials Engineering, University of Seville

Spain

Professor PhD Erik Lund

Department of Mechanical and Manufacturing Engineering (M-Tech), Aalborg University

Denmark

Professor Dr. techn. Henrik Myhre Jensen

Department of Engineering, Aarhus University

Denmark

PhD Series: $\quad$ Faculty of Science \& Technology,

Aarhus University

Published by:

River Publishers

Niels Jernes Vej 10

9220 Aalborg

Denmark

http://riverpublishers.com/

(c) Copyright by Per Hyldahl

Printed in Denmark by SUN-TRYK, 2015 



\section{Abstract}

This thesis concerns the development of tools that are useful for designing machine systems and components in an virtual environment using flexible multibody dynamics. Initially, flexible multibody dynamics is briefly reviewed to explain available formalisms and possible applications of this method. Subsequently, attention is turned towards the analysis of structures that undergo large deformations and rotations using shell finite elements based on the absolute nodal coordinate formulation, ANCF. This topic is the focus for the remainder of the thesis.

The thesis is divided into three main parts. The first part acts as a general introduction to ANCF based shell finite elements and sums up available elements found in the research literature. In the end, a new ANCF element developed during this $\mathrm{PhD}$ project is presented which gives enhanced modeling capabilities of problems including e.g. moving boundary conditions.

The second part concerns the performance and behavior of a certain class of ANCF shell elements that are developed for analysis of thin shell structures. This includes discussions on differences concerning their kinematic descriptions and disclosure of certain issues regarding their performance. Those being sensitivity to irregular mesh, pour representation of curved structures and load dependent convergence when analyzing curved structures.

The final part concerns the development of a new versatile ANCF shell element. This element is distinguished by being able to describe both thin and thick curved structures. This part contains a thorough derivation of its kinematics and stiffness description, as well as numerical examples to demonstrate its performance. However, this part of the study is not yet complete.

Finally, the findings of this $\mathrm{PhD}$ project are summed up in a conclusion and possibilities for further studies and perspectives are outlined. 



\section{Resumé}

Denne afhandling omhandler udviklingen af værktøjer, som er nyttige til at designe maskiner og maskindele i et virtuelt miljø vha. fleksibel flerlegemedynamik. Indledningsvist gives der en kort gennemgang af den fleksible flerlegeme-dynamik, for at give et overblik over metoden og dens mulige anvendelser. Dernæst flyttes opmærksomheden over på analyse af strukturer som udsættes for store deformationer og rotationer vha. endelige skalelementer (eng: shell finite elements) baseret på absolutte knude koordinater (eng: absolute nodal coordinate formulation, ANCF). Dette emne vil være fokus i resten af afhandlingen.

Afhandlingen er inddelt i 3 dele. Den første del giver en general introduktion til ANCF skal-elementer, og opsummerer de forskellige elementklasser som kan findes i litteraturen. Mod slutningen introduceres der et nyt ANCF skal-element, som er udviklet under dette ph.d.-projekt. Dette element muliggør forbedret modellering af problemer med mobile randbetingelser.

Anden del omhandler effektiviteten og egenskaberne hos en særlig klasse af ANCF skal-elementer, som er udviklet specielt til analyse af tynde skalstrukturer. Heri diskuteres der forskelle i deres kinematiske beskrivelser og der fremhæves nogle uheldige egenskaber vedrørende deres adfærd. Det være sig følsomhed overfor uensartede elementstørrelser, ringe beskrivelse af kurvede strukturer og lastafhængig konvergens i forbindelse med analyse af kurvede strukturer.

Den sidste del omhandler udviklingen af et nyt alsidigt ANCF skal-element. Dette element udmærker sig ved at være anvendeligt til at beskrive både tynde og tykke skalstrukturer. Denne del indeholder en grundig udledning af elementets kinematik og stivhed, samt numeriske eksempler som demonstrerer dets effektivitet. Denne del af studiet er dog ikke færdiggjort endnu.

Slutteligt opsummeres resultaterne af ph.d.-studiet og der nævnes muligheder for fremtidige arbejder og perspektiver. 



\section{Preface}

This thesis is submitted to the Graduate School of Science and Technology (GSST) at Aarhus University in a partial fulfillment of the requirements for the degree of Doctor of Philosophy. The work has been carried out during the period from August 2011 to July 2015 at the Department of Engineering at Aarhus University.

The thesis concerns investigations within and further development of rectangular shell finite elements based on the absolute nodal coordinate formulation. The thesis is written as a monograph based on published research papers, and the papers are attached in their original form as appendices.

It has not been possible to complete this thesis without the help and support of several people, to whom I am deeply grateful. I would like to thank my supervisor Senior Associate Professor Ole Balling for considering me for the $\mathrm{PhD}$ project, and for valuable discussions and guidance during the project. Furthermore, I owe many thanks to Professor Aki Mikkola, Lappeenranta University of Technology, and Associate Professor Hiroyuki Sugiyama, University of Iowa, for hosting me during visits at their respective research groups. In that connection, special thanks go to the members of their research groups for making my stays pleasant and enjoyable. I would also like to thank Administrator Mette Stig Hansen at Aarhus University for always being helpful with practical matters and for valuable assistance with proofreading of this thesis.

My fellow PhD students at the Mechanical Engineering section, Department of Engineering Alex Møberg, Mads Krabbe, Søren Steffensen, Kennet Olesen and Jens Wind, I also owe great thanks for providing a great working environment and good company. Especially Jens Wind deserves special acknowledgment, as he has been a great 
help through daily discussions, and, in particular, in the final stages of the project by giving valuable comments and proofreading of this thesis.

Last, I would like to thank my family and friends and in particular my girlfriend, Lise, who has been very supportive at all times, and had to do without me during my long stay at University of Iowa. Thanks to all of you.

Per Hyldahl Aarhus University, July 31st, 2015 


\section{Nomenclature}

Abbreviations
ALE
Arbitrary Lagrange-Euler
ANCF Absolute nodal coordinate formulation
ANS Assumed natural strain
CID Curve induced distortion
CM Continuum mechanics
d.o.f. Degrees of freedom
EAS Enhanced assumed strain
FFRF Floating frame of reference formulation
LD Large deflection
Q8 Eight noded quadratic shell finite element
RI Reduced integration
SD Small deflection
SM Structural mechanics

\section{Matrix and vector variables}

D Constitutive matrix

E Green-Lagrange strain tensor

G Matrix of EAS polynomials

$\mathbf{K}_{T} \quad$ Tangent stiffness matrix

M Mass matrix 


$\begin{array}{ll}\mathbf{N} & \text { Matrix of shape functions } \\ \mathbf{n} & \text { Normal vector } \\ \mathbf{q} & \text { Vector of generalized coordinates for an element } \\ \mathbf{q}_{i} & \text { Vector of generalized coordinates for node } i \\ \mathbf{r} & \text { Position vector of an arbitrary point } \\ \mathbf{r}_{i} & \text { Position vector of node } i \\ \mathbf{Q}_{e} & \text { Vector of generalized elastic forces } \\ \mathbf{Q}_{f} & \text { Vector of generalized external forces } \\ \mathbf{S} & \text { Second Piola-Kirchhoff stress tensor } \\ \boldsymbol{\alpha} & \text { Vector of EAS parameters } \\ \boldsymbol{\varepsilon} & \text { Vector of in-plane normal and shear strains } \\ \boldsymbol{\gamma} & \text { Vector of transverse shear strains } \\ \boldsymbol{\kappa} & \text { Vector of midplane curvatures } \\ \lambda & \text { Vector of Lagrange multipliers } \\ \mathbf{\Phi} & \text { Vector of algebraic constraint equations }\end{array}$

\section{Latin variables}

$\begin{array}{ll}A & \text { Area } \\ E & \text { Young's modulus } \\ h & \text { Thickness } \\ L & \text { Element length } \\ t & \text { Time } \\ V & \text { Volume } \\ W & \text { Element width } \\ X, Y, Z & \text { Global coordinates } \\ x, y, z & \text { Local element coordinates }\end{array}$


Nomenclature

\section{Greek variables}
$\xi, \eta$
Isoparametric mapping parameters
V
Poisson's ratio
$\rho \quad$ Material density
$\varepsilon \quad$ Strain component

\section{Mathematical operators}
$\delta[\cdot] \quad$ Virtual operator
$\partial[\cdot] \quad$ Partial derivative operator
$[\cdot]^{T} \quad$ Matrix transpose
$[\cdot]^{-1} \quad$ Matrix inverse
$[\because] \quad$ First order time derivative
$[\because] \quad$ Second order time derivative
: $\quad$ Double dot product
$\otimes \quad$ Kronecker product

\section{Sub and superscripts}

$[\cdot]_{p} \quad$ Quantity associated to point $p$

$[\cdot]_{i} \quad$ Quantity associated to node $i$

$[\cdot]_{0} \quad$ Quantity defined in the initial configuration $i$

$[\cdot]^{(n)} \quad$ Quantity defined at iteration $n$ 
Nomenclature 


\section{Contents}

Abstract v

Resumé vii

$\begin{array}{ll}\text { Preface } & \text { ix }\end{array}$

Nomenclature $\quad$ xi

Thesis Details $\quad$ xix

Introduction 3

1 Flexible multibody dynamics . . . . . . . . . . . 4

2 Absolute nodal coordinate formulation ......... 9

3 Scope of work . . . . . . . . . . . . . . . . . 11

I Shell elements based on the ANCF 13

4 Fully parameterized shell elements . . . . . . . . . . 17

5 Gradient deficient shell elements . . . . . . . . . . . 18

6 Equations of motion ............... 20

6.1 Calculation of internal elastic forces . . . . . . 22

6.2 Static equilibrium . . . . . . . . . . . . . . . 25

6.3 Discussion . . . . . . . . . . . . 26

7 A combined ALE-ANCF shell element . . . . . . . . 27

7.1 Kinematics of ALE-ANCF based shell elements . 27

7.2 Equations of motion ................ 31

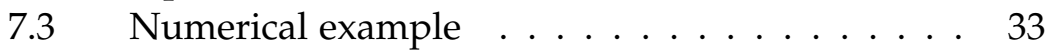

$7.4 \quad$ Discussion . . . . . . . . . . . . . 35 


\section{Behavior of thin ANCF shell elements in various mesh configurations}

8 Discussion on the kinematics of thin ANCF shell elements 42

9 Convergence study on model with optimal mesh . . . . 45

10 Influence of non-uniform element sizes on model stiffness . . . . . . . . . . . . . . . . . . . . . 49

11 Representation of initially curved structures . . . . . . 52

12 Convergence study on initially in-plane curved structures 58

13 Improved kinematics of 36 d.o.f. ANCF shell elements using element specific parameters . . . . . . . . . 62

14 Interim conclusion . . . . . . . . . . . . . . . . . . 69

\section{Shear and thickness deformable ANCF shell elements 73}

15 Parameterization of quadratic shear and thickness deformable ANCF shell element . . . . . . . . . . . . . . 77

16 Kinematics of a quadratic shear and thickness deformable ANCF shell element . . . . . . . . . . . . . . . . 79

17 Elastic forces . . . . . . . . . . . . . . . . . . . . . 81

18 Remedies for alleviating locking . . . . . . . . . 85

18.1 Reduced integration . . . . . . . . . . . 85

18.2 Assumed natural strain . . . . . . . . . . . 86

18.3 Enhanced assumed strain . . . . . . . . . . . . 88

19 Numerical examples . . . . . . . . . . . . . . . . . . . . 991

19.1 Convergence study . . . . . . . . . . . . . . . . 93

19.2 Eigenfrequency analysis . . . . . . . . . . . . 98

19.3 Assessment of curvature thickness locking . . . . 102

20 Discussion on shear deformable shell finite elements for non-linear analysis . . . . . . . . . . . . . 107

21 Interim conclusion . . . . . . . . . . . . . . . . . . . 109

$\begin{array}{ll}\text { Closing } & 117\end{array}$

22 Discussion and conclusion . . . . . . . . . . . . 117

23 Perspectives . . . . . . . . . . . . . . . . . 119

24 Afterword . . . . . . . . . . . . . . . . 122 
References 123

References . . . . . . . . . . . . . . . . 125

IV Papers

A A thin plate element based on the combined arbitrary LagrangeEuler and absolute nodal coordinate formulation

B Behavior of thin rectangular ANCF shell elements in various mesh configurations

C Studies on the kinematics of thin shell elements based on the absolute nodal coordinate formulation

D Dynamic analysis of offshore oil pipe installation using the absolute nodal coordinate formulation

E Convergence characteristics of thin ANCF shell elements in arbitrary and initially curved mesh

F Modeling and feasibility study of nonlinear suspension components in multibody systems using absolute nodal coordinate formulation based beam elements - application to stabilizer bar. 
Contents

xviii 


\section{Thesis Details}

Thesis Title: Rectangular shell elements based on the absolute nodal coordinate formulation

Ph.D. Student: Per Hyldahl

Supervisor: $\quad$ Senior Associate Professor PhD Ole Balling, Aarhus University

The main body of this thesis consist of the following papers.

[38] Hyldahl, P., Mikkola, A. M., and Balling, O. A thin plate element based on the combined arbitrary lagrange-euler and absolute nodal coordinate formulations. Proceedings of the Institution of Mechanical Engineers, Part K: Journal of Multi-body Dynamics 227 (2013), 211-219

[40] Hyldahl, P., Mikkola, A. M., Balling, O., and Sopanen, J. T. Behavior of thin rectangular ancf shell elements in various mesh configurations. Nonlinear Dynamics 78 (2014), 1277-1291

[39] Hyldahl, P., Mikкola, A. M., and Balling, O. Studies on the kinematics of thin shell elements based on the absolute nodal coordinate formulation. Proceedings of the ASME 2005 International Design Engineering Technical Conferences $\mathcal{E}$ Computers and Information in Engineering Conference (2014). Buffalo, New York, USA 
In addition to the main papers, the following publications have also been made.

[59] Nielsen, J. D., Madsen, S. B., Hyldahl, P., and Balling, O. Dynamic analysis of offshore oil pipe installation using the absolute nodal coordinate formulation. Proceedings of the ASME 2013 International Mechanical Engineering Congress E Exposition IMECE2013 (2013). San Diego, California, USA

[41] Hyldahl, P., Mikkola, A. M., Balling, O., and Sopanen, J. T. Convergence characteristics of thin ancf shell elements in arbitrary and initially curved mesh. Proceedings of The 3rd Joint International Conference on Multibody System Dynamics $\mathcal{E}$ The 7th Asian Conference on Multibody Dynamics (2014). BEXCO, Busan, Korea

[37] Hyldahl, P., Andersen, S., Mikkelsen, S., and Balling, O. Modeling and feasibility study of nonlinear suspension components in multibody systems using absolute nodal coordinate formulation based beam elements -application to stabilizer bar. SAE International Journal of Passenger Cars - Mechanical Systems 8(2) (2015)

This thesis has been submitted for assessment in partial fulfillment of the $\mathrm{PhD}$ degree. The thesis is based on the submitted or published scientific papers which are listed above. Parts of the papers are used directly or indirectly in the thesis. As part of the assessment, co-author statements have been made available to the assessment committee and are also available at the Faculty. 
Introduction 



\section{Introduction}

The design engineers of today are facing remarkable challenges when designing new products and machines. The requirements demanded by both consumers and legislators seem to be ever increasing and products must be larger, lighter, faster, and more reliable to maintain a market leading position. Additionally, the major challenge in applying for financial support for new product investments calls for higher focus on reliable risk analysis. To comply with these demands, design engineers are turning towards new and advanced methods in the design process. Exotic materials such as lightweight metal alloys and fiber reinforced polymer composites are chosen to obtain products with high stiffness to weight ratio, good aesthetic characteristics and/or low energy consumption. The latter being both in the production, the service and the decomposition phase. Advanced manufacturing processes such as rapid prototyping and robotics are used for low production cycles and customer specific products. Sophisticated simulation software is used throughout the entire design process for enhanced product insight and optimized product behavior. During the recent years, the combination of advanced simulation tools and increasing computational power has made it possible to simulate extreme events and perform numerous what-if scenarios in a virtual environment. The use of computer-aided engineering tools makes it possible to investigate the potential of any plausible design by including e.g. non-linear material descriptions, fluid-structural interaction (FSI) and large displacement formulations in numerical models. This has reduced the need for physical testing, leading to reduced time to market and safer and more reliable products.

When designing complex mechanical systems, design engineers must choose an appropriate analysis tool for the problem at hand. If 
the mechanical system is governed by large rigid-body motion rather than deformations, multibody dynamics would be an excellent choice [31, 75, 67]. Multibody dynamics is developed specifically to model the interaction between interconnected spatial bodies in the time domain. During simulation, the bodies can be subjected to complicated loading while taking into account inertia forces due to large rigid body motion. This makes it possible to efficiently analyze and optimize e.g. dynamic behavior of systems for improved load carrying capacity or performance behavior. Multibody dynamics has a proven track record of successful applications in several engineering fields including robotics, aerospace engineering, energy industry, vehicle dynamics, bio-mechanics, and rotor dynamics.

When analyzing a mechanical system, the modeling of the kinematic coupling of bodies is an essential part. The kinematic coupling of bodies is modeled using algebraic constraint equations [31, 60]. These constraints are used to model idealized joints that allow for some relative motion between bodies while eliminating others. These constraint equations are, in the general, case highly non-linear equations that are functions of both the system configuration and time. The constraint equations can be coupled with the Newton-Euler equations leading to a set of constrained equations of motion for the entire system. These sets of equations contain both algebraic and differential terms. Due to the non-linearity of both the constraint equations and the overall motion of the system, in general, there exists no analytical closed form solution to the constrained equations of motion. This calls for efficient numerical solution techniques in order to simulate system behavior over time.

\section{Flexible multibody dynamics}

Traditionally, when using multibody dynamics, the bodies have been considered rigid [31]. During the last decades, though, the multibody approach has been expanded with capabilities to include deformable bodies in order to obtain more realistic simulation models. Furthermore, the use of flexible bodies can help the designer gain detailed insight into the performance of systems where deflections of the physical components will affect the overall dynamic behavior. The incor- 


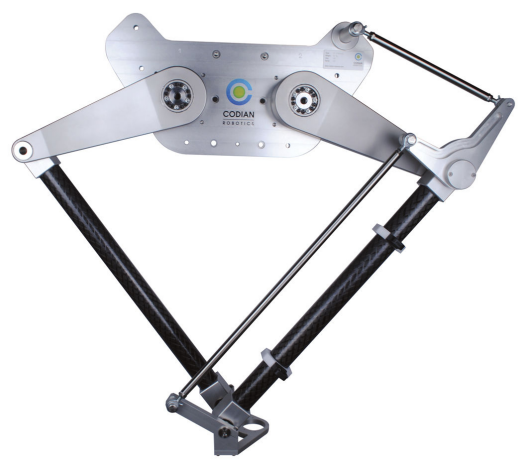

(a)

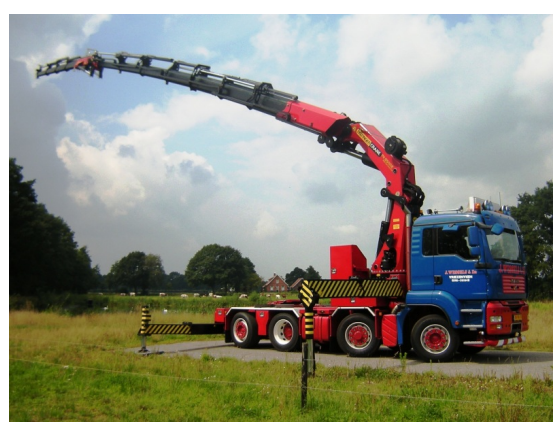

(c)

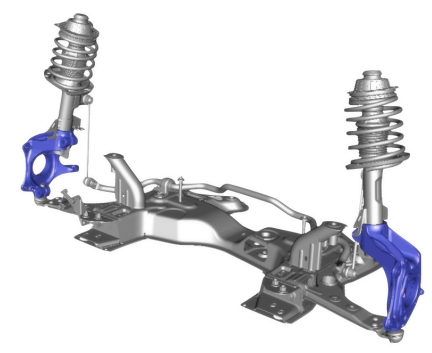

(b)

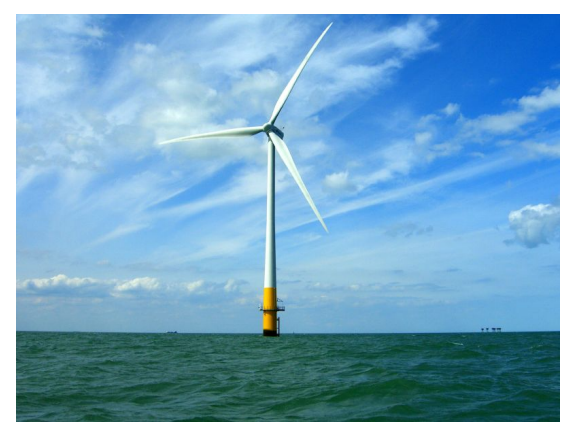

(d)

Fig. 1: Possible applications of flexible multibody dynamics. Two axis delta robot, Codian Robotics D2-1000 (a), schematics of front suspension of an 2010 Alfa Romeo Giulietta (b), Palfinger telescopic boom crane (c), and Vestas V-90 3 MW offshore wind turbine $(\mathrm{d})$.

poration of flexible bodies in the multibody dynamics framework has proven useful in many practical applications. A few examples of these are shown in Fig. 1 and described below.

High speed flexible manipulators (see Fig. 1a) are now a common part of the production line of e.g. electronic devices where they are used to position electronic components on printed circuit boards before soldering. This task requires both very high precision and speed to ensure a high throughput. Due to the fast work cycle speed, there is a risk that the mechanism will start to resonate at a certain work pace. Using flexible multibody dynamics [47], a robot can be designed such that the resonance frequencies do not coincide with the work fre- 
quency and hereby reduce the risk of resonance in the mechanism. Furthermore, a flexible multibody dynamics model can be coupled with a controller model to tune the controller parameters to obtain the most precise and efficient movement of the end effecter mounted on the robot. Such a procedure can help to reduce the changeover time between different products since programming of the robot can be done offline.

In the area of vehicle design, multibody dynamics is one of the key tools used to design and optimize suspension components (see Fig. 1b) to ensure vehicle stability and ride comfort [11]. Flexible multibody dynamics modeling of certain suspension components can be used to represent the components that can be difficult to characterize using e.g. simple linear springs [63, 89, 37]. Correct modeling of these components is crucial in order to create models that can predict the physical behavior of the vehicle to a high order of accuracy. This is important since the models can be used to determine the vehicles capability to handle sudden or hazardous maneuvers in a safe manner. Furthermore, the models can be used to extract the peak loads on key components for further analysis and design in order to produce components that do not fail unexpectedly.

In the transport and handling industry, a wide selection of cranes are used to pick up payload and carefully place it in a specific location. In the case of heavy payloads or cranes with a long reach (see Fig. 1c) the crane may deform as the load is moved, which means that flexibility must be taken into account for accurate modeling. A good multibody dynamics model of such cranes can be a valuable tool to e.g. determine the safe working envelope of the crane or simulate a difficult lift to determine and remedy possible problems before the actual lift. For optimal design of a crane's hydraulic system, coupled models of the hydraulics and the crane can be used to design hydraulic valves and control algorithms for smooth and safe operation [4]. In the case of telescopic cranes (see Fig. 1 (c)), special care must be taken in the modeling phase to correctly model the interaction between the telescopic sections which is a complex moving boundary problem. Here, beam elements and kinematic sliding joint constraints can be applied to obtain a sound model of the telescopic boom [25].

Multibody dynamics has also proven useful for the design of wind turbines (see Fig. 1d). Here, several purpose built design tools exist 


\section{Flexible multibody dynamics}

for determining the loads acting on a wind turbine e.g. when they are subjected to time series of different varying wind speeds or during a start-up or shut-down sequence. Some of them take advantage of the multibody dynamics framework (e.g. HAWC2 [45]) in order to make coupled models of the wind turbine components, such as the tower, blades and gearbox, and the different control systems. Here, for instance, the incorporation of blade flexibility is crucial to capture the blade deformation as the wind makes the blades deflect and twist. This is important in order to calculate the angle of attack of each blade to calculate the correct lift and drag forces. Multibody dynamics is also used to conduct more detailed studies e.g. on the components in the drive train. One area of interest has been the influence of misalignment between the main shaft and the planetary gearbox on fatigue loads [30]. Here it was concluded that it was necessary to model the bodies as flexible for better comparison with measured data.

The examples listed above all rely on sound and valid multibody dynamics models where flexibility is accounted for in an appropriate manner. The chosen method for modeling the flexibility must be able to accurately represent the deformation that the body undergoes without over-complicating the problem. This could lead to cumbersome modeling or an unnecessarily high number of degrees of freedom (d.o.f.) which will increase the overall analysis time. Depending on the nature of the deformations, several techniques can be applied to describe the flexibility $[74,97]$. The most widely used method is known as the floating frame of reference formulation (FFRF) [93, 13, 85]. In the FFRF, the displacement of a deformable body is divided into large overall motions of a local reference frame fixed to the body, and deformations expressed with respect to this frame. Depending on the shape of the body, the local body deformations can be calculated in several ways $[75,74,70]$.

One group of methods for describing the local body deformations that, in particular, has gained much popularity is the wide selection of component mode synthesis (CMS) based methods. Here, especially, the Craig-Bampton method [15] is one of the most widely used [14]. Overall, when using a CMS-method, it is assumed that the deformation of a body is described as a superposition of a set of pre-calculated mode shapes. The deformation of the body is then calculated as the product of the mode shapes and a set of modal coordinates where each 
mode shape will have a corresponding modal coordinate. The modal coordinates can be interpreted as a set of scale factors, and these are the unknowns that will be calculated during simulation. When using the Craig-Bampton method, the collection of mode shapes consists of both dynamic and static modes. This makes the modal basis capable of describing deformations both due to static as well as dynamic loads.

The use of the FFRF for describing flexible bodies in the multibody dynamics framework has both advantages and disadvantages. The main advantage is that, in general, the stiffness description is simple and linear, which means that stiffness matrices and other necessary data structures associated to calculating the deformations can be defined and calculated in a pre-processing step before simulation. This reduces the computational effort during simulation, but it applies only when small strains and linear material behavior is assumed. The inertia description, on the other hand, becomes cumbersome and highly non-linear due to the fact that deformations are described with respect to a local reference frame that undergoes large rotations. Overall, the FFRF is widely recognized as the standard method for incorporating flexibility in multibody dynamics models due to its good performance, and it has proven its worth in numerous applications.

In some applications, assumptions regarding small strains and linear material behavior can become invalid e.g. due to the loading or the characteristics of the deformable body. For such applications, the FFRF cannot be applied directly. However, studies have been conducted where the FFRF has been applied in large deformation problems, either by using sub-structuring of the flexible body into several interconnected flexible bodies [37] or by including higher order strain terms such that large deformations can be accounted for [57]. Such use of the FFRF complicates the modeling of the flexible body considerably, and makes the stiffness description dependent on the deformed state of the flexible body.

In order to handle problems with large deformations in a more elegant manner, other approaches that are inherently capable of treating combined large deformation and rotation problems have been developed. One is the large rotation vector formulation (LRVF) [83, 84, 97] that is based in the finite element community and has been under development since the middle of the 1980s. In this approach, all state variables are expressed in a global inertial reference frame and the kinematics 
are expressed using both finite displacements and rotations as well as a large strain description. This leads to a finite element formulation capable of describing both large rotations and large deformations. However, the use of both position and rotation d.o.f. in the kinematic description leads to a separate interpolation of these. Besides a more cumbersome implementation due to the separate position and rotation field, the LRVF has also been reported to have a redundant description due to the use of two separate meshes [16], one for the positions and one for the rotations, respectively, which could lead to some challenges when analyzing large deformation problems.

As an alternative to the large rotation vector formulation, the absolute nodal coordinate formulation (ANCF) has been introduced [72]. The ANCF is based in the multibody dynamics community and uses a more consistent kinematic description based on nodal positions and slope vectors defined with respect to a fixed global reference frame. The ANCF has certain advantages when it comes to implementation in the multibody dynamics framework [29]. Due to this, the method has gained much attention in the multibody dynamics research community. However, the ANCF is not perfect and suffers from certain drawbacks, e.g. slow convergence compared to similar methods [6]. The remainder of this thesis is devoted to further investigate some of these drawbacks and their possible remedies.

\section{Absolute nodal coordinate formulation}

The Absolute Nodal Coordinate Formulation (ANCF) was introduced by Shabana $[72,73]$ and since, many researchers have joined to further develop and enhance the method. The ANCF is a non-incremental finite element formulation capable of describing large overall displacements in a straight forward manner. This is accomplished by applying two concepts that, when combined, are unique for the ANCF:

- Nodal positions are described using position vectors expressed in the global inertial reference frame.

- Nodal orientations are described using slope vectors of the position field. 
Using these concepts, a set of element generalized coordinates consisting of nodal positions and slope vectors is chosen. The position field spanned by the nodes is described by interpolation of the nodal positions and slopes ${ }^{1}$ by appropriate interpolation functions. There are several benefits associated to the use of global positions and slope vectors when describing the element kinematics. The most significant being the use of slope vectors to define nodal orientations. Since a vector can be oriented arbitrarily, no assumptions are made regarding the magnitude of nodal rotations. This means that ANCF elements can exhibit arbitrary large rotations. Furthermore, these slope vectors do not have to be perpendicular to each other or be unit vectors. This means that the slope vectors can be used to describe deformation and rotation of the cross section, which increases the modeling fidelity [86]. Additionally, the use of global parameters as generalized coordinates leads to a constant mass matrix and zero centrifugal and Coriolis forces [79]. This simple inertia description makes ANCF attractive for implementation in the multibody dynamics framework.

The use of global position and slope vectors as generalized coordinates is not without problems. Because the element kinematics is based on positions, element strains must be calculated in a way such that rigid body motion produces zero strain. Furthermore, elements that have initial curvature or are skew in their reference configuration must be modeled with care to remove any initial strain energy that could influence simulation results. In general, the stiffness description of ANCF elements is quite cumbersome and several different techniques have been applied to derive the internal elastic forces. Techniques ranging from simple structural mechanics [28] to advanced beam theories [55] or full continuum mechanics [86].

So far several types of different ANCF based finite elements of both beam and shell types have been proposed. The most extensive research has been done within beam type elements. Here several different combinations of generalized coordinates and stiffness descriptions have been investigated. These extensive studies have led to reports and treatment of severe problems with different locking mechanisms [26]. Because of the intensive work of enhancing the ANCF beam el-

\footnotetext{
${ }^{1}$ Throughout this thesis, a slope refers to the gradient of the position field with respect to a local element coordinate
} 
ements, these have been applied in various real life problems such as tire modeling [89], leaf spring modeling [63], catenary systems [46, 71], belt drives [42], and, underground cable installation [99].

Despite the numerous studies within beam elements, ANCF shell elements seems to be lacking the same interest. To this date, only a few different ANCF shell elements can be found in the literature. They are thick fully parameterized rectangular elements [78, 53, 50], thin rectangular elements $[19,21]$, and triangular elements $[18,54]$. In order to take the ANCF shell elements to the same level as the ANCF beam elements, it is evident that the ANCF shell elements need more research attention. Emphasis in this thesis is on rectangular ANCF shell elements. It should be noted, though, that a wide selection of triangular shell elements exist, but these are not treated here.

\section{Scope of work}

The remainder of this thesis is divided into three main parts. The first two parts are primarily the contents of already published work. However, further findings and discussions that are not included in the original papers are presented here. This is done to further emphasize and demonstrate findings and to support conclusions. Part III describes some ongoing and pending work. Thus this part contains some initial thoughts and findings.

Part I is devoted to review and discuss available ANCF shell formulations. Initially, a review of the current state of the art rectangular ANCF shells is given. This includes a novel ANCF shell finite element with dynamic mesh properties introduced during this $\mathrm{PhD}$ project and published in a journal paper [38].

In Part II, the overall behavior of thin rectangular ANCF shell elements is investigated. This includes thorough studies on sensitivity of thin ANCF shell elements with respect to irregular and arbitrary mesh as well as their ability to analyze curved structures. This investigation is motivated by own findings that indicate that results are affected when non-rectangular elements and arbitrary shaped meshes are used. These findings are also published in a journal paper [40]. Towards the end of part II, a modified kinematic description of the thin ANCF shell elements is presented. This modification is intended 
to remedy some of the problems noted in the first sections in Part II. This method is published in a conference paper [39]

In part III, the attention is turned to a new class of ANCF shell elements that has been introduced recently. This element class applies to a wider range of applications since it can express shear and thickness deformations. This means that these elements can be applied for analyzing thick shell structures. Initially, current status with this element class is summarized, and then a new quadratic shear and thickness deformable ANCF shell element is introduced. Here, emphasis is on the description of the internal elastic forces and remedies for alleviating problems regarding locking. The work on the quadratic element is initiated during this $\mathrm{PhD}$ project, but is still in progress.

Finally, the work carried out during the PhD project is discussed and summed up in the closing. Here, possibilities for further work are also mentioned. 


\section{Part I}

\section{Shell elements based on the ANCF}





\section{Shell elements based on the ANCF}

A shell structure is a three dimensional solid structure where one of the dimensions is significantly smaller than the other two. This shorter dimension is referred to as the thickness. When analyzing such a structure using any given finite element method, the overall structure is discretized into smaller shell-like elements for which a set of equilibrium equations can be derived in a systematic manner. Then, the equations for each element are assembled into an overall system of equations that governs the behavior of the structure when subjected to loads and boundary conditions. In this study, the reviewed ANCF elements for analyzing shell and plate structures are all referred to as ANCF shell finite elements even though, they may not be fully generalized to or capable of analyzing arbitrary curved structures. However, it is believed that they can be categorized as shell elements as they can carry both membrane, bending and, for some, transverse shear deformation.

A general three dimensional rectangular ANCF shell finite element is shown in Fig. 2. The position of an arbitrary point $p$ located in the element volume is described by the position vector $\mathbf{r}_{p}$ expressed with respect to the global inertial reference frame $X Y Z$. The position of an arbitrary point in the element volume can be expressed by interpolation of the nodal generalized coordinates using a set of interpolation shape functions.

$$
\mathbf{r}=\mathbf{N q}
$$

where $\mathbf{N}$ is a matrix containing the interpolation shape functions and $\mathbf{q}$ is the vector of element generalized coordinates. $\mathbf{q}$ contains information regarding the element configuration such as the nodal positions and orientations. The generalized coordinates are all expressed 


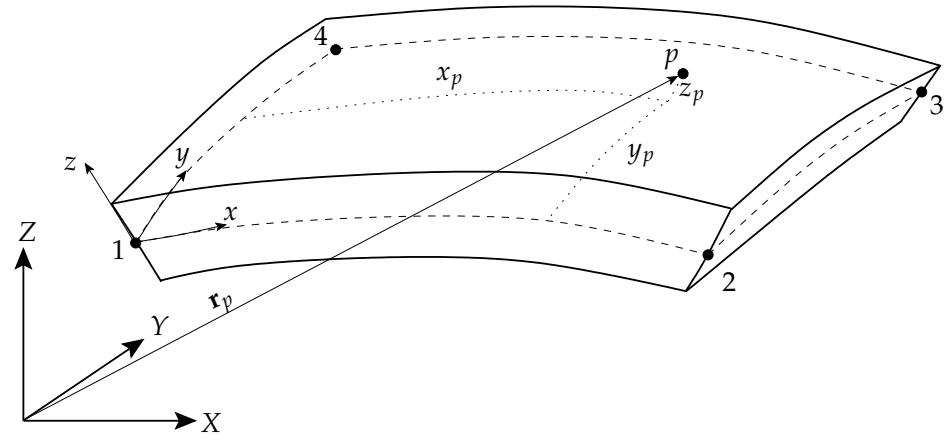

Fig. 2: An ANCF shell element shown with its local element coordinate system. The dashed lines symbolize the element midplane.

with respect to the global reference frame. The interpolation shape functions $\mathbf{N}$ are functions of the local element coordinates $x, y$, and $z$ and can be derived using different approaches depending on the element application and choice of generalized coordinates. The shape functions are constant in time while the generalized coordinates are changing over time.

Using the relationship in Equation (3.1), the position vector $\mathbf{r}_{p}$ (see Fig. 2) is obtained by evaluating $\mathbf{N}$ at the local coordinates $\left(x_{p}, y_{p}, z_{p}\right)$ and multiplying it by the vector of generalized nodal coordinates at the time instance $t_{p}$ corresponding to the element configuration shown in Fig. 2:

$$
\mathbf{r}_{p}=\mathbf{N}\left(x_{p}, y_{p}, z_{p}\right) \mathbf{q}\left(t_{p}\right)
$$

The simple kinematic relation between the nodal coordinates and an arbitrary point in the element volume makes it straightforward to calculate the velocity $\dot{\mathbf{r}}$ and acceleration $\ddot{\mathbf{r}}$ of an arbitrary point as:

$$
\begin{aligned}
& \dot{\mathbf{r}}=\mathbf{N} \dot{\mathbf{q}} \\
& \ddot{\mathbf{r}}=\mathbf{N} \ddot{\mathbf{q}}
\end{aligned}
$$

In the following chapters, two classes of ANCF shell elements will be reviewed. Here the choice of element generalized coordinates will be discussed in depth to clarify which impact the choice of nodal degrees of freedom has on the element application. Furthermore, equilibrium equations for the reviewed ANCF shell elements are derived. 


\section{Fully parameterized shell elements}

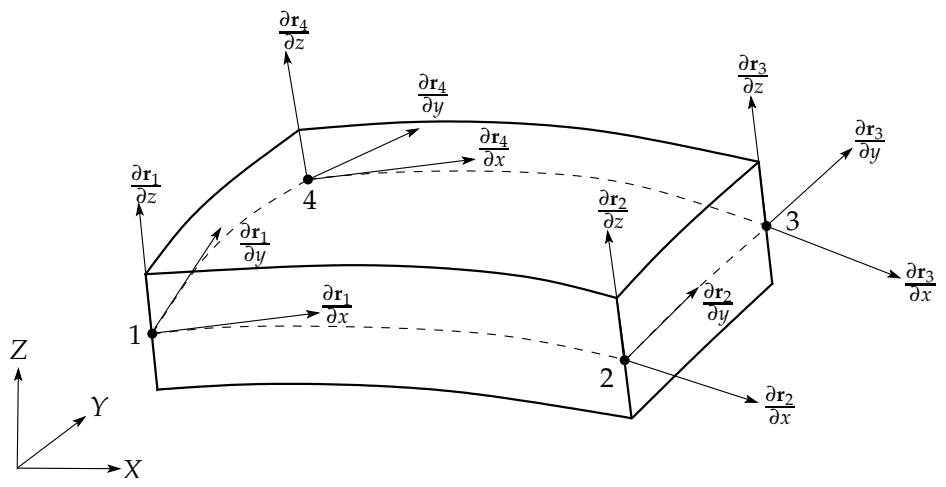

Fig. 3: A fully parameterized ANCF shell element shown with its nodal slope vectors. The dashed lines symbolize the element midplane.

The original ANCF shell element $[53,78]$ is a four noded element that accommodates first order slope vectors with respect to all three local coordinates $x, y$ and $z$ for describing the element kinematics (See Fig. 3). Such an element is often referred to as a fully parameterized element. Together with the position d.o.f., each node, $i$, has twelve d.o.f.:

$$
\mathbf{q}_{i}=\left[\mathbf{r}_{i}^{T}\left(\frac{\partial \mathbf{r}_{i}}{\partial x}\right)^{T}\left(\frac{\partial \mathbf{r}_{i}}{\partial y}\right)^{T}\left(\frac{\partial \mathbf{r}_{i}}{\partial z}\right)^{T}\right]^{T}
$$

Here, $\mathbf{q}_{i}$ is the generalized coordinates for node $i, \mathbf{r}_{i}$ is the position vector of node $i$, and $x, y$ and $z$ are the local element coordinates. This leads to a total of 48 d.o.f. for the entire element.

The use of slope coordinates with respect to all three coordinate axes has certain benefits. First of all, full first order continuity at the nodal points is enforced. This results in a smooth geometrical transition between adjacent elements for both the mid-plane and the cross section. Furthermore, the resulting kinematic description allows for advanced deformation modes. In-plane rotations, i.e. drilling d.o.f., are obtained by the rotation of the in-plane slope vectors, and shear and thickness deformation is available as the transverse slope vector does not need to be perpendicular to the in-plane slope vectors or have a fixed length. Additionally, the large number of d.o.f. leads to 
a high order interpolation which means that a complex shape can be described using a low number of elements.

For this type of element, the stiffness description has been based both on the Kirchhoff theory [78] and a full continuum mechanics approach [53], the latter being the most widely used. The use of the continuum based stiffness description in its pure form is unfavorable as it leads to an overly stiff representation due to locking [50, 51, 69]. Here, it is reported that when the stiffness description is based purely on the interpolated displacement field, the element will suffer from transverse shear, Poisson thickness and curvature locking. The curvature and shear locking can be treated by applying linear interpolation of the shear angles. The Poisson thickness locking is addressed by adding a second order slope vector in the transverse direction which leads to a correct linear description of the transverse normal strain. The extra slope vectors, however, bring the total number of d.o.f. up to 60 d.o.f for the element.

\section{Gradient deficient shell elements}

Soon after the introduction of the fully parameterized shell element, new elements developed specifically for handling thin shell and plate problems were introduced $[19,21]$. Here, two different element parameterizations have been proposed. Common to both of them, however, is that they omit the transverse slope vectors, $\partial \mathbf{r}_{i} / \partial z$, as generalized nodal coordinates (See Fig. 4). This leads to a kinematic description where the shell structure is expressed entirely by the motion and deformation of the shell mid-plane, and, as a consequence, transverse shear and thickness deformation cannot be described. As this element type does not employ a full set of slope coordinates, the element is often referred to as a gradient deficient element. In this study, the gradient deficient shell element is referred to as a thin ANCF shell element.

The original thin ANCF shell element [19] uses nodal positions, two first order, and one second order slope vector as generalized nodal coordinates:

$$
\mathbf{q}_{i}=\left[\mathbf{r}_{i}^{T}\left(\frac{\partial \mathbf{r}_{i}}{\partial x}\right)^{T}\left(\frac{\partial \mathbf{r}_{i}}{\partial y}\right)^{T}\left(\frac{\partial^{2} \mathbf{r}_{i}}{\partial x \partial y}\right)^{T}\right]^{T}
$$


5. Gradient deficient shell elements

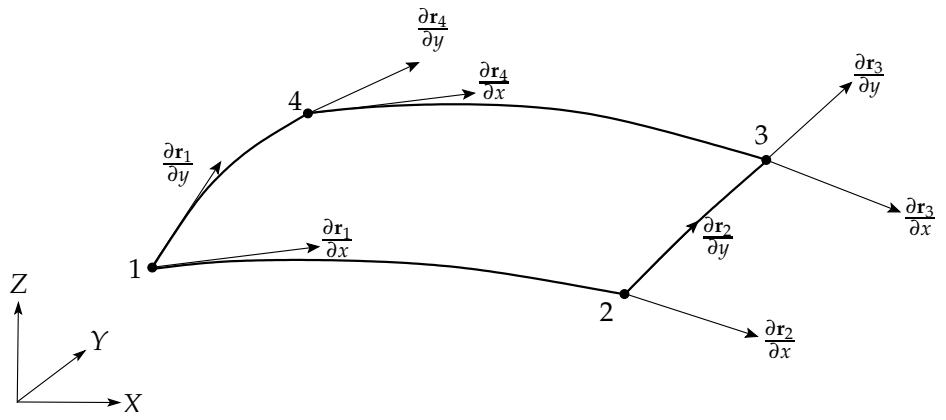

Fig. 4: A gradient deficient thin ANCF shell element shown with its nodal slope vectors.

This leads to an element with 48 d.o.f. The two first order slope vectors are used to describe in-plane deformation and nodal orientation while the second order slope vector is associated with the curvature of the element. The element kinematics is described using a set of shape functions based products of Hermite beam shape functions [101]. Using this approach, $C^{1}$ continuous interpolation is ensured. The stiffness description is based on the Kirchoff plate theory, and numerous comparisons between numerical examples and corresponding analytical results are presented with good agreement.

The authors of the original thin ANCF shell element [19] also mention the possibility of constructing a simplified element with only 36 d.o.f. This should be done by omitting the second order slope vector from the generalized coordinates as follows:

$$
\mathbf{q}_{i}=\left[\mathbf{r}_{i}^{T}\left(\frac{\partial \mathbf{r}_{i}}{\partial x}\right)^{T}\left(\frac{\partial \mathbf{r}_{i}}{\partial y}\right)^{T}\right]^{T}
$$

Such an element has been implemented and studied in detail [21]. In this study, the kinematic description utilizes shape functions derived using an incomplete quartic polynomial. Using this polynomial expansion, only $C^{0}$ continuity between adjacent elements can be ensured. The stiffness description is also based on the Kirchhoff shell theory, and numerical results for thin plate problems are compared to results obtained using the fully parameterized element [53]. The comparison shows that the thin ANCF shell element shows significantly better convergence and faster computational speed when compared to the 
fully parameterized element when thin shell structures are analyzed. This observation also supports the observations regarding locking in the fully parameterized ANCF shell element.

\section{Equations of motion}

In this section, the governing equations for ANCF shell elements are derived. That being the equations of motion, since the ANCF formulation is intended for implementation in the multibody dynamics framework. The equations are derived in a general manner and two approaches for calculating the internal elastic forces are described. At the end, a numerical procedure for obtaining static equilibrium is given.

The equations of motion for an ANCF element can be derived using several techniques, e.g. using the D'Alembert-Lagrange equations or Hamilton's principle [75]. Another way is to calculate the virtual work $\delta W$ for a system in dynamic equilibrium:

$$
\delta W=\delta W_{i}+\delta W_{e}-\delta W_{f}=0
$$

Here $\delta W_{i}, \delta W_{e}$, and $\delta W_{f}$ denote the virtual work done by inertia, internal elastic, and external forces, respectively. The virtual work done by the inertia and external forces can be calculated as follows.

$$
\begin{aligned}
& \delta W_{i}=\int_{V_{0}} \delta \mathbf{r}^{T} \ddot{\mathbf{r}} \rho \mathrm{d} V \\
& \delta W_{f}=\int_{V_{0}} \delta \mathbf{r}^{T} \mathbf{F d} V
\end{aligned}
$$

where $\mathbf{F}$ is the total externally applied force vector and $\rho$ is the material density. Note that the integration is done over the volume at the reference configuration $V_{0}$. The virtual displacement $\delta \mathbf{r}$ is expressed as:

$$
\delta \mathbf{r}=\mathbf{N} \delta \mathbf{q}
$$

By combination of Equations (3.3), (6.8), and (6.9), expressions for the element mass matrix and the generalized external forces are obtained:

$$
\begin{aligned}
\delta W_{i} & =\delta \mathbf{q}^{T} \rho \int_{V_{0}} \mathbf{N}^{T} \mathbf{N} \mathrm{d} V \ddot{\mathbf{r}}=\delta \mathbf{q}^{T} \mathbf{M} \ddot{\mathbf{q}} \\
\delta W_{f} & =\delta \mathbf{q}^{T} \int_{V_{0}} \mathbf{N}^{T} \mathbf{F d} V=\delta \mathbf{q}^{T} \mathbf{Q}_{f}
\end{aligned}
$$


where $\mathbf{M}$ is the element mass matrix and $\mathbf{Q}_{f}$ is the generalized external forces. The virtual work done by the internal elastic forces is calculated as [77]:

$$
\delta W_{e}=\delta \mathbf{q}^{T} \frac{\partial W_{e}}{\partial \mathbf{q}}=\delta \mathbf{q}^{T} \mathbf{Q}_{e}
$$

where $W_{e}$ is an expression for the internal elastic energy and $\mathbf{Q}_{e}$ is the vector of internal elastic forces. This expression is general and the elastic energy can be calculated in various ways depending on the element type and application. Examples of this is given in Section 6.1.

By combining Equations (6.10) and (6.11) and utilizing that the resulting equation must hold true for any arbitrary virtual displacement [31], the equations of motion take the form:

$$
\mathbf{M} \ddot{\mathbf{q}}=\mathbf{Q}_{f}-\mathbf{Q}_{e}
$$

where $\mathbf{M}$ and $\mathbf{Q}_{f}$ are given by:

$$
\begin{aligned}
\mathbf{M} & =\rho \int_{V_{0}} \mathbf{N}^{T} \mathbf{N d} V \\
\mathbf{Q}_{f} & =\int_{V_{0}} \mathbf{N}^{T} \mathbf{F d} V
\end{aligned}
$$

As seen, the resulting equations are very straightforward, which is mainly due to the simple kinematic description that is based on interpolation of global position and slope vectors (see Equation (3.1)). The constant mass matrix and absence of centrifugal and Coriolis forces are other consequences of this.

The equations of motion for ANCF elements, as stated in Equation (6.12), has a form that makes it very suitable for combining with the Newton-Euler equations that govern rigid multibody systems [31]. When combined, the full equation set takes the form:

$$
\left[\begin{array}{cccc}
\mathbf{M}^{(\mathbf{R})} & 0 & 0 & \left(\frac{\partial \Phi}{\partial \mathbf{R}}\right)^{T} \\
0 & \mathbf{J}^{\prime} & 0 & \left(\frac{\partial \Phi}{\partial \theta}\right)^{T} \\
0 & 0 & \mathbf{M}^{(\mathbf{q})} & \left(\frac{\partial \Phi}{\partial \mathbf{q}}\right)^{T} \\
\left(\frac{\partial \Phi}{\partial \mathbf{R}}\right) & \left(\frac{\partial \Phi}{\partial \theta}\right) & \left(\frac{\partial \Phi}{\partial \mathbf{q}}\right) & 0
\end{array}\right]\left\{\begin{array}{c}
\ddot{\mathbf{R}} \\
\dot{\omega}^{\prime} \\
\ddot{\mathbf{q}} \\
\lambda
\end{array}\right\}=\left\{\begin{array}{c}
\mathbf{Q}_{f}^{(\mathbf{R})} \\
\mathbf{m}^{\prime}-\tilde{\boldsymbol{\omega}}^{\prime} \mathbf{J}^{\prime} \boldsymbol{\omega}^{\prime} \\
\mathbf{Q}_{f}^{(\mathbf{q})}-\mathbf{Q}_{e} \\
\mathbf{Q}_{d}
\end{array}\right\}
$$


Here, $\mathbf{R}$ is the vector of rigid body translational coordinates and $\boldsymbol{\theta}$ is the vector of the rigid body rotational parameters. $\omega^{\prime}$ is the vector of the local angular velocities, $\mathbf{m}^{\prime}$ is the vector of applied moments and $\mathbf{J}^{\prime}$ is the inertia matrix, all associated with the rigid bodies. In Equation (6.14), the Lagrange multiplier theorem has been applied to enforce constraints with $\boldsymbol{\Phi}$ being the vector of constraint equations and $\lambda$ are the Lagrange multipliers associated with the acting constraint equations. The superscripts associated with $\mathbf{M}$ and $\mathbf{Q}_{f}$ refer to their associated coordinates. $\mathbf{Q}_{d}$ is an additional force term arising due to the fact that the constraints are enforced on acceleration level [76]. Using this equation set, coupled rigid body and ANCF multibody systems can be analyzed.

\subsection{Calculation of internal elastic forces}

The calculation of the internal elastic forces arising in the ANCF elements due to deformation is a challenging aspect of the ANCF and has been subject to much research. In general, two different approaches can be applied. The first approach is based on treating the ANCF element as a solid volume and using continuum mechanics for calculating the internal stresses and strains. This approach was used in the original fully parameterized element [53]. The second approach is based on structural mechanics, where the ANCF element is treated like a structural element and the internal elastic energy is calculated using appropriate shell theories.

\section{Continuum mechanics approach}

When using the continuum mechanics (CM) approach, special care must be taken when selecting the procedure for calculating the strains. Because the ANCF elements are defined using absolute positions, the calculation of strains must be based on the position gradients. Methods based on displacement gradients can be applied, but that would require the displacements to have to been extracted separately, which would further complicate the calculation of the elastic forces. Furthermore, it is crucial that the strain measure produces zero strain in the case of pure rigid body motion.

The strains in an ANCF element are calculated using the Green- 
6. Equations of motion

Lagrange strain tensor E given by [43]:

$$
\mathbf{E}=\frac{1}{2}\left(\mathbf{F}^{T} \mathbf{F}-\mathbf{I}\right)
$$

where $\mathbf{F}$ is the deformation gradient tensor and $\mathbf{I}$ is the identity matrix. The deformation gradient tensor is defined as:

$$
\mathbf{F}=\frac{\partial \mathbf{r}}{\partial \mathbf{r}_{0}}=\left(\frac{\partial \mathbf{r}}{\partial \mathbf{x}}\right)\left(\frac{\partial \mathbf{r}_{0}}{\partial \mathbf{x}}\right)^{-1}=\mathbf{J}\left(\mathbf{J}_{0}\right)^{-1}
$$

Here, $\mathbf{r}_{0}$ is the position vector field of the element in the initial reference configuration and $\mathbf{x}$ is the full set of local element coordinates, hence $\mathbf{x}=\left[\begin{array}{ll}x & y \\ z\end{array}\right]^{T}$. This is very convenient since the $\mathbf{J}$ matrix is simply constructed by ordering the three slope vectors in the current state $\partial \mathbf{r} / \partial x, \partial \mathbf{r} / \partial y$ and $\partial \mathbf{r} / \partial z$ together in a $3 \times 3$ matrix. The same goes for the $\mathbf{J}_{0}$ matrix. However here the slope vectors are evaluated at the initial reference state. In the case where an initially straight element is analyzed, $\mathbf{J}_{0}$ will be the identity matrix. If the element is curved, skew or 'deformed' in other ways initially, $\mathbf{J}_{0}$ will ensure that the calculated strains are calculated with respect to the reference state and that the element is strain-free in the initial state.

Based on the Green-Lagrange strain tensor, as defined in Equation (6.15), the internal elastic energy is calculated as:

$$
W_{e}=\int_{V_{0}} \mathbf{E}: \mathbf{S} \mathrm{d} V
$$

which can be inserted into Equation (6.11) to obtain an expression for the internal elastic force vector. In Equation (6.17), : denotes the double dot product and $\mathbf{S}$ is the second Piola-Kirchhoff stress tensor. Here, the second Piola-Kirchhoff stress tensor is used as it is work conjugated with the Green-Lagrange strain tensor [43].

\section{Structural mechanics approach}

As an alternative to the CM approach, the internal elastic forces can be calculated using a structural mechanics (SM) approach. Here, the internal elastic energy is calculated used well-known shell theory. In this study, the SM approach is used to calculate the elastic force vector for the gradient deficient thin ANCF shell elements. As the element is 
not capable of describing transverse shear deformation, the Kirchhoff shell theory is utilized [91]. Here, the elastic energy is segregated into two parts. One part consists of the energy arising due to membrane stretch and shear while the second part accounts for the energy arising due to bending and twisting of the midplane. The internal elastic energy is expressed as:

$$
W_{e}=\frac{h}{2} \int_{A_{0}} \varepsilon^{T} \mathbf{D}_{m} \varepsilon \mathrm{d} A+\frac{1}{2} \int_{A_{0}} \boldsymbol{\kappa}^{T} \mathbf{D}_{\kappa} \boldsymbol{\kappa} \mathrm{d} A,
$$

where $h$ is the element thickness, $A_{0}$ is the element area in the initial configuration, $\varepsilon$ is a vector containing the in-plane normal and shear strains and $\kappa$ is a vector containing midplane curvatures. The matrices $\mathbf{D}_{m}$ and $\mathbf{D}_{\kappa}$ couple the membrane and curvature strains with their respective stresses and are given by:

$$
\begin{aligned}
& \mathbf{D}_{m}=\frac{E}{1-v^{2}}\left[\begin{array}{ccc}
1 & v & 0 \\
v & 1 & 0 \\
0 & 0 & \frac{1-v}{2}
\end{array}\right] \\
& \mathbf{D}_{\kappa}=\frac{h^{3}}{12} \mathbf{D}_{m}
\end{aligned}
$$

Here, $E$ is the Young's modulus and $v$ is the Poisson's ratio. The matrix $\mathbf{D}_{m}$ is the two dimensional constitutive relation for plane stress condition [14]. Here, it is assumed that the material is linear elastic and isotropic. The matrix $\mathbf{D}_{\kappa}$ arises as the integration over the thickness in the last term in Equation (6.18), containing the energy associated to the element curvatures, can be carried out analytically.

The in-plane strains for an initially straight and rectangular element with the local element coordinate system aligned with the global inertial reference frame are calculated using the general Green-Lagrange strain tensor in Voigt notation:

$$
\begin{gathered}
\varepsilon=\left[\begin{array}{lll}
\varepsilon_{x x} & \varepsilon_{y y} & \gamma_{x y}
\end{array}\right]^{T} \\
\varepsilon_{x x}=\frac{\left(\frac{\partial \mathbf{r}}{\partial x}\right)^{T}\left(\frac{\partial \mathbf{r}}{\partial x}\right)-1}{2}, \quad \varepsilon_{y y}=\frac{\left(\frac{\partial \mathbf{r}}{\partial y}\right)^{T}\left(\frac{\partial \mathbf{r}}{\partial y}\right)-1}{2}, \\
\gamma_{x y}=\left(\frac{\partial \mathbf{r}}{\partial x}\right)^{T}\left(\frac{\partial \mathbf{r}}{\partial y}\right)
\end{gathered}
$$


The calculation of the curvatures $\kappa$ of ANCF elements has been subject to much discussion and several approaches have been suggested [19, 27, 29]. In this study, the curvature of the thin ANCF shell elements is calculated as suggested in the original papers regarding thin ANCF shell elements [19, 21]:

$$
\begin{gathered}
\boldsymbol{\kappa}=\left[\begin{array}{lll}
\kappa_{x x} & \kappa_{y y} & 2 \kappa_{x y}
\end{array}\right]^{T} \\
\kappa_{x x}=\frac{\left(\frac{\partial^{2} \mathbf{r}}{\partial x^{2}}\right)^{T} \mathbf{n}}{\|\mathbf{n}\|^{3}}, \quad \kappa_{y y}=\frac{\left(\frac{\partial^{2} \mathbf{r}}{\partial y^{2}}\right)^{T} \mathbf{n}}{\|\mathbf{n}\|^{3}}, \\
\kappa_{x y}=\frac{\left(\frac{\partial^{2} \mathbf{r}}{\partial x \partial y}\right)^{T} \mathbf{n}}{\|\mathbf{n}\|^{3}}
\end{gathered}
$$

The mid-surface normal vector $\mathbf{n}$ is defined as $\mathbf{n}=\frac{\partial \mathbf{r}}{\partial x} \times \frac{\partial \mathbf{r}}{\partial y}$.

\subsection{Static equilibrium}

Static solutions are obtained by calculating a set of nodal coordinates $\mathbf{q}$ yielding static equilibrium between the externally applied force $\mathbf{Q}_{f}$ and the internal elastic forces $\mathbf{Q}_{e}$. The set of nodal coordinates is calculated using an iterative solution technique [86]:

$$
\mathbf{q}^{(n+1)}=\mathbf{q}^{(n)}-\left(\mathbf{K}_{T}^{(n)}\right)^{-1}\left(\mathbf{Q}_{e}^{(n)}-\mathbf{Q}_{f}^{(n)}\right) .
$$

In Equation (6.22), $\mathbf{q}^{(n+1)}$ is the updated solution, $\mathbf{q}^{(n)}$ is the current solution, $\mathbf{K}_{T}^{(n)}$ is the tangent stiffness matrix at the current iteration step and $\mathbf{Q}_{e}^{(n)}$ and $\mathbf{Q}_{f}^{(n)}$ are the elastic and external force vectors, respectively, at the current iteration step. The iteration loop is terminated when the norm of the residual between $\mathbf{Q}_{e}^{(n)}$ and $\mathbf{Q}_{f}^{(n)}$ is below predefined tolerance. The tangent stiffness matrix $\mathbf{K}_{T}^{(n)}$ is a highly nonlinear function of the nodal coordinates and is calculated as:

$$
\mathbf{K}_{T}^{(n)}=\frac{\partial \mathbf{Q}_{e}^{(n)}}{\partial \mathbf{q}^{(n)}} .
$$

In this investigation, the tangent stiffness matrix is evaluated numerically using positive perturbation of the nodal coordinates and finite differences. 


\subsection{Discussion}

The derivation of the equations of motion has clarified some of the advantages and drawbacks associated with the ANCF. First and foremost, the constant mass matrix and the absence of centrifugal and Coriolis forces are highly notable. This is a consequence of using generalized coordinates expressed in a global reference frame. Because the mass matrix remains constant over time, it can be evaluated in a pre-processing step before the actual simulation forward in time is performed. Furthermore, the integral in the expression for the mass matrix can, in general, be solved analytically. The same goes for the expression of the external forces. This ensures fast numerical evaluation. The simple expressions for the mass matrix and external forces, together with the simple kinematic description, are the key benefits that make the ANCF attractive for implementation in the multibody dynamics framework.

Two different approaches for calculating the internal elastic forces have been reviewed. The CM based approach for calculating the internal elastic forces leads to a general and very versatile description. Here, any given constitutive relation can be applied since no assumptions are introduced regarding the calculation of the stresses $\mathbf{S}$. This means that a wide variety of material behavior including plasticity and yielding $[87,88]$ can be described. However, in order to apply the $\mathrm{CM}$ approach for deriving the elastic forces, a full set of local element coordinates must be present. This means that it only applies to fully parameterized elements or similar. As a consequence, the approach does not apply for the thin ANCF shell element. Furthermore, the use of the $\mathrm{CM}$ approach increases the computational effort since the numerical integration of Equation (6.17) must be carried out as a volume integration. When using the SM approach, the numerical integration of the internal elastic energy (Equation (6.18)) is modified such that integration over the element area is sufficient. This reduces the number of necessary sampling points, which leads to reduced computational effort.

As mentioned in the introduction, the ANCF is not without difficulties. These difficulties are mainly tied to the vector of elastic forces. In general, the elastic forces are highly non-linear functions of the generalized coordinates. Even when the elastic forces are based on simple 
expressions from structural mechanics. In this case where the Kirchhoff plate theory is applied it is the expressions for the element curvature in particular that are highly non-linear (see Equation (6.21)). The calculation of the element curvature is also a point of concern. Many approaches have been suggested and some even state that the approach which has been adopted here (see Equation (6.21)) is incorrect and should be avoided [27]. However, in this thesis, the thin ANCF shell elements will only be subjected to comparative studies where the different formulations are compared. In these investigations, the different formulations will all use the same procedure for calculation of the elastic forces, hence the comparison will be made on common conditions. However, it could be interesting to do a thorough investigation on how the calculation of the element curvature affects simulation results. Such an investigation could help future users in choosing the best method for their specific application and act as a guideline in the use of ANCF shell elements that applies the Kirchhoff shell theory in calculation of the elastic forces.

\section{A combined ALE-ANCF shell element}

This chapter contains a review of the shell element introduced by Hyldahl et al. [38]. In this paper, a novel shell finite element based on the combined arbitrary Lagrange-Euler and absolute nodal coordinate formulations (ALE-ANCF) is introduced. This element is distinguished by applying extra nodal d.o.f. to describe the relative nodal positions in the mesh. This allow the nodes to travel in the modeled specimen, making the element particularly suited for modeling varying boundary conditions such as sliding joints and traveling forces. Finally, further discussion regarding the obtained results and possible applications that have not been included in the original journal paper is given at the end of the section.

\subsection{Kinematics of ALE-ANCF based shell elements}

The combination of the arbitrary Lagrange-Euler and the absolute nodal coordinate formulation was introduced to analyze large displacements in very elastic specimens with internal mass flow and sliding joints $[35,34]$. In these studies, focus is on specimens that can 
be treated as one-dimensional. This leads to a structural description based on well-known beam theory. The innovation associated with the study is the kinematic description. Here, the authors are inspired by the mobile element kinematics known from the Arbitrary LagrangeEuler (ALE) description [20]. In the ALE description, the nodal positions are not fixed either to the modeled specimen or to the global reference frame. Instead, the nodes are allowed to move arbitrarily, which can be of great advantage in cases where either the domain or the specimen undergo large deformation. Therefore, the ALE mesh description is widely used e.g. in computational fluid dynamics (CFD) or non-linear solid mechanics. A good example where the ALE description is advantageous is in the simulation of forming processes of metals. Here, significant re-meshing can be avoided when applying the ALE method [68].

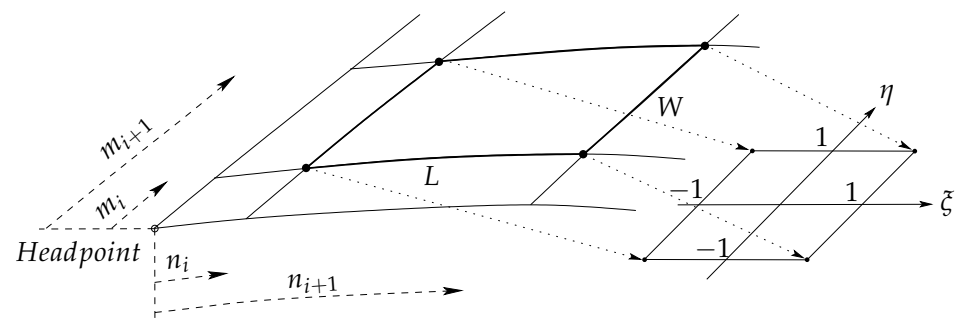

Fig. 5: Plate element in two-dimensional mesh mapped into isoparametric coordinates.

The idea of the moving nodes is transfered to the ANCF to formulate the kinematics of an ANCF shell element with non-stationary nodes. The ALE-ANCF shell element is based on a 36 d.o.f. thin ANCF shell element (See Fig. 4 and Equation (5.6)). An additional set of generalized coordinates $n_{i}$ and $m_{i}$ that describes the nodal position in the modeled specimen is introduced (see Fig. 5). The position of the nodes is measured with respect to a fixed reference point called the head point. For convenience, the head point is located at a corner of the modeled specimen. Combining the extra set of local material coordinates with the general ANCF coordinates, see Equation (5.6), results in 
an expression for the total vector of element generalized coordinates.

$$
\begin{aligned}
& \mathbf{q}_{e}=\left[\mathbf{r}_{1}^{T}\left(\frac{\partial \mathbf{r}_{1}}{\partial x}\right)^{T}\left(\frac{\partial \mathbf{r}_{1}}{\partial y}\right)^{T} \ldots \mathbf{r}_{4}^{T}\left(\frac{\partial \mathbf{r}_{4}}{\partial x}\right)^{T}\left(\frac{\partial \mathbf{r}_{4}}{\partial y}\right)^{T}\right]^{T} \\
& \mathbf{q}_{l}=\left[\begin{array}{llll}
n_{1} & m_{1} & n_{2} & m_{2}
\end{array}\right]^{T} \\
& \mathbf{q}=\left[\begin{array}{ll}
\mathbf{q}_{e}^{T} & \mathbf{q}_{l}^{T}
\end{array}\right]^{T}
\end{aligned}
$$

where $\mathbf{q}_{e}$ is the vector of ANCF coordinates, $\mathbf{q}_{l}$ contains the local material coordinates, and $\mathbf{q}$ is the full vector of generalized coordinates. By the introduction of the coordinates $n_{i}$ and $m_{i}$, each element is allowed to change its size dynamically over time. This means that the isoparametric mapping of each element becomes unique and time dependent.

The varying isoparametric mapping is accounted for by expressing the isoparametric mapping parameters $\xi$ and $\eta$ in terms of the local material coordinates $n_{i}$ and $m_{i}$.

$$
\begin{array}{ll}
\xi=\frac{2 n-n_{1}-n_{2}}{n_{2}-n_{1}}, & n_{1} \leq n \leq n_{2} \\
\eta=\frac{2 m-m_{1}-m_{2}}{m_{2}-m_{1}}, & m_{1} \leq m \leq m_{2}
\end{array}
$$

Furthermore, the element side lengths can be expressed in terms of $n_{i}$ and $m_{i}$.

$$
\begin{aligned}
& L=n_{2}-n_{1} \\
& W=m_{2}-m_{1}
\end{aligned}
$$

The position vector of an arbitrary point on an element $\mathbf{r}$ is obtained in the normal manner by interpolation of the nodal position and slope vectors.

$$
\mathbf{r}=\mathbf{N}_{e} \mathbf{q}_{e}
$$

where $\mathbf{N}_{e}$ is the $3 \times 36$ interpolation shape function matrix and $\mathbf{q}_{e}$ is the vector of generalized ANCF nodal coordinates. The shape functions for a 36 d.o.f. thin ANCF shell element can be derived using either the crossed beam technique [19] or based on an incomplete quartic polynomial [21]. In this study, shape functions based on the crossed beam technique are chosen. The shape functions are given in the original research paper [38]. 
Because of the time varying isoparametric mapping, the shape functions are functions of the local material coordinates $n_{i}$ and $m_{i}$. This means that the shape functions are time dependent. As a consequence, the chain rule of differentiation must be applied when calculating the velocity and acceleration of an arbitrary point in the element.

$$
\begin{aligned}
& \dot{\mathbf{r}}=\mathbf{N}_{e} \dot{\mathbf{q}}_{e}+\dot{\mathbf{N}}_{e} \mathbf{q}_{e} \\
& \ddot{\mathbf{r}}=\mathbf{N}_{e} \ddot{\mathbf{q}}_{e}+2 \dot{\mathbf{N}}_{e} \dot{\mathbf{q}}_{e}+\ddot{\mathbf{N}}_{e} \mathbf{q}_{e}
\end{aligned}
$$

It can be shown that by rearranging and collecting terms the velocity and acceleration vectors can be written in simpler form:

$$
\begin{aligned}
\dot{\mathbf{r}} & =\hat{\mathbf{N}} \dot{\mathbf{q}} \\
\ddot{\mathbf{r}} & =\hat{\mathbf{N}} \ddot{\mathbf{q}}+\ddot{\mathbf{r}}_{p}
\end{aligned}
$$

The quantities $\hat{\mathbf{N}}$ and $\ddot{\mathbf{r}}_{p}$ take a cumbersome form, and especially $\ddot{\mathbf{r}}_{p}$ is a highly non-linear function of both the nodal coordinates and the nodal velocities. $\hat{\mathbf{N}}$ and $\ddot{\mathbf{r}}_{p}$ are given by:

$$
\begin{aligned}
& \hat{\mathbf{N}}=\left[\begin{array}{ll}
\mathbf{N}_{e} & \mathbf{N}_{q}
\end{array}\right] \\
& \mathbf{N}_{q}=\left[\frac{\partial \mathbf{N}_{e}}{\partial n_{1}} \mathbf{q}_{e} \frac{\partial \mathbf{N}_{e}}{\partial m_{1}} \mathbf{q}_{e} \frac{\partial \mathbf{N}_{e}}{\partial n_{2}} \mathbf{q}_{e} \frac{\partial \mathbf{N}_{e}}{\partial m_{2}} \mathbf{q}_{e}\right]
\end{aligned}
$$

and

$$
\ddot{\mathbf{r}}_{p}=2 \mathbf{N}_{q t} \dot{\mathbf{q}}_{l}+\mathbf{N}_{q q} \dot{\mathbf{q}}_{l}^{2}+2 \mathbf{N}_{t t} \mathbf{q}_{e}
$$

where

$$
\begin{aligned}
\mathbf{N}_{q t}= & {\left[\frac{\partial \mathbf{N}_{e}}{\partial n_{1}} \dot{\mathbf{q}}_{e} \frac{\partial \mathbf{N}_{e}}{\partial m_{1}} \dot{\mathbf{q}}_{e} \frac{\partial \mathbf{N}_{e}}{\partial n_{2}} \dot{\mathbf{q}}_{e} \frac{\partial \mathbf{N}_{e}}{\partial m_{2}} \dot{\mathbf{q}}_{e}\right] } \\
\mathbf{N}_{q q}= & {\left[\frac{\partial^{2} \mathbf{N}_{e}}{\partial n_{1}^{2}} \mathbf{q}_{e} \frac{\partial^{2} \mathbf{N}_{e}}{\partial m_{1}^{2}} \mathbf{q}_{e} \frac{\partial^{2} \mathbf{N}_{e}}{\partial n_{2}^{2}} \mathbf{q}_{e} \frac{\partial^{2} \mathbf{N}_{e}}{\partial m_{2}^{2}} \mathbf{q}_{e}\right] } \\
\mathbf{N}_{t t=}= & \frac{\partial^{2} \mathbf{N}_{e}}{\partial n_{1} \partial m_{1}} \dot{n_{1}} \dot{m}_{1}+\frac{\partial^{2} \mathbf{N}_{e}}{\partial n_{1} \partial n_{2}} \dot{n_{1}} \dot{n_{2}}+\frac{\partial^{2} \mathbf{N}_{e}}{\partial n_{1} \partial m_{2}} \dot{n_{1}} \dot{m_{2}}+ \\
& \frac{\partial^{2} \mathbf{N}_{e}}{\partial m_{1} \partial n_{2}} \dot{m_{1}} \dot{n_{2}}+\frac{\partial^{2} \mathbf{N}_{e}}{\partial m_{1} \partial m_{2}} \dot{m_{1}} \dot{m_{2}}+\frac{\partial^{2} \mathbf{N}_{e}}{\partial n_{2} \partial m_{2}} \dot{m_{2}} \dot{m_{2}}
\end{aligned}
$$




\subsection{Equations of motion}

The equations of motion for the ALE-ANCF shell element are derived as in the traditional ANCF by employing the principle of virtual work for a system in dynamic equilibrium (see Equation (6.7)). In the calculation of the virtual work, the virtual displacement, $\delta \mathbf{r}$, is needed. In the general ANCF, $\delta \mathbf{r}$ can be rewritten in terms of the generalized coordinates in a straightforward manner as $\delta \mathbf{r}=\mathbf{N} \delta \mathbf{q}$. In the ALE-ANCF, however, the chain rule of differentiation must be applied due to the time varying shape functions. The virtual displacement is expressed as:

$$
\delta \mathbf{r}=\mathbf{N}_{e} \delta \mathbf{q}_{e}+\delta \mathbf{N}_{e} \mathbf{q}_{e}=\hat{\mathbf{N}} \delta \mathbf{q}
$$

The virtual work done by the applied and elastic forces is calculated in a straightforward manner as in the general ANCF which is given in Section 6. The virtual work done by the inertia forces $\delta W_{i}$, however, takes a more extensive form due to the extra terms in the acceleration vector (see Equation (7.29)). The virtual work done by the inertia forces is calculated as:

$$
\begin{aligned}
& \delta W_{i}=\rho \int_{V} \delta \mathbf{r}^{T}\left(\hat{\mathbf{N}} \ddot{\mathbf{q}}+\ddot{\mathbf{r}}_{p}\right) \mathrm{d} V \Leftrightarrow \\
& \delta W_{i}=\delta \mathbf{q}^{T}\left(\rho \int_{V} \hat{\mathbf{N}}^{T} \hat{\mathbf{N}} \mathrm{d} V \ddot{\mathbf{q}}+\rho \int_{V} \hat{\mathbf{N}}^{T} \ddot{\mathbf{r}}_{p} \mathrm{~d} V\right)
\end{aligned}
$$

This shows that, in addition to a traditional mass matrix, an additional inertia force vector arises.

The equations of motion are assembled by combining Equation (7.34) with the expressions for the virtual work done by the external and elastic forces. The constraint reaction forces are accounted for by using Lagrange multipliers [31]. Hereby, the equations of motion for an ALE-ANCF shell element take the form:

$$
\mathbf{M} \ddot{\mathbf{q}}=\mathbf{Q}_{f}-\mathbf{Q}_{e}-\mathbf{Q}_{p}-\left(\frac{\partial \boldsymbol{\Phi}}{\partial \mathbf{q}}\right)^{T} \lambda
$$


with

$$
\begin{aligned}
& \mathbf{M}=\rho h \int_{n_{1}}^{n_{2}} \int_{m_{1}}^{m_{2}} \tilde{\mathbf{N}}^{T} \tilde{\mathbf{N}} \mathrm{d} m \mathrm{~d} n \\
& \mathbf{Q}_{f}=\int_{n_{1}}^{n_{2}} \int_{m_{1}}^{m_{2}} \tilde{\mathbf{N}}^{T} \mathbf{F} \mathrm{d} m \mathrm{~d} n \\
& \mathbf{Q}_{e}=h \int_{n_{1}}^{n_{2}} \int_{m_{1}}^{m_{2}}\left(\frac{\partial \boldsymbol{\varepsilon}}{\partial \mathbf{q}}\right)^{T} \mathbf{D}_{m} \varepsilon \mathrm{d} m \mathrm{~d} n+\int_{n_{1}}^{n_{2}} \int_{m_{1}}^{m_{2}}\left(\frac{\partial \boldsymbol{\kappa}}{\partial \mathbf{q}}\right)^{T} \mathbf{D}_{\kappa} \boldsymbol{\kappa} \mathrm{d} m \mathrm{~d} n \\
& \mathbf{Q}_{p}=\rho h \int_{n_{1}}^{n_{2}} \int_{m_{1}}^{m_{2}} \tilde{\mathbf{N}}^{T} \ddot{\mathbf{r}}_{p} \mathrm{~d} m \mathrm{~d} n
\end{aligned}
$$

Here, $\mathbf{M}$ is the element mass matrix, $\mathbf{Q}_{f}$ is the generalized applied force vector, $\mathbf{Q}_{e}$ is the vector of elastic forces, and $\mathbf{Q}_{p}$ is an inertia force vector that depends quadratically on velocities. The constraint reaction forces are calculated as the product of the constraint Jacobian $\frac{\partial \Phi}{\partial \mathbf{q}}$ and the vector of Lagrange multipliers $\lambda$.

$\mathbf{Q}_{p}$ is unique for the ALE-ANCF and arises because the acceleration, as defined in Equation (7.29), is a function of both the generalized coordinates, $\mathbf{q}_{e}$, and generalized velocities, $\dot{\mathbf{q}}_{e}$. The element mass matrix will also vary with time due to shape function time dependency. However, according to Equations (7.31) and (7.32), if the local material coordinates $m_{i}$ and $n_{1}$ are kept fixed, then $\dot{n}_{i}=\dot{m}_{i}=0$, and $\mathbf{Q}_{p}$ will vanish. The mass matrix, on the other hand, will continue to vary with time because the four last terms in $\tilde{\mathbf{N}}$ are functions of the nodal coordinates $\mathbf{q}_{e}$ (see Equation (7.30)).

In general, this means that the elements in the mass matrix associated with the material coordinates $n_{i}$ and $m_{i}$ will retain their time dependency even if the material coordinates are kept fixed. In this way, the ALE-ANCF method differs from the conventional ANCF method in which the element mass matrix is constant for all element configurations. However, in some special cases, it is possible to modify the equations of motion such that the ALE-ANCF mass matrix remains constant. For instance, the mass matrix can be forced to remain constant in a case where all local material coordinates are intentionally kept fixed throughout the entire simulation. In these cases, it is possible to leave out the equations used to obtain solutions for the local material coordinates, and the time dependent terms of the mass matrix vanish. Then the system of equations reduces to a set that is consistent 
with the conventional ANCF method.

\subsection{Numerical example}

To demonstrate the ALE-ANCF method's ability to move a node progressively during a simulation, a simple model has been constructed. The model consists of four ALE-ANCF shell elements arranged in a $2 \times 2$ mesh to form a square plate with a side length of $1 \mathrm{~m}$ placed in the global $X Y$-plane. The plate is assumed to have a thickness of 0.01 $\mathrm{m}$. In the start configuration, the center node is shifted toward one of the plate corners as seen Fig. 6 .

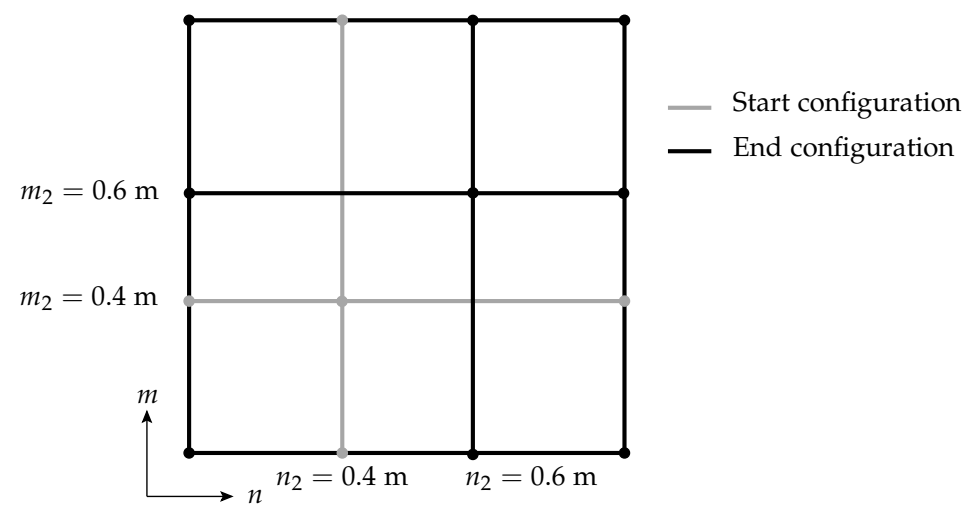

Fig. 6: ALE-ANCF model with moving node seen from the top.

A constant force acting in the negative $z$-direction acts on the center node, and the node moves diagonally at a constant velocity toward the opposite corner during simulation. The translational d.o.f. for the four corner nodes are fixed to ground and coincident nodes are connected using constraints that ensure both nodal connectivity and slope continuity between adjacent elements. It is important to note that this constraint based assembly technique is essential for making it possible to move the nodes in the mesh during a simulation. The center node is moved from $n, m=0.4 \mathrm{~m}$ to $0.6 \mathrm{~m}$ by imposing a displacement constraint on the $n$ and $m$ coordinates of the second row of nodes, that is $n_{2}$ and $m_{2}$. The constraint for the $n_{2}$-coordinate, $\Phi_{n}$, can be expressed as follows:

$$
\Phi_{n}=n_{2}-v \cdot t
$$


where $v$ is the velocity of the moving node. The constraint for the $m_{2}$-coordinate is expressed in a similar manner by replacing $n_{2}$ with $m_{2}$.

The ALE-ANCF model is validated by comparing its center node displacements with displacements obtained using corresponding thin ANCF shell elements with 36 d.o.f [19]. The displacements for comparison are obtained through static analyses where the center node is placed on the diagonal of the plate in a range from $n, m=0.4 \mathrm{~m}$ to 0.6 $\mathrm{m}$. Static solutions are obtained for five intermediate points. The plate is assumed to be made of linear elastic and isotropic material with a Young's modulus of $1 \times 10^{7} \mathrm{~Pa}$, a Poisson's ratio of 0.3 , and a density of $1000 \mathrm{~kg} / \mathrm{m}^{3}$. The applied force is $1 \times 10^{-3} \mathrm{~N}$. The simulation of the ALE-ANCF model begins from static equilibrium, and the node moves with a velocity of $0.01 \frac{\mathrm{m}}{\mathrm{s}}$. The low velocity is chosen to ensure near-static conditions in the ALE-ANCF model for valid comparison with the results from static ANCF solutions. Both sets of results are shown in Fig. 7. The plot shows that displacements obtained from the continuous simulation based on ALE-ANCF shell elements are comparable with the displacements from static solutions modeled with conventional ANCF elements.

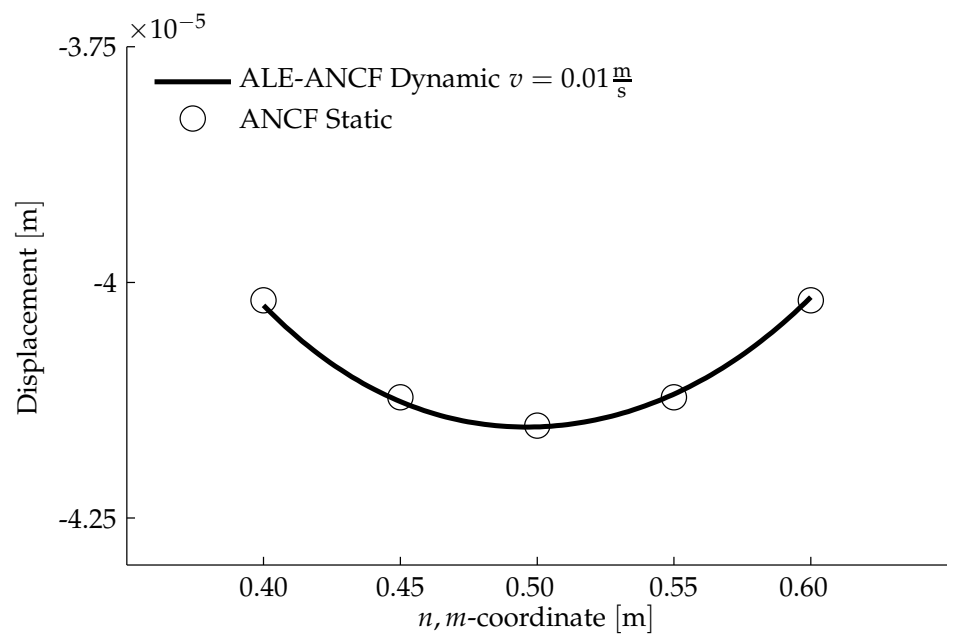

Fig. 7: Out of plane displacement of the moved node compared with static displacements. 
Another important physical quantity to validate is the strain level in the plate. To get an overall measure of the strain level in the model, the elastic energy from the dynamic simulation is compared to the elastic energy level resulting from the static ANCF analysis. These elastic energies are shown in Fig. 8 as a function of the position of the moving node. The elastic energy level from the model based on the ALE-ANCF method is consistent with the results obtained using the conventional ANCF method. This means that no additional strains are introduced by altering the node position in the mesh.

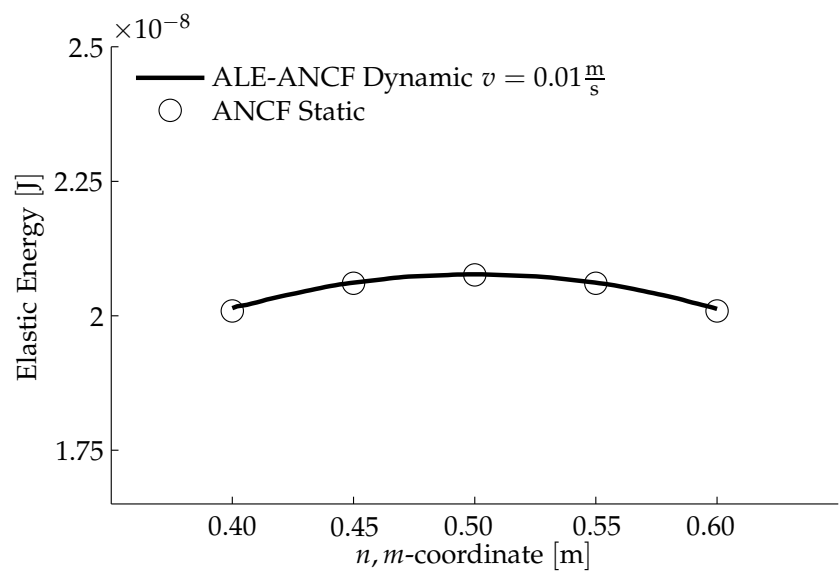

Fig. 8: The elastic energy in the model compared to energy levels obtained from static analysis.

\subsection{Discussion}

This study has shown that it is possible to formulate an ALE-ANCF shell element description that allows interior nodes to move progressively during simulation. The derivation of the ALE-ANCF shell description has revealed one major aspect where it differs from conventional ANCF element descriptions, namely the non-constant inertia description. Besides this difference, the derivation of elastic forces for the ALE-ANCF description follows the same procedure as in the conventional ANCF description.

The proposed ALE-ANCF method has been validated with two numerical studies where only one is presented here in this thesis. The first study, which is not shown here, demonstrates that the pro- 
posed ALE-ANCF method is consistent with the conventional ANCF method when ALE-ANCF elements are tied together using algebraic constraints, and relative node positions inside the specimen are kept fixed. This property is important if the method is to be applied in cases where movable nodes are only needed during some intervals of a simulation. For further explanation see the original research article [38] for the details regarding this first example. The second study presented here demonstrates the ability of the proposed ALE-ANCF method to move a node during simulation. In this simple study, a node was moved across the diagonal of a square plate with a constant velocity. The displacement in the direction normal to the plate and the elastic energy in the model were compared to results obtained using conventional ANCF elements with good agreement.

The scope of this study was to illustrate the method's ability to move a node progressively during transient simulation. This was accomplished using a simple academic example, but more advanced applications are easily imaginable. As mentioned in the introduction of the chapter, the method could be useful when modeling structures with moving boundary conditions such as traveling forces or sliding joints. Especially in the case of very thin structures with low bending stiffness, where a slope discontinuity will arise locally at the point where the force or sliding joint is acting. This discontinuity can easily be modeled using the constraint based assembly technique by omitting the constraint on the slope vectors.

It is also believed that the ALE-ANCF method could be used as extension to mesh refinement techniques. Using the ALE-ANCF, nodes could travel to areas where large deformation occurs to capture the deformation state more accurately. Such a technique could also be useful in contact problems. Here, the contact detection algorithm could trigger mechanisms that would force nodes to concentrate in the area where contact is expected.

Another application could be analysis of crack propagation in plate and shell structures. Taking, for instance, the model shown in Fig. 6, a crack could be initiated by omitting the node assembly constraint in one of the nodes on the boundary. This leaves an open gap between two of the elements with a node placed at the end, thus forming a crack. The node at the end of the crack could then be moved to resemble the crack propagating in the plate. 
A large number of enhancements is required before the ALE-ANCF method can be applied in any of these practical applications. In general, a sophisticated algorithm that makes is possible for a node to move all the way through the modeled specimen is needed. This algorithm must be formed in a way such that no numerical singularities are encountered when one node is approaching a neighboring node. In the numerical example shown here, the mesh was simple and consisted of only four elements. Further investigations must be made to clarify whether the ALE-ANCF method will work in a mesh with arbitrarily shaped elements. Specifically, it must be investigated whether the approach of only assigning each row and column of nodes a local material coordinate is sufficient. Alternatively, each node should be assigned its own local material coordinate, but this would increase the total number of d.o.f. drastically.

If the ALE-ANCF method is to be applied as a mesh refinement technique, some criteria are needed for determining when and which nodes should be moved and to which positions they should move. Furthermore, the criteria must be formulated in a way such that the solution is not compromised in other areas of the model. This could happen if, for instance, to many nodes travel to the area of interest, which would deteriorate the mesh quality in other areas.

The application of the ALE-ANCF in crack propagation analysis is maybe the most interesting. This would allow for simulating realistic component failure in the multibody dynamics framework. However, it would require a large research effort to derive and set up conditions on how and when the node at the crack tip should move, depending e.g. on the stress and strain state locally around this node or simply the forces acting at the node. Based on these state variables, the energy release rate can be calculated and used in evaluating a fracture criterion. Additionally, these criteria must be made in a way such that the crack does not close again.

Besides the needed enhancements tied to the ALE-ANCF method, some more basic problems were noticed. Numerical difficulties were observed when the aspect ratio of the ALE-ANCF elements varied significantly from 1 . These difficulties were noted at the beginning and the end of the simulation, where the numerical integrator used a smaller time step. Furthermore, it was problematic to find an appropriate model to use for validation of the ALE-ANCF method. Both re- 
sults from traditional commercial finite element codes and thin ANCF shell formulations were compared to the results from the dynamic simulation using the ALE-ANCF elements. The only successful validation was when the 36 d.o.f. element by [19] was used. This is remarkable since this element uses both the same kinematic and elastic description as the ALE-ANCF element presented here while the other elements differ in some way. This could indicate that a general difference can be expected when comparing results obtained using the different thin ANCF shell element formulations. This will be investigated further in the next chapter. 


\section{Part II}

\section{Behavior of thin ANCF shell elements in various mesh configurations}





\section{Behavior of thin ANCF shell elements in various mesh configurations}

The numerical validation of the ALE-ANCF shell element described previously in Section 7.4 shed light over some possible numerical problems which can be encountered when using thin ANCF shell elements. Here, it was noted how the numerical integrator was struggling when the elements had significant difference in size and non-optimal aspect ratios. Furthermore, it was difficult to compare results obtained using the ALE-ANCF shell element with other methods. The only successful validation was done by comparison with the 36 d.o.f. ANCF shell element by Dmitrochenko and Pogorelov [19] that uses the exact same kinematics and stiffness description. It is noticeable that significantly different results can be obtained when using different thin ANCF shell elements that use the same nodal d.o.f. and stiffness description but different interpolation shape functions. Such behavior clearly calls for further investigation.

Besides the observed behavior regarding numerical difficulties, other topics regarding the performance of thin ANCF shell elements need attention. So far, studies related to initially curved and distorted meshes of thin ANCF shell elements have been short of research focus. In general, most studies on ANCF shell elements have been based on purely academic models that, usually, are rectangular. One practical application of initially curved ANCF shell elements has been large deflection analysis of wind turbine blades $[7,56,8]$. The authors note some high frequency oscillations when the dynamic response from an ANCF model of a blade based on thin ANCF shell elements is com- 
pared to that obtained from a commercial finite element code. Especially in the case of a tapered blade profile, both high-frequency oscillations and a difference in the fundamental vibration frequency are noticed. The authors conclude that this behavior can be explained by the curved nature of the wind turbine blade and further investigation is needed to develop a good numerical model of a blade based on ANCF finite elements.

In this section, it is investigated how thin rectangular ANCF shell elements behave when used in different mesh configurations. Initially, a thorough comparison of the difference between available thin ANCF shell elements is carried out. Here, focus is on the difference in the used interpolation shape functions. Subsequently, a series of simple models is used to test the ability of current formulations to describe and analyze curved structures and how they perform when used in an irregular mesh. It is shown that current formulations are not capable of describing curved geometry without loss of inter-element connectivity at adjacent curved element edges. Numerical calculations also show that the accuracy of the results is reduced when a distorted finite element mesh is built using thin ANCF shell elements when compared to conventional finite element models. The findings in this part have been published in a journal paper [40] and a conference paper [39] authored by the author of this thesis and co-authors.

\section{Discussion on the kinematics of thin ANCF shell elements}

In this section, the kinematics of three thin ANCF shell elements is discussed. The discussion given here is more thorough than the one given previously in Section 5. This in-depth discussion is necessary since understanding of their individual characteristics is needed to interpret the differences in the results which will be presented later.

Traditional shell finite elements usually accommodate nodal displacements and two out-of-plane rotational d.o.f. [14]. For improved modeling of the element displacement field, additional d.o.f. based on a second order derivative can be added [101]. With this in mind, Dmitrochenko and Pogorelov formulated a 48 d.o.f. ANCF shell element [19]. This element uses the nodal positions, the two in-plane slope 
vectors, and a second order slope vector at each node as generalized coordinates as follows:

$$
\begin{aligned}
\mathbf{q}=\left[\mathbf{r}_{1}^{T}\left(\frac{\partial \mathbf{r}_{1}}{\partial x}\right)^{T}\left(\frac{\partial \mathbf{r}_{1}}{\partial y}\right)^{T}(\right. & \left(\frac{\partial^{2} \mathbf{r}_{1}}{\partial x \partial y}\right)^{T}, \\
& \left.\mathbf{r}_{4}^{T}\left(\frac{\partial \mathbf{r}_{4}}{\partial x}\right)^{T}\left(\frac{\partial \mathbf{r}_{4}}{\partial y}\right)^{T}\left(\frac{\partial^{2} \mathbf{r}_{4}}{\partial x \partial y}\right)^{T}\right]^{T}
\end{aligned}
$$

In their study, the interpolation shape functions are calculated using products of orthogonal Hermite beam shape functions [101]. The orthogonality of the shape functions implies that one set is a function of the local coordinate $x$ and the other set is a function of $y$. The four interpolation shape functions $\mathbf{N}$ associated with the nodal values at node $i$ are calculated as:

$$
\begin{aligned}
\mathbf{N}_{i}=\left[\begin{array}{lll}
H_{i}^{(0)}(x) \cdot H_{i}^{(0)}(y) & H_{i}^{(1)}(x) \cdot H_{i}^{(0)}(y) & \ldots \\
& H_{i}^{(0)}(x) \cdot H_{i}^{(1)}(y) & H_{i}^{(1)}(x) \cdot H_{i}^{(1)}(y)
\end{array}\right]
\end{aligned}
$$

where $H$ is the Hermite beam interpolation function. The superscript refers to the derivative order of the function and $x$ and $y$ are the local element coordinates. This approach is sometimes referred to as the crossed beam technique. An example of the practical calculation is given in [36].

As an alternative to the 48 d.o.f. element, Dmitrochenko and Pogorelov suggested that a reduced order element could be obtained by omitting the second order slope vectors from the generalized coordinates. Such an element was addressed by Dufva and Shabana [21]. By omitting the second order slope vector, the generalized coordinates reduce to:

$$
\mathbf{q}=\left[\mathbf{r}_{1}^{T}\left(\frac{\partial \mathbf{r}_{1}}{\partial x}\right)^{T}\left(\frac{\partial \mathbf{r}_{1}}{\partial y}\right)^{T} \ldots \mathbf{r}_{4}^{T}\left(\frac{\partial \mathbf{r}_{4}}{\partial x}\right)^{T}\left(\frac{\partial \mathbf{r}_{4}}{\partial y}\right)^{T}\right]^{T}
$$

The exclusion of the second order slope from the generalized coordinates has some advantages. First and foremost, the number of d.o.f. is reduced from 48 to 36 . Furthermore, the $\frac{\partial^{2} \mathbf{r}}{\partial x \partial y}$ slope vector has no clear geometrical meaning, which makes it difficult to interpret. 
The shape functions for the element presented by Dufva and Shabana are based on the following incomplete quartic polynomial:

$$
\begin{aligned}
\mathbf{r}=\mathbf{a}_{1}+\mathbf{a}_{2} x+\mathbf{a}_{3} y+\mathbf{a}_{4} x^{2}+\mathbf{a}_{5} x y+\mathbf{a}_{6} y^{2}+\ldots \\
\mathbf{a}_{7} x^{3}+\mathbf{a}_{8} x^{2} y+\mathbf{a}_{9} x y^{2}+\mathbf{a}_{10} y^{3}+\mathbf{a}_{11} x^{3} y+\mathbf{a}_{12} x y^{3}
\end{aligned}
$$

where $\mathbf{a}_{i}$ are vectors of polynomial constants which will vary in time in the case of transient simulations. A set of interpolation shape functions, $\mathbf{N}$, that depends on the local element coordinates $x$ and $y$ and the element length $L$ and width $W$ can be obtained using matrix operations. An example of this can be found in [36].

In addition to the two elements mentioned above, an additional 36 d.o.f. element is considered in this study. It uses the nodal d.o.f. as stated in Equation (8.40) and shape functions based on the crossed beam technique. However, to match the fewer number of d.o.f., the last term in Equation (8.39) is omitted from the shape function matrix. This leads to a slightly different 36 d.o.f. element than the one treated by Dufva and Shabana due to the different approach for deriving the shape functions.

The three elements described above fall into two categories. The first category contains the elements described in the work of Dmitrochenko and Pogorelov [19] that use the crossed beam technique for deriving the element shape functions. The last is the element by Dufva and Shabana [21] that bases the element kinematics on a polynomial expansion. These two methods for deriving the element shape functions are fundamentally different and this affects the resulting kinematic description.

As mentioned, Dmitrochenko and Pogorelov based their element kinematics on the crossed beam technique and Hermite beam shape functions which have a certain characteristic. Using a Hermite polynomial of order $2 n+1$ leads to $C^{n}$ continuity between adjacent elements. Based on the chosen generalized coordinates, a third order Hermitian interpolation is applied. This leads to shape functions that will ensure a $C^{1}$ continuous displacement field. Since these $C^{1}$ continuous beam shape functions form the basis of the ANCF shell element, the resulting shell shape functions will also be $C^{1}$ continuous [101].

The shape functions used in the 36 d.o.f. element by Dufva and Shabana are based on an incomplete quartic polynomial (see Equa- 
tion (8.41)). This polynomial is incomplete since the terms $x^{4}, y^{4}$, and $x^{2} y^{2}$ are missing. As a result, the displacement along any edge of the element will vary as a cubic polynomial which can be uniquely defined when using both the position and slope vectors as nodal d.o.f. The slopes at any element edge will vary as a second order polynomial, but since only two slope vectors normal to any element edge are utilized, one at each node, this polynomial is not unique. These two characteristics mean that inter-element displacement continuity is ensured, but inter-element slope continuity, in general, is not [101]. This means that the displacement field is $C^{0}$ continuous.

This difference in the order of ensured displacement continuity is important for the behavior of the thin ANCF shell elements. In the literature, $C^{1}$ continuity is mentioned as a theoretically indispensable requirement when the element stiffness properties are calculated based on element curvature [101,29]. This is due to the fact that the curvature of the overall displacement field will be infinite at the boundary between two adjacent elements if there is a discontinuity in the slope. This can be an issue for the thin ANCF shell elements where the Kirchhoff theory is utilized, which requires calculation of the curvature of the displacement field (See Equation (6.21)). This could lead to differences when comparing the $C^{1}$ continuous element with the $C^{0}$ continuous element. Whether such a difference is present will be investigated further in the next sections.

\section{Convergence study on model with optimal mesh}

To initiate the investigation on the behavior of different thin rectangular ANCF shell elements, a convergence study is performed on a simple rectangular plate modeled by a uniform mesh. This study will both highlight any differences between the compared elements and act as a benchmark for the following studies. The different element formulations that will be treated throughout this and the subsequent investigations are the 48 d.o.f. $C^{1}$ continuous element, the 36 d.o.f. $C^{1}$ continuous element, and the 36 d.o.f. $C^{0}$ continuous element. The formulations are implemented directly as described in Section 6 without taking into account known defects such as membrane locking (see [66]). This is done intentionally for the sake of simplicity. 
Behavior of thin ANCF shell elements in various mesh configurations

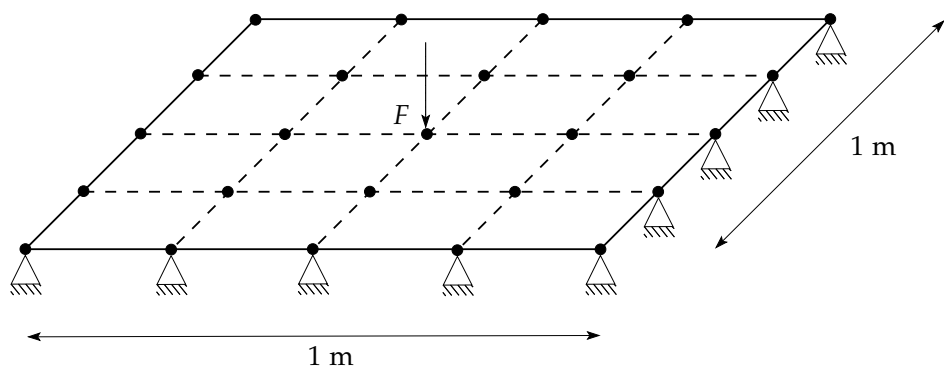

Fig. 9: Simply supported square plate divided into a uniform mesh with a point force acting at the center of the plate.

For the convergence study, the deflection of a plate subjected to a transverse force acting in the center of the plate is investigated. This is shown in Fig. 9. A simply supported boundary condition is applied by fixing all displacements and slope vectors parallel to the plate edges at the boundary nodes while the slope vectors perpendicular to the free edges are kept free allowing the edges to rotate. The structural properties of the plate are given in Table 1 The center point deflection is calculated for several mesh sizes using all three formulations for three load cases: $F=0.1 \mathrm{~N}, 1.0 \mathrm{~N}$, and $10 \mathrm{~N}$.

Before discussing the convergence study, the difference in continuity order ensured by the applied interpolation, $C^{0}$ versus $C^{1}$, is demonstrated. This is done by comparing the calculated deflections. The deformed square plate is shown in Fig. 10 for the two models analyzed with 36 d.o.f. elements. The deformed mesh for the 48 d.o.f. $C^{1}$ continuous element is not shown here because it is identical to the one obtained using the 36 d.o.f. $C^{1}$ continuous element. The models shown have a mesh size of $2 \times 2$ elements. By inspecting Fig. 10a, it is

\begin{tabular}{lr}
\hline Property & Value \\
\hline Side lengths & $1 \mathrm{~m}$ \\
Thickness & $0.01 \mathrm{~m}$ \\
Young's Modulus & $1 \times 10^{7} \mathrm{~Pa}$ \\
Poisson's ratio & 0.3 \\
\hline
\end{tabular}

Table 1: Parameters for the simply supported square plate to convergence study. 
9. Convergence study on model with optimal mesh

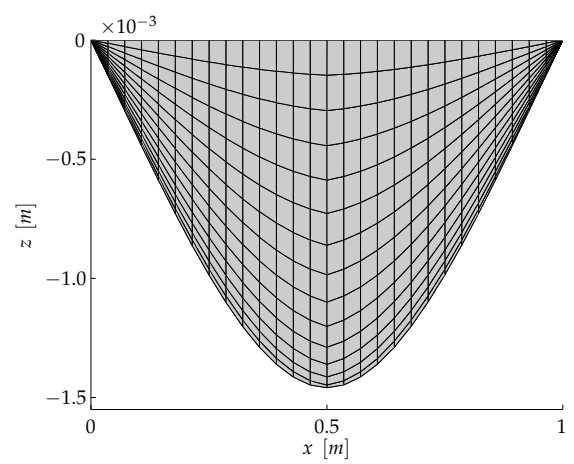

(a) 36 d.o.f. $C^{0}$ continuous elements.

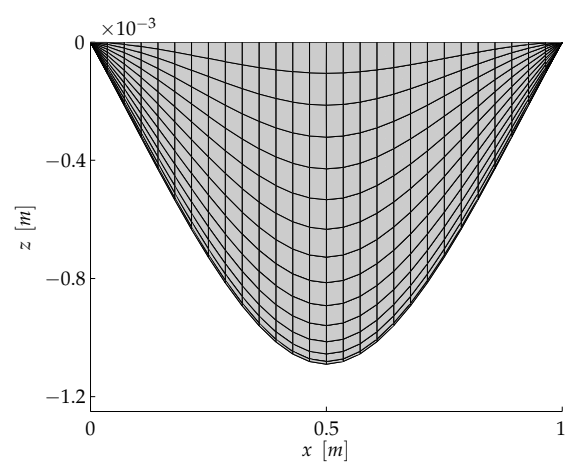

(b) 36 d.o.f. $C^{1}$ continuous elements.

Fig. 10: Sideviews of the deformed meshes for the square model with $2 \times 2$ elements subjected to a center load with $F=0.1 \mathrm{~N}$. Note the discontinuity of the trend lines at $x=0.5 \mathrm{~m}$ for the model analyzed with the 36 d.o.f. $C^{0}$ continuous elements.

seen how the trend lines of the model using $C^{0}$ continuous elements are discontinuous at $x=0.5 \mathrm{~m}$, which is at the interface of the two visible elements. When studying the figure with the $C^{1}$ continuous elements (Fig. 10b), the observation is different. Here, the transition from one element to the other is nice and smooth. Furthermore, just by qualitative comparison of the maximum deflection, it is seen that there is a large difference in the predicted displacement. This is remarkable since the models use the same material parameters, boundary conditions, generalized nodal coordinates, and stiffness description. This clearly demonstrates the difference between using $C^{1}$ versus $C^{0}$ continuous interpolation shape functions.

For the convergence study, results from the ANCF models are compared to corresponding results obtained using the commercial finite element solver MSC Nastran. Using MSC Nastran, a similar model is analyzed using their eight noded CQUAD8 elements and solved using the implicit nonlinear SOL400 solution sequence [58]. The results from all three ANCF models are shown in Fig. 11 together with the results from MSC Nastran. The results clearly show that there is a general difference between the formulations since both of the $C^{1}$ continuous elements converge to a lower deflection than the $C^{0}$ continuous element. Furthermore, in all three load cases, the $C^{0}$ continuous element converges to a result comparable to that obtained by the conventional 
Behavior of thin ANCF shell elements in various mesh configurations

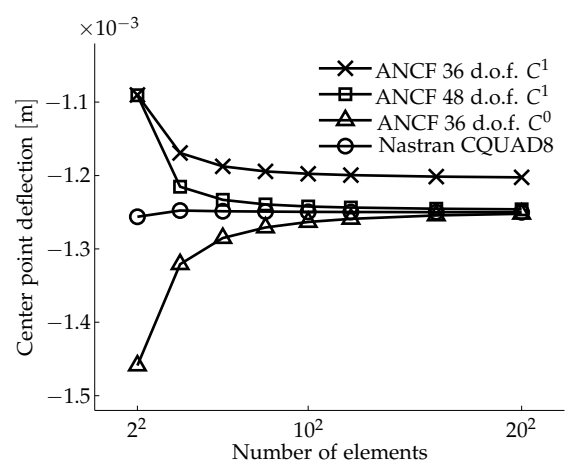

(a) Center point deflection, $\mathrm{F}=0.1 \mathrm{~N}$.

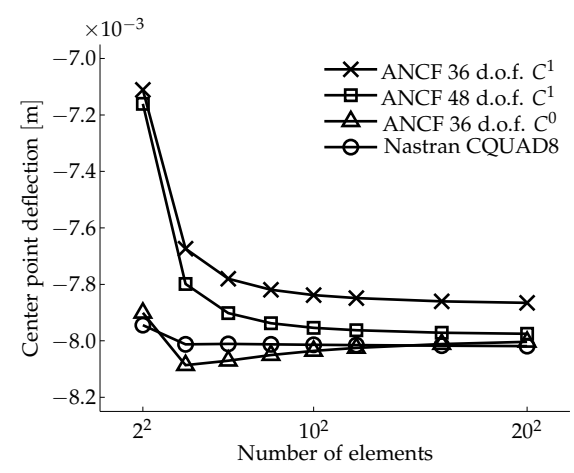

(b) Center point deflection, $\mathrm{F}=1.0 \mathrm{~N}$.

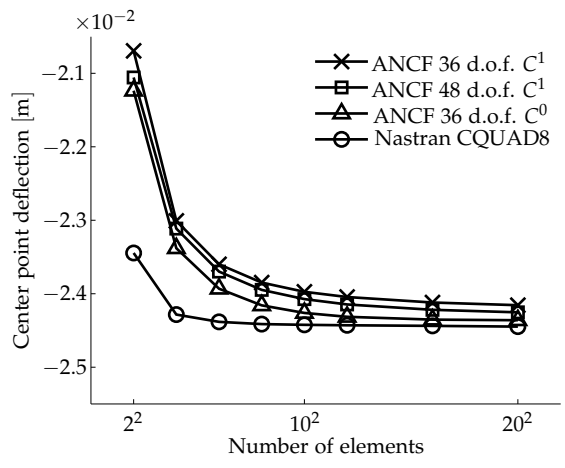

(c) Center point deflection, $\mathrm{F}=10 \mathrm{~N}$.

Fig. 11: Deflections measured at the center point of the simply supported square plate for varying loads.

CQUAD8 elements. It is also noticeable that in the load case where $F=0.1 \mathrm{~N}$, the $C^{0}$ continuous element heavily over-predicts the deflection for a coarse mesh (see Fig. 11a). This trend seems to wane as the load increases, finally converging in the same pattern as the $C^{1}$ continuous elements at $F=10 \mathrm{~N}$ (see Fig. 11c). Another interesting tendency is that the 48 d.o.f. $C^{1}$ continuous element performs significantly better than the corresponding 36 d.o.f. element, especially in the two load cases with $F=0.1 \mathrm{~N}$ and $F=1.0 \mathrm{~N}$. Here, the 48 d.o.f. element converges to a result closer to the results from both MSC Nastran and the 36 d.o.f. $C^{0}$ continuous element. This better performance over the 36 d.o.f. $C^{1}$ continuous element suggests that important polynomial terms are excluded from the shape functions when omitting the sec- 
10. Influence of non-uniform element sizes on model stiffness

ond order slope vectors from the generalized coordinates. In general, the ANCF models converge much more slowly than the conventional CQUAD8 element models, meaning that a significantly higher number of elements are needed to obtain a converged solution. This can be explained by the fact that membrane locking has not been alleviated in the analyzed models, but no studies to support this explanation have been made.

\section{Influence of non-uniform element sizes on model stiffness}

In the discussion about the ALE-ANCF shell element (see Section 7.4), it was mentioned that the numerical integrator was struggling when the element aspect ratios, that is $L_{\max } / L_{\min }$, in the model were different from one. This behavior indicates that the analyzed ANCF shell elements perform bad when they have large aspect ratio. This can cause problems if the thin ANCF shell elements are to be applied in the design of real life structures. Here, it is inevitable to create models made of only optimal elements with aspect ratios close to one. An example of this could be models where a fine mesh is created in areas of high interest and a coarser mesh in other regions of low interest. This will lead to elements with high aspect ratios in the transition zone from the fine to the coarse mesh. It is widely known that finite element models containing elements with large aspect ratios will affect the simulation result. Furthermore, abrupt changes in element size will lead to disturbances in the position gradient field [14]. In this section, it will be studied how the thin ANCF shell elements behave when applied in models with non-optimal mesh configurations, and how they perform compared to conventional shell finite elements.

The sensitivity of the ANCF shell elements to varying aspect ratios and abruptly changing element sizes is investigated by studying the deflection of a simple structural model subjected to static loading. The model used in this study is a simple cantilever rectangular plate (see Fig. 12). The plate is subdivided into four areas, and the shape of the areas is altered by shifting the common point towards point $a$ along the plate diagonal. This changes the aspect ratio of each area (see Fig. $12 b)$. When each of the areas are discretized with finite elements, the 
Behavior of thin ANCF shell elements in various mesh configurations

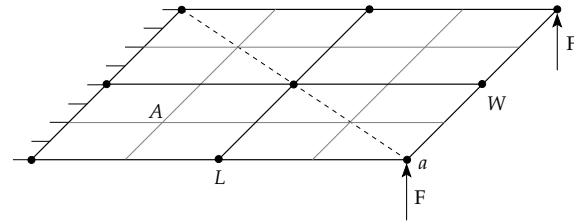

(a) Uniform element aspect ratio.

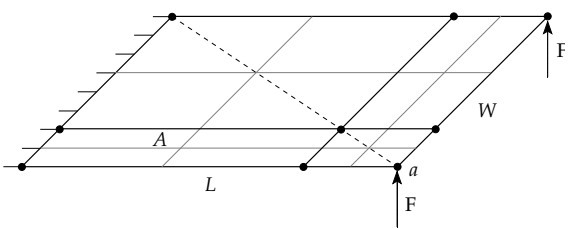

(b) Non-unifrom element aspect ratio.

Fig. 12: Cantilever plate with transverse loading at corner points. The transverse deflection of point $a$ is monitored as the aspect ratio of the elements in area A varies. The dashed line indicates the diagonal of the plate and the grey lines indicate the finite element discretization. The plate is shown with a $4 \times 4$ element discretization.

finite elements will have the same aspect ratio as the area in which they are located, respectively.

The cantilever plate is assumed to be $L \times W \times h=6 \mathrm{~m} \times 2 \mathrm{~m} \times$ $0.01 \mathrm{~m}$ and made of a linear elastic isotropic material with a Young's modulus of $1 \times 10^{7} \mathrm{~Pa}$ and a Poisson's ratio of 0.3 . This means that when each of the areas are of equal size, their aspect ratios will be $\mathrm{L}_{\max } / \mathrm{L}_{\min }=3$. A fixed boundary condition is applied by constraining the positions and slope vectors of the nodes at the leftmost edge of the plate, and a transverse load, F, is applied at each of the two free corners (see Fig. 12). The transverse deflection is monitored at point $a$ for each mesh configuration. Furthermore, deflections for the cantilever model are calculated with the commercial finite element solver MSC.Nastran using both CQUAD4 and CQUAD8 elements [58].

As the purpose of this investigation is to illustrate how the mesh configuration affects the simulation results and not to evaluate the numerical precision of each formulation, the results have been normalized with respect to the deflection calculated with the uniform mesh. This means that the plot will show how rapidly the results will degrade as a function of the element aspect ratio. The sensitiveness is studied for models consisting of $2 \times 2,4 \times 4$, and $6 \times 6$ elements distributed equally in the four areas. A mesh composed of $4 \times 4$ elements is shown in Fig. 12. The normalized deflections are shown in Fig. 13 as a function of the aspect ratio of elements in area $A$ (see Fig. 12).

In the first case where a mesh consisting of $2 \times 2$ elements has been used, the simple four-noded element CQUAD4 seems unaffected and the deflections calculated using the CQUAD8 elements decrease only 
10. Influence of non-uniform element sizes on model stiffness

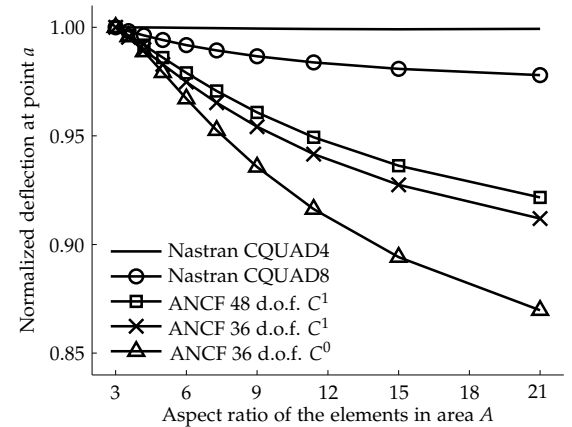

(a) Deflections for the model using $2 \times 2$ shell elements.

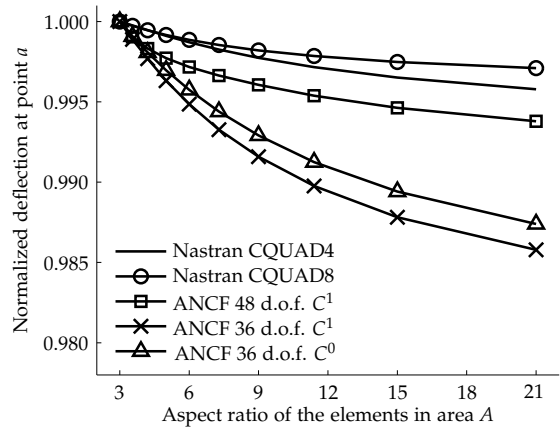

(b) Deflections for the model using $4 \times 4$ shell elements.

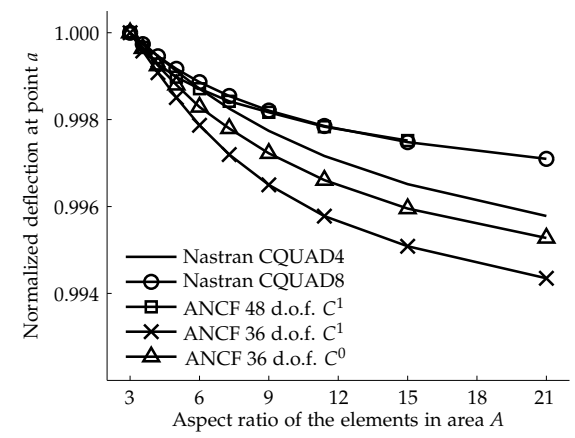

(c) Deflections for the model using $6 \times 6$ shell elements.

Fig. 13: Normalized deflection of the corner of a cantilever plate as a function of the element aspect ratio.

slightly when compared to the reference based on the uniform mesh configuration. The deflections of the ANCF based models, on the other hand, diverge rapidly from their calculated reference deflection when the mesh turns irregular. This indicates that the overall stiffness of the ANCF models increases rapidly for an increasingly irregular mesh configuration, leading to an under-prediction of the actual deflection. It is also noticeable that the $C^{0}$ continuous element seems more sensitive to the change in aspect ratio than the two $C^{1}$ continuous elements. In addition to the increased stiffness, increased computational time is noticed for all of the ANCF formulations with an increasing irregular mesh. Specifically, an increased number of iterations was needed in 
the solution process to acquire a converged solution.

When additional elements are introduced in the finite element discretization, the results improve significantly. In the case of a mesh consisting of $4 \times 4$ elements, the results from the two 36 d.o.f. elements are only degraded less than $1.5 \%$ in the worst case and approximately $0.5 \%$ in the case where $6 \times 6$ elements have been used. A more interesting tendency is that the 48 d.o.f. ANCF element is improving relative to the conventional finite elements, and in the case with the $6 \times 6$ elements, it is performing as good as the CQUAD8 element in Nastran. It should be noticed, though, that it was not possible to obtain a converged solution for an aspect ratio of 21 with the 48 d.o.f. $C^{1}$ continuous element. Furthermore, it took several tries to select a proper perturbation parameter for calculation of the finite differences used to derive the tangent stiffness matrix (see Equation (6.22)) before it was possible to obtain the results successfully.

Overall, this simple study indicates that ANCF-based models appear more sensitive to irregular mesh configurations than conventional finite elements when an insufficient number of elements is used. This tendency seems to decrease, though, when more elements are used. In this simple example, all formulations converged rapidly which may explain why simulation results are satisfactory after only one mesh refinement. In other more complex models, the tendency may not decrease a fast as in this simple case. Therefore, it is suggested that ANCF-based meshes containing irregular elements and/or elements with a large aspect ratio should be used with caution. Thorough convergence studies must be performed in order to obtain a numerical model of good quality.

\section{Representation of initially curved structures}

Two different approaches have been used to create a set of interpolation shape functions for thin ANCF shell elements. Both of these methods and the choice of base functions are consistent with those used to develop Kirchhoff shell elements. The kinematics of this element is thoroughly discussed in the literature (see for instance [48]). In the discussion, several problems are mentioned regarding the kinematics of this element type. Shape functions based on the crossed beam tech- 
nique lack the ability to describe constant twist. Furthermore, the element shape functions derived from the incomplete quartic polynomial are known to have compatibility problems. A more concerning fact is that this type of element cannot be used to describe non-rectangular shapes [14]. This is a problem since engineering problems, in general, cannot be discretized entirely into rectangular shapes.

The lack of ability to describe non-rectangular shapes can be illustrated by discretizing a plane hollow disc by thin ANCF shell elements. This is depicted in Fig. 14. The elements applied for the discretization shown in Fig. 14 are 36 d.o.f. $C^{1}$ continuous elements. However, similar figures can be obtained by using the 48 d.o.f. $C^{1}$ continuous element or the 36 d.o.f. $C^{0}$ continuous element which will lead to the same observations and conclusions drawn in this section.

It is clear that the elements do not cover the entire physical disc as there are gaps between adjacent curved element sides. This leads to a non-continuous description of the displacement field. It is noticeable that it is only between curved faces that inter-element connectivity is lost while the connectivity between straight sides is maintained. For qualitative comparison, a discretization of the disc obtained using conventional eight noded shell elements (Q8 elements) [14] is shown in Fig 15. Here, it is seen that a much more accurate representation of the actual geometry is obtained. This is remarkable since the shape functions used by the Q8 elements are based on the same incomplete quartic polynomial as used to derive the shape functions for the $C^{0}$ continuous element (see Equation (8.41)). The difference between the two discretizations lies in that the ANCF elements rely on the slopes at the nodes to describe the circle segment lying between the nodes whereas the Q8 elements have nodes on the middle of the sides for better description of curved structures.

Further conclusions can be drawn by closer inspection of the gap between elements 3 and 4 in the ANCF discretization. Comparing the element edges between nodes 5 and 8 to the segment of the circle they are meant to describe, it is seen that the edges lie on either side of the circular segment (see Fig. 16a). Furthermore, by comparing the distances measured perpendicular from the true circle to the element edges (see Fig. 16b), it is seen that the gap is the largest at the middle of the element span and is nearly the same for the two elements but with an opposite sign. The negative distance means that the edge lies 


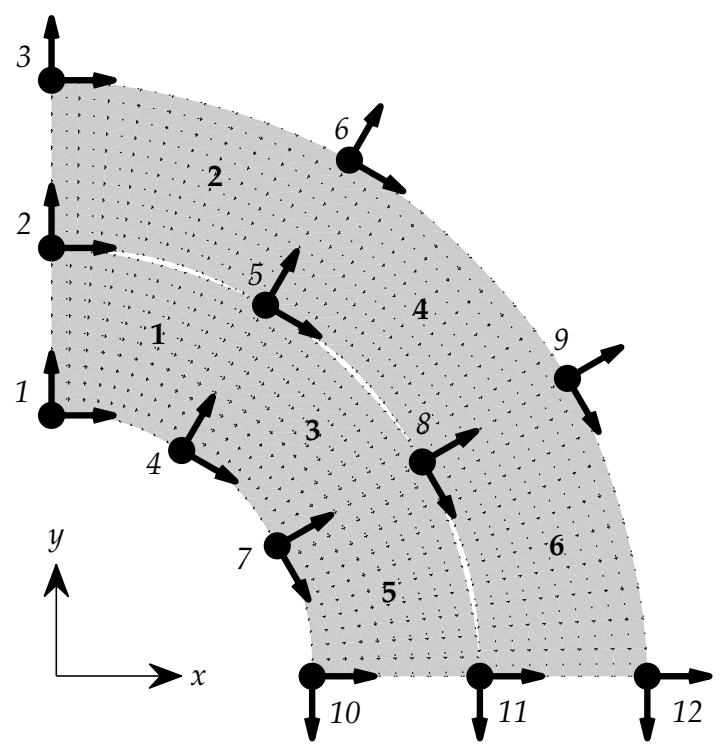

Fig. 14: A hollow disc with an inner radius of $3.5 \mathrm{~m}$ and an outer radius of $8 \mathrm{~m}$ discretized by six thin rectangular ANCF shell elements. The nodes are shown with their corresponding slope vectors, where the tangent vectors of the circle are the $\frac{\partial \mathbf{r}}{\partial x}$ and the radial vectors represent the $\frac{\partial \mathbf{r}}{\partial y}$. Node numbers are given in italic and element numbers are given in bold. Note the gap between curved element sides.

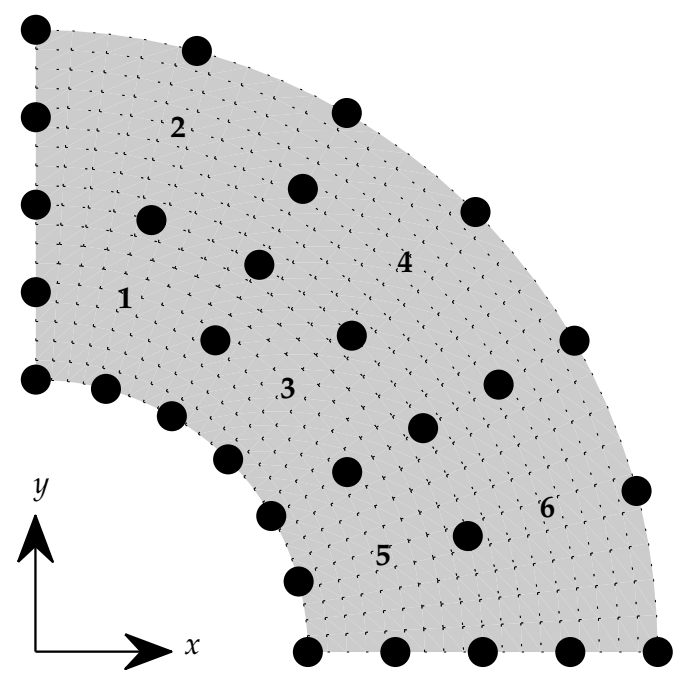

Fig. 15: A hollow disc with an inner radius of $3.5 \mathrm{~m}$ and an outer radius of $8 \mathrm{~m}$ discretized by six conventional eight noded shell elements (Q8 elements). The element numbers are given in bold while the node numbers are omitted for brevity. 
on the inside of the true circle. The trend with the symmetric gap may not be the same in the case of arbitrarily curved geometries.

The Q8 element discretization has been subjected to the same inspection and the results are shown in Fig. 17. Here, it is noted that the two element edges are coincident along the entire element span, meaning that there is no gap between the elements, and the displacement field is continuous. Furthermore, by comparing Fig $16 \mathrm{~b}$ and Fig. $17 \mathrm{~b}$, it is noted that the maximum distance from the elements to the true circle is roughly $8 \times 10^{-4} \mathrm{~m}$ in the model using Q8 elements while it is $4 \times 10^{-2} \mathrm{~m}$ when the ANCF elements are used.

Another interesting tendency can be identified by closer inspection of the evaluation points used to plot the element edges in the ANCF discretization (see Fig. 16a). Here, the ticks on the curves, representing the edges of the elements, mark the position of the evenly distributed evaluation points obtained by interpolation of the nodal coordinates. The thin lines perpendicular to the true circle mark equally spaced evaluation points on the circle. It is seen that the positions of the evaluation points on the edges of the elements differ from the equally spaced points on the true circle. Furthermore, the spacing between the evaluation points on the elements is non-uniform. This stands in contrast to the distribution of the evaluation points obtained using the Q8 elements (see Fig. 17a) where the evaluation points on both edges coincide with the equally spaced lines on the true circle.

This characteristic regarding non-uniform spacing of interpolated evaluation points in the thin ANCF shell elements has been noted previously [66]. Here, this phenomenon was labeled curve induced distortion (CID) and it was concluded that it causes membrane locking in thin ANCF shell elements. CID occurs in curved elements because the use of polynomial-based shape functions leads to a uneven distribution of interpolated points. As a consequence, slopes of a non-straight position field are not unit vectors and the norms of the slope vectors vary even though the slopes defined at the nodes are unit vectors. A mathematical discussion of the topic is given in [23, 24].

For the particular model used in this study, the $l^{2}$-norms of the slope vectors evaluated at the edges of element 3 and 4 between nodes 5 and 8 are shown in Fig. 18. The norm of the slopes are related to the strains in the element as seen in Equation (6.20). According to the equation, a slope norm larger than 1 corresponds to a state of tension 


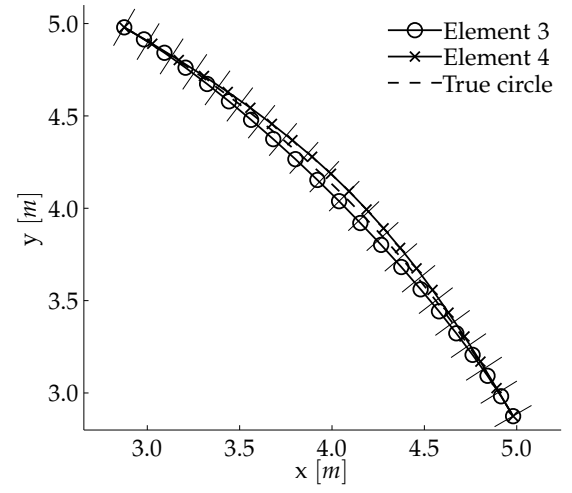

(a) Adjacent element edges for element 3 and 4 plotted together with the geometrically true circle they are trying to describe.

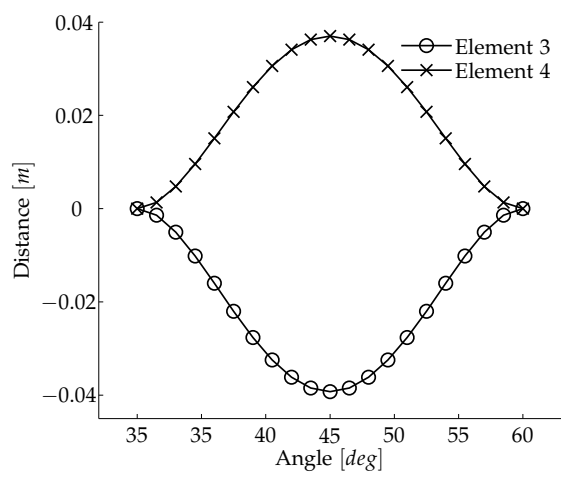

(b) Distance from curved element edge measured in the direction perpendicular to the true circle shown as a function of the span angle. The angle is measured counter clockwise starting from the $x$-axis.

Fig. 16: Analysis of gap between elements 3 and 4 in the ANCF discretization.
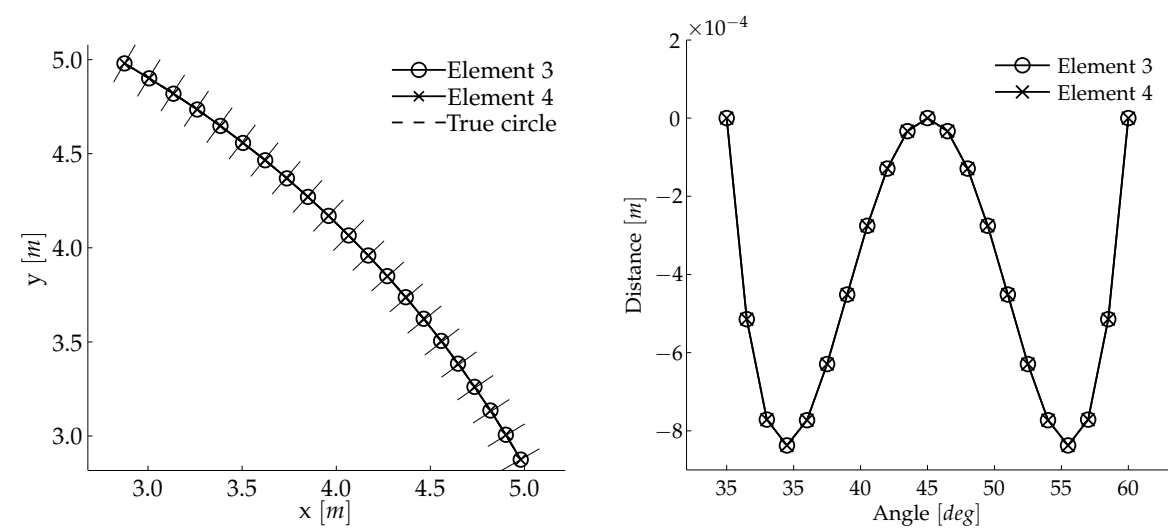

(a) Adjacent element edges for element 3 (b) Distance from curved element edge and 4 plotted together with the geometrically true circle they are trying to describe. measured in the direction perpendicular to the true circle shown as a function of the span angle.

Fig. 17: Analysis of gap between elements 3 and 4 in the Q8 discretization. 


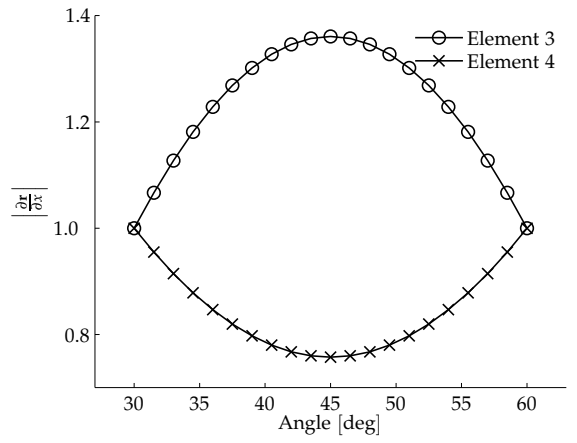

(a) $l^{2}$-norm of the slope vector $\frac{\partial \mathbf{r}}{\partial x}$.

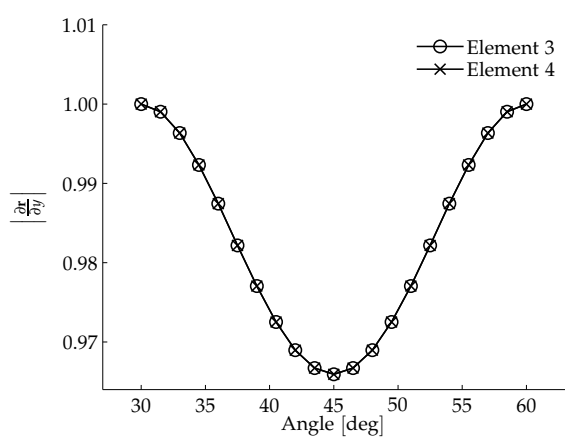

(b) $l^{2}$-norm of the slope vector $\frac{\partial \mathrm{r}}{\partial y}$.

Fig. 18: Norms of slope vectors at the element edges on the interface between elements 3 and 4 for the ANCF discretization. $\frac{\partial \mathbf{r}}{\partial x}$ is the vector in tangential direction and $\frac{\partial \mathbf{r}}{\partial y}$ is the radial vector as shown in Fig. 14

and a norm less than 1 corresponds to compression. Hence, at the interface between elements 3 and 4, element 3 is stretched and element 4 is compressed (see Fig. 18a). Furthermore, by inspection of the norm of the slopes perpendicular to the element interface $\left|\frac{\partial \mathbf{r}}{\partial y}\right|$, it is seen that both elements are contracted to the same level and the contraction is largest at the middle of the element edges. This corresponds well to the elements depicted in Fig. 14 where the elements are bent into curved shapes and contracting in the radial direction, thus creating a gap between the elements. This bending behavior is partly due to the fact that the shape functions of the elements are derived for a shell in a rectangular configuration. In this case, the shell element is assumed to have a constant length and width at all evaluation points. When the element is used in a non-straight initial configuration, the elements will have non-constant lengths and widths in their undeformed states. It can be shown that if the correct element lengths and widths corresponding to the actual geometry are used in the evaluation of the element shape functions, the element will resemble a curved geometry without any gaps between adjacent elements, and the slopes of the position field are constant unit vectors. This will be demonstrated in Section 13. 
Behavior of thin ANCF shell elements in various mesh configurations

\section{Convergence study on initially in-plane curved structures}

The reasons for the ANCF shell element's lacking ability to describe a curved geometry were discussed in the previous section. The complications of the discontinuous displacement field are the focus of this section. An inherently derived flaw from the discontinuous kinematic description of an initially curved geometry is poor modeling of the mass and inertia properties of such curved structures. This is due to the fact that the material left in the gaps will not contribute to the mass and inertia of the discretized model. It can be shown, however, that the error in the inertia modeling will diminish when the mesh is refined sufficiently because the gaps will become smaller as the size of the elements is reduced.

A second and more important concern is that the loss of interelement displacement continuity may affect the stiffness description of the discretized structure. The problem regarding gaps and displacement discontinuities between finite elements is not new and elements that show this characteristic are known as non-conforming or incompatible elements [14]. In many cases, this characteristic is unproblematic as the gaps between elements reduce as the mesh is refined. In matter of fact, in some particular cases, a non-conforming element can have significantly better performance than a similar conforming one [101]. Furthermore, incompatible modes can be added to an element displacement field to remedy locking [14]. However, incompatibility can also result in convergence problems, lacking ability to pass the patch test, and shape sensitive performance $[48,101]$.

To test whether the gaps have any influence on solutions obtained using the three thin ANCF shell elements, a convergence study is performed on the hollow disc discussed in the previous section. A sketch of the model is shown in Fig. 19. Due to symmetry with respect to both the $X Z$ and $Y Z$ planes, the model is reduced to only one quarter of the full disc. A simply supported boundary condition is applied at the outer edge by fixing the translational d.o.f. of the nodes. At the symmetry planes, the $\mathrm{z}$-components of the $\frac{\partial \mathrm{r}}{\partial x}$ and the translational 
12. Convergence study on initially in-plane curved structures

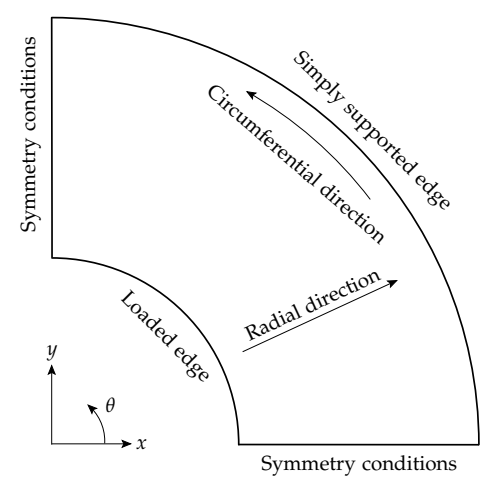

Fig. 19: Schematics of the quarter hollow disc model.

d.o.f. perpendicular to the symmetry plane ${ }^{2}$ are fixed to enforce the symmetry conditions to the model. An evenly distributed load acting in the $\mathrm{z}$-direction is applied to the inner edge. The model parameters are shown in Table 2.

The convergence study is performed for four load cases with an increasing magnitude. For all load cases, the transverse deflection of the loaded edge is monitored and compared to solutions obtained by the implicit non-linear SOL400 in MSC Nastran using CQUAD8 elements. In all cases, the computational mesh is made such that there are twice as many elements in the circumferential direction as in the radial direction. The results from the convergence study are shown in Fig. 20.

\begin{tabular}{lr}
\hline Property & Value \\
\hline Outer radius & $8 \mathrm{~m}$ \\
Inner radius & $3.5 \mathrm{~m}$ \\
Thickness & $0.025 \mathrm{~m}$ \\
Young's Modulus & $1 \times 10^{9} \mathrm{~Pa}$ \\
Poisson's ratio & 0.3 \\
\hline
\end{tabular}

Table 2: Parameters for the circular model subjected to convergence study.

${ }^{2}$ Displacements in the $y$-direction are restrained for the nodes along the edge parallel to the $\mathrm{x}$-axis, and displacements in the $\mathrm{x}$-direction are restrained for the nodes along the edge parallel to the $y$-axis 
Behavior of thin ANCF shell elements in various mesh configurations
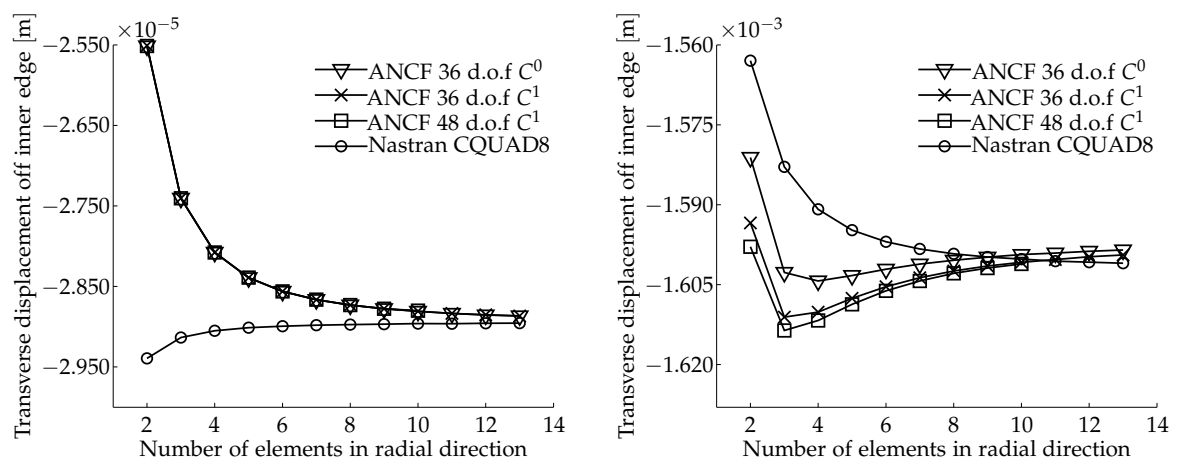

(a) Convergence plot for a total load of $-1 \times 10^{-5} \mathrm{~N}$.

(b) Convergence plot for a total load of $-1 \times 10^{-3} \mathrm{~N}$.
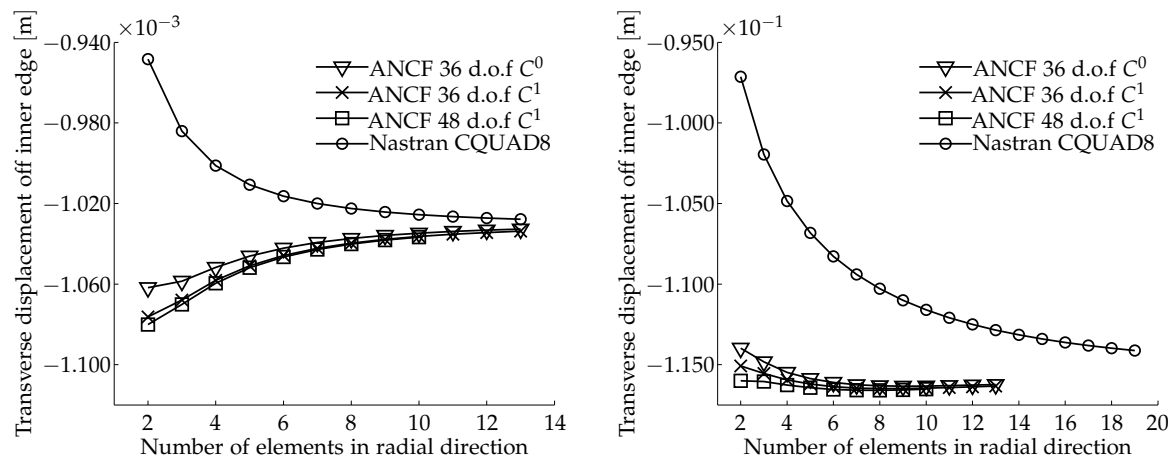

(c) Convergence plot for a total load of $-1 \times 10^{-1} \mathrm{~N}$.

(d) Convergence plot for a total load of $-1 \times 10^{2} \mathrm{~N}$.

Fig. 20: Convergence study on a simply supported hollow disc for four different load cases.

By inspection of the convergence plots, some interesting phenomena can be observed. For the first load case, a linear analytical solution for an axisymmetrical circular plate in pure bending [61] gives an exact result of $-2.896 \times 10^{-5} \mathrm{~m}$. The ANCF models predict displacements that are slightly lower than the analytical solution, and the results from MSC Nastran converge to the exact result. As the load on the circular disc model is increased, the ANCF appears to converge to an increasingly larger deflection than the corresponding results from the conventional finite element models. This stands in contrast to the previous observations. In the convergence study on the rectangular model 
conducted in Section 9, the ANCF models continued to under-predict the magnitude of the deflections when the loads were increased (see Fig. 11). This tendency is unexpected as the thin ANCF shell elements are known to exhibit membrane locking [66] which should lead to an underprediction of the deflection as membrane deformations are increased. In general, the convergence characteristics of the model based on the initially curved ANCF shell elements are different from those seen in the case of a purely rectangular and uniform mesh. This indicates that the convergence of models containing thin ANCF shell elements depends on the initial shape of the elements.

During the preparation of the results for the convergence analysis on the hollow disc model, some numerical problems were noted. As explained previously, the static solutions are obtained using an iterative solution technique as described in Equation (6.22), where the tangent stiffness matrix is calculated numerically using finite differences. When switching from the model containing only rectangular elements to the model with initially curved elements, it was considerably more difficult to choose a perturbation parameter that would give stable and converged solutions. Especially for the models subjected to larger loads, it was necessary to choose the perturbation parameter individually for each mesh configuration. This further supports the previous findings in Section 10 where the same tendency regarding difficulties in selecting proper solution parameters for the static equilibrium solution algorithm was noted.

Additionally, it was observed that the models with the larger applied loads produced uneven deformations at the loaded edge. Clearly, these results were not in agreement with the applied uniform load. It was found that by increasing the number of sampling points used in the Gaussian quadrature to obtain the vector of elastic forces from $3 \times 3$ to $5 \times 5$, the deflection of the loaded edge became more even. The increase in sampling points had another beneficial effect since the analysis converged at a significantly lower number of iterations. This suggests that the ANCF shell elements are sensitive to the order of the quadrature rule.

Based on the results shown in Fig. 20, it is concluded that ANCFbased elements converge in a non-monotonic way in the studied cases. The features of the used shape functions are assumed to be responsible for this tendency. As Equation (6.21) shows, the shape func- 
tions are differentiated twice in the calculation of the element curvature which is used in the definition of the elastic forces. The $C^{0}$ continuous shape functions meet the $C^{1}$ continuity requirement only at the nodal points while compatibility conditions at the inter-element edges are not fulfilled. This problem can be rectified by introducing the crossed beam technique which guarantees $C^{1}$ continuity at interelement edges. However, as shown in Fig. 14, $C^{1}$ continuous shape functions do not meet compatibility conditions when used to discretize a curved structure. This means that the kinematics of these thin ANCF shell elements must be improved in order for them to be useful in real life engineering applications.

\section{Improved kinematics of 36 d.o.f. ANCF shell elements using element specific parameters}

In this section, a possible modification of the kinematics of the 36 d.o.f. ANCF shell elements is presented. This is done in order to remedy the problems that have been highlighted in the previous sections. The suggested improvements can also be applied to the 48 d.o.f. element, but the impact of the modification on this element is not studied here. The findings in this section have been published in a conference paper [39] by the author of this thesis and co-authors.

The studied 36 d.o.f. thin ANCF shell elements employ the same order of interpolation as the conventional Q8 element. Therefore, it is believed that improvements can be made to the ANCF elements such that a more accurate representation of curved structures can be obtained. It can be shown that the element configuration and interelement connectivity are sensitive with respect to the element parameters. For instance, changing the length of the slope vectors in the nodes will contribute to the contraction of the element. In the case of the hollow quarter circle, increasing the length of the tangential slope vector, $\frac{\partial \mathrm{r}}{\partial x}$, in nodes 5 and 8 will decrease the contraction of element 3 in the $y$-direction. This will shift the upper edge of this element outward and, at some point, it will match the actual circle it is trying to describe. The increase of the slope vector lengths at nodes 5 and 8 will also affect element 4 . Here, it will increase the contraction of element 4 in the $y$-direction such that the gap between elements 3 and 4 is main- 
13. Improved kinematics of 36 d.o.f. ANCF shell elements using element specific parameters

tained. Hereby, it is concluded that as long as the nodes are shared between curved elements, inter-element connectivity and correct geometrical description cannot be obtained by adjusting the length of the slope vectors at the nodes. Therefore, it is concluded that the kinematics of the thin ANCF shell elements cannot be improved by modifying the nodal coordinates.

Other adjustable element parameters are the physical length and width of the elements. Normally, these are considered constant for an entire element - also in the case of curved elements. As previously mentioned, this assumption is considered to be partly responsible for the loss of inter-element connectivity when elements are used in a curved configuration. In this study, the assumption is relaxed by using the correct lengths and widths of the material strips where the evaluation points on the element are located. This introduces a set of element specific properties that corresponds to the actual initial shape of the element. The number of element specific lengths and widths will depend on the number of desired evaluation points in the element. These element specific lengths and widths will be used whenever the nodal coordinates are interpolated. That being both directly when the shape functions are evaluated (see Equation (3.1)) and in-directly e.g. when calculating the element strains and curvature (see Equations (6.20) and (6.21)). This approach will lead to the problems regarding gaps between curved element sides and the non-constant initial slopes present in the reference configuration being reduced significantly.

The methodology behind the improved kinematics is described in the shell element shown in Fig. 21. The element is shown with sampling points for a three point quadrature rule in both the $x$ and $y$ direction. For each row of sampling points $i$ and $j$, a set of unique

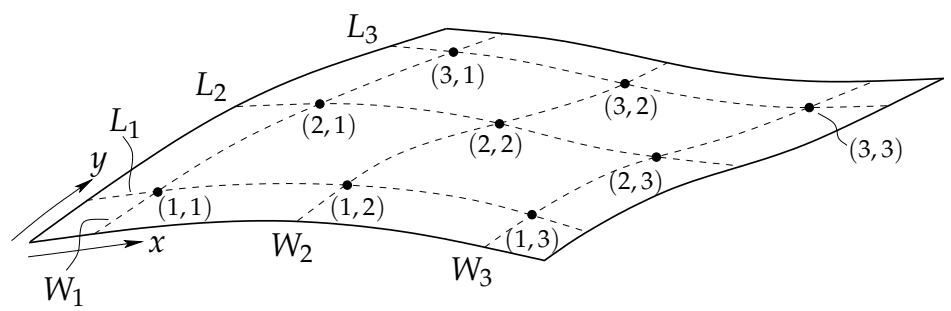

Fig. 21: An ANCF shell element shown with it's respective unique lengths and widths at the sampling points for a third order quadrature rule over the element surface. 
lengths and widths, $L_{i}$ and $W_{j}$, respectively, is introduced. The values of the lengths $L_{i}$ and widths $W_{j}$ correspond to the actual physical lengths and widths of the material strips. These can be calculated in a pre-processing step using e.g. CAD software.

The coordinates of the sampling points $\mathbf{x}(i, j)$ and $\mathbf{y}(i, j)$ are calculated using the unique lengths and widths. In this case where three coordinates in both the $x$ and $y$ direction are needed, these values lie in the intervals:

$$
\begin{aligned}
& 0 \leq \mathbf{x}(i, 1 . .3) \leq L_{i} \\
& 0 \leq \mathbf{y}(1 . .3, j) \leq W_{j}
\end{aligned}
$$

This leads to nine unique values for both the $x$ and $y$ coordinates of the sampling points for arbitrarily shaped elements.

To demonstrate the effect of the improved kinematics, the quarter hollow disc addressed in Section 11 is discretized using ANCF shell elements with the improved kinematic description. This is shown in Fig. 22 together with the same disc discretized by elements using the standard kinematics. Here, modified shape functions based on the crossed beam technique are used, but the same discretization can be obtained using modified shape functions based on the incomplete quartic polynomial. Upon inspection of Fig. 22b, it is clear that the gaps between adjacent curved element sides have vanished and the discretization appears as sound as the one obtained previously using the conventional Q8 elements (see Fig. 15).

Furthermore, by qualitative comparison, it appears as if the outer and inner edges of the disc are more circular in Fig. 22b where the improved kinematics is used than in Fig. 22a. This is even more evident if the edges of elements 3 and 4 between nodes 5 and 8 are compared to the actual circle they are attempting to describe (see Fig. 23a). This comparison shows that the element edges lie on the circle section they are meant to describe. Additionally, it is seen that the evaluation points are equally spaced, which stands in sharp contrast to the results shown in Fig. 16a. Again, the ANCF shell elements using the improved kinematics produce similar figures to those obtained using the Q8 elements (see Fig. 17). The improvement in the ANCF discretization becomes even more clear if the norm of the slopes at the edges between node 5 and 8 obtained using the improved kinematics is compared to the slopes obtained using the standard description (see 
13. Improved kinematics of 36 d.o.f. ANCF shell elements using element specific parameters

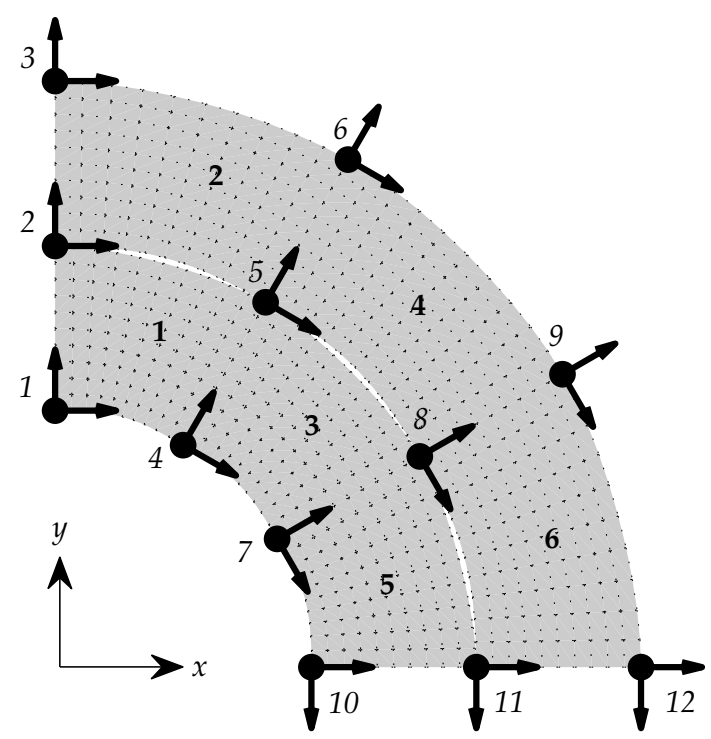

(a) Discretization using standard kinematics.

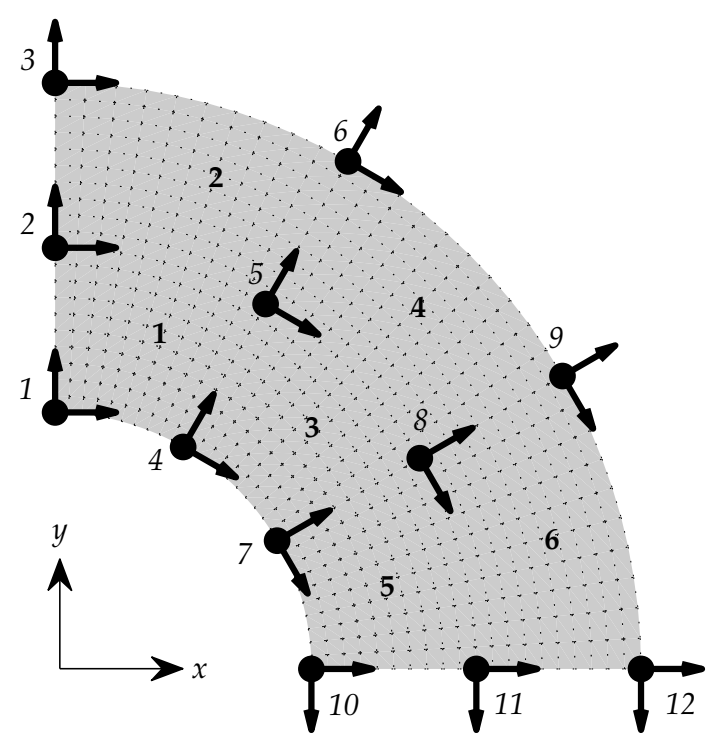

(b) Discretization using improved kinematics.

Fig. 22: A hollow disc with an inner radius of $3.5 \mathrm{~m}$ and an outer radius of $8 \mathrm{~m}$ discretized by 6 thin rectangular ANCF shell elements using two different kinematic descriptions. The nodes are shown with their corresponding slope vectors. Node numbers are given in italic and element numbers are given in bold. 


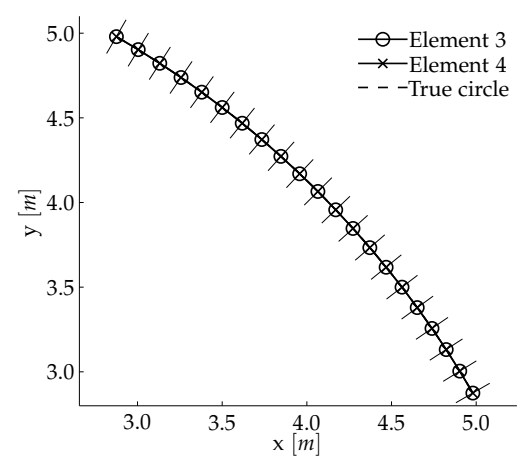

(a) Adjacent element edges for elements 3 and 4 plotted using the improved kinematics together with the geometrically true circle they are attempting to describe.

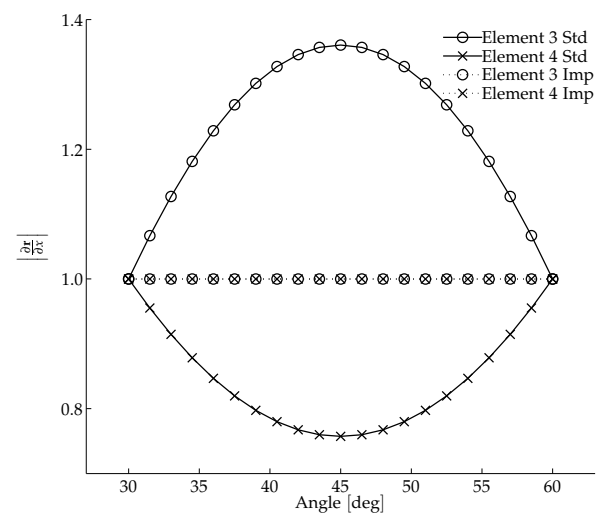

(b) Norms of the slope vectors at the element edges on the interface between elements 3 and 4 .

Fig. 23: Figures demonstrating the influence of applying the improved kinematics.

Fig. 23b). Here, it is seen that there is no variation of the norm of the slope vector along the edge, which is desirable since the slopes at both of the nodes are defined as unit vectors.

The improved kinematics of the thin ANCF shell element have shown that it can lead to a discretization similar to the one produced using Q8 elements. Now, the hope is that the thin ANCF shell elements with the improved kinematics will show better convergence behavior than the ANCF element with standard kinematics. To test this hypothesis, the hollow disc structure as described in Sections 11 and 12 is analyzed again. Results are obtained for both $C^{1}$ and $C^{0}$ continuous shape functions using the improved kinematics, and the results are compared to those obtained using the standard description. These results are shown in Fig. 24.

Based on Fig. 24, it is concluded that the ANCF elements using the improved kinematics still converge in a non-monotonic way in the studied cases. The models using the improved kinematics only show little or practically no difference when compared to the ANCF elements that use the standard description. This is unexpected since it was assumed that if the problems regarding the gaps between the el- 
13. Improved kinematics of 36 d.o.f. ANCF shell elements using element specific parameters

ements in the initial configuration were treated, a better convergence behavior could be obtained. This suggests that the problems regarding the discontinuous displacement field are not fully treated. The improved kinematic description proposed in this study was intended to remedy problems related to compatibility conditions and, consequently, to make the studied ANCF-based elements converge monotonically. That being a convergence tendency as noted in the benchmark study (see Section 9). Unfortunately, this more desirable behavior was not demonstrated. 36 d.o.f. ANCF elements based on either $C^{0}$ or $C^{1}$ continuous shape functions, regardless of the introduced improvements, do not satisfy the compatibility requirement. As a result, 36 d.o.f. ANCF elements do not converge monotonically; a conclusion clearly demonstrating that particular attention must be paid to the convergence of the studied ANCF elements. 

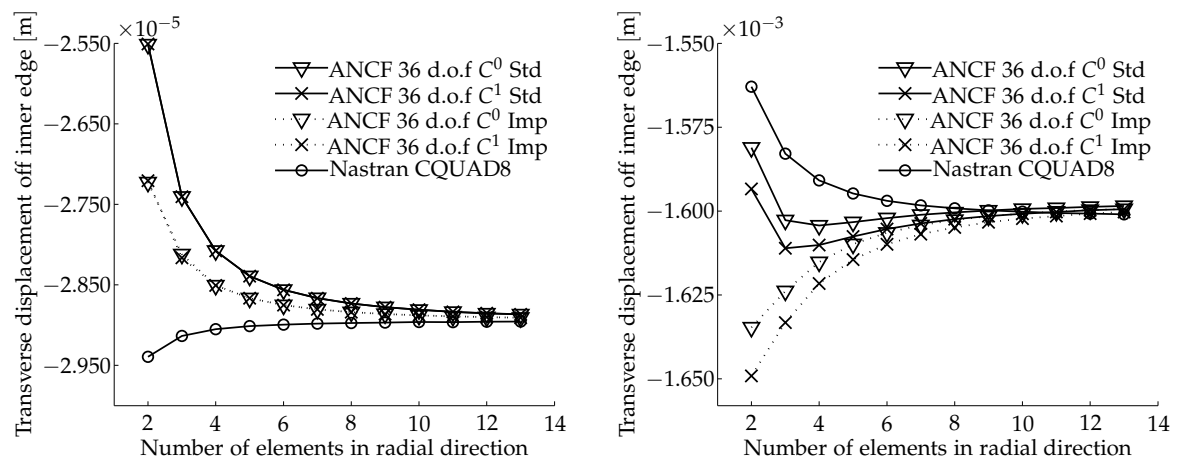

(a) Convergence plot for a total load of $-1 \times 10^{-5} \mathrm{~N}$.

(b) Convergence plot for a total load of $-1 \times 10^{-3} \mathrm{~N}$.
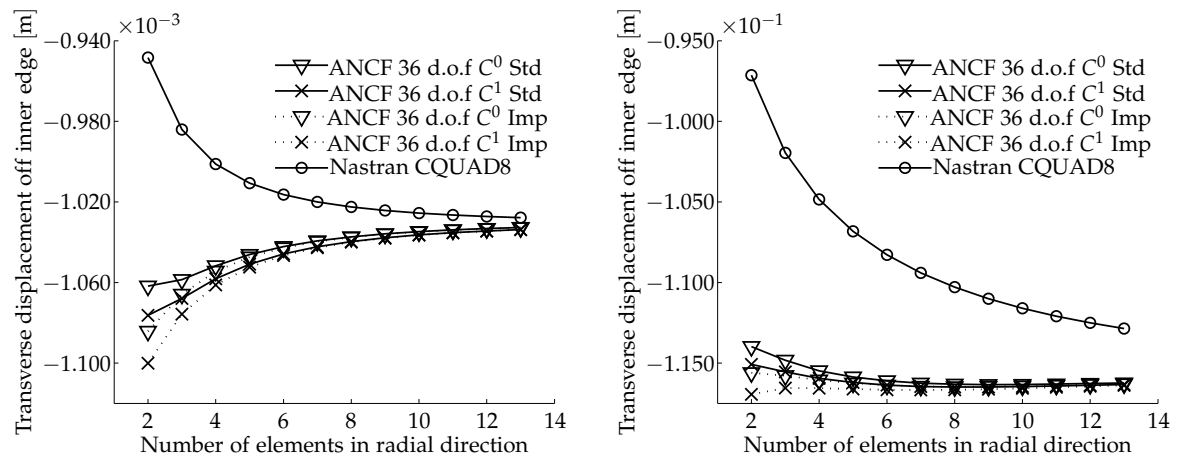

(c) Convergence plot for a total load of (d) Convergence plot for a total load of $-1 \times 10^{-1} \mathrm{~N}$. $-1 \times 10^{2} \mathrm{~N}$.

Fig. 24: Convergence study on a simply supported hollow disc for four different load cases using 36 d.o.f. ANCF shell elements. Both $C^{0}$ and $C^{1}$ continuous shape functions with the standard and improved kinematics are used. 


\section{Interim conclusion}

This comprehensive and thorough investigation has discussed the behavior and performance of gradient deficient thin ANCF shell elements in various scenarios. Initially, the kinematic description of the elements was reviewed (Section 9). Here, the differences in the obtained interpolation shape functions were pointed out. An initial convergence study on a simply supported rectangular plate modeled with an optimal mesh demonstrated the difference in using $C^{0}$ and $C^{1}$ continuous shape functions. Additionally, the simulation results showed that the analyzed ANCF elements obtained converged solutions that were consistently stiffer than reference solutions obtained using a commercial software. This trend prevailed for both the small and the large deflection problems. Furthermore, a significantly higher number of ANCF elements were needed to obtain converged solutions when compared to the reference solution. This indicates problems regarding locking, which has not been alleviated in this study.

The performance of thin ANCF shell elements for analyzing models with non-uniform mesh was studied in Section 10. The study concluded that when an inadequate number of elements is used, the ANCF elements are more sensitive to abrupt changes in element sizes than conventional four and eight node finite elements. However, as the mesh was refined, the ANCF elements showed better performance. A tendency that persisted, though, was that it was difficult to obtain static equilibrium when the models with high element aspect ratio were analyzed. Overall, this small study concluded that thorough convergence studies are necessary when using thin ANCF shell elements in non-uniform meshes.

In Section 11, the ability of the thin ANCF elements to discretize curved structures was discussed. Here, it was noted that the studied quarter hollow disc could not be represented accurately as the discretized model had gaps between adjacent curved element sides. Furthermore, it was noted that the interpolated evaluation points were unevenly spaced, which was linked to a previously noted phenomena called CID. The quarter hollow disc was discretized with conventional eight noded shell elements and compared qualitatively with the ANCF

discretization. Here, it was observed how the curved structure was represented more accurately using the Q8 elements and without gaps 
between elements. This is remarkable since both the ANCF and the Q8 elements use the same order of polynomials in the interpolation of the generalized nodal coordinates. This indicates that it is more favorable to use position d.o.f. than slopes for describing curved structures.

The convergence study in Section 12 was conducted in order to asses whether the discontinuous displacement field had any influence on simulation results. In the initial discussion, consequences such as shape sensitive behavior and abnormal convergence were mentioned, which further manifested the need for such a study. Indeed, the results from the convergence showed that as the applied load on the model was increased, the ANCF models predicted an increasingly large deformation when compared to the reference solution. This stood in contrast to a previously noted behavior based on models using optimal and rectangular ANCF elements. Furthermore, it was noted that increasing the order of quadrature rule for evaluating the internal elastic forces reduced the number of necessary iterations needed in the static solution procedure and gave improved results.

In Section 13, an attempt was made to improve the behavior of the thin ANCF shell elements. In the previous sections, the poor kinematics leading to the incompatible displacement field was assumed to be the source of the inconsistent convergence behavior. To remedy the problems and improve the convergence behavior, a modified kinematic description was introduced. Using this, it was demonstrated that the hollow quarter disc could be discretized to the same accuracy as when using the conventional Q8 element, and that the evaluation points were evenly spaced. However, the desired improvement on the convergence behavior did not appear. The convergence study on the ANCF elements using the improved kinematics indicates that it is some more fundamental issues of the studied elements that are responsible for the undesirable tendencies. That being the non-monotonic convergence behavior when analyzing curved structures and the difficulties in finding static equilibrium.

Overall, the investigation carried out and presented in this part has highlighted problems and issues regarding thin ANCF shell elements. Although these findings can make the ANCF appear erroneous and ineffective, it has not been the intention, whatsoever. In contrast, the goal has been to discuss and emphasize these difficulties such that they can be treated in future studies, leading to a more robust and 
versatile ANCF. In the investigation, the studied cases have been as simple as possible to support the proven points in the best possible manner. These examples have been static analyses of simple structures where both ANCF shell formulations and conventional finite elements are compared. It can be argued that since the ANCF is intended for analysis of large rotation and deformation problems in the multibody dynamics framework, the investigation should also have included such examples. However, it is not believed that the mentioned problems and conclusions will stand out more clearly or be supported in a better way by adding additional studies where large rotation problems in a dynamic time domain simulation are considered. It is believed that ANCF should perform well in both static, small deflection problems as well as in dynamic problems with large rotations and deformations. Such characteristics are necessary to ensure robust and versatile ANCF elements. 
Behavior of thin ANCF shell elements in various mesh configurations 


\section{Part III}

\section{Shear and thickness deformable ANCF shell elements}





\section{Shear and thickness deformable ANCF shell elements}

To this point, two different types of ANCF shell elements has been treated in this thesis. That being the fully parameterized and the thin gradient deficient element. The thin ANCF shell element is only applicable for thin structures where transverse shear deformation can be neglected. Furthermore, as it lacks a local element coordinate in the transverse direction, general constitutive relations cannot be used directly in conjunction with this element. This means that the thin ANCF shell element has restricted usage in fully non-linear structural problems.

The fully parameterized element, on the other hand, is capable of describing both transverse shear and thickness deformation, and general constitutive relations can be included in the calculation of the internal elastic forces when using a continuum mechanics approach (see Equation (6.17)). This widens the area of application significantly. Furthermore, as methods for treating problems regarding locking has been introduced, the fully parameterized element performs well for both thin and thick shell structures [51]. However, fully parameterized elements often have a large number of d.o.f. due to that they employ a full set of slope coordinates (see Fig. 3 and Equation (4.4)), and in some cases also a second order transverse slope vector [51].

Recently, simpler elements with a reduced set of generalized coordinates have been introduced [17]. Here, the inplane slope vectors are omitted from the generalized coordinates, leading to a kinematic description based on the nodal positions and transverse slope vectors. 
This choice of parameterization has also been used for modeling of shear deformable beam elements with success [26]. Using this, the element is still capable of describing transverse shear and thickness deformation, but the total number of element d.o.f. is reduced significantly when compared to fully parameterized elements.

The simpler version of the fully parameterized element is a four noded quadrilateral element with 24 d.o.f using bilinear interpolation. In the originally proposed form [17], selective reduced integration and modified Gaussian integration of the elastic forces were utilized to alleviate inplane shear locking and transverse shear locking, respectively. More recently, the element has been further improved by the use of enhanced assumed strain [81] and assumed natural strain [22, 10]. These means have proved effective for alleviating problems associated inplane shear, transverse shear and thickness locking when using both the elastic midplane approach [92] and the full continuum mechanics approach [98] for deriving the internal elastic forces.

As the four noded shear deformable ANCF shell element is lacking inplane slope vectors, the element utilizes bilinear interpolation of the nodal positions. Due to this, the element will have straight sides, but it is, in general, not restricted to pure square or rectangular shapes and the element can be applied for modeling curved structures. However, a curved edge will be discretized using piecewise linear segments and several elements are necessary for satisfactory representation of curved and arbitrary shaped structures.

In this part, a quadratic shear and thickness deformable element is introduced. Such an element will be an supplement to the bilinear element for enhanced modeling curved structures. The aim is to build upon the experience gained during the development of the bilinear shear deformable element and formulate an efficient and locking-free quadratic element. Here, already tested and verified methods for alleviating locking mechanisms in conventional quadratic shell elements will be applied. The stiffness description is based on an elastic midplane approach where the internal elastic energy of the element is calculated as the sum of four characteristic strain energy states. This approach reduces coupling between the different strain states, and thus removes some undesirable locking mechanisms in an early stage.

Conclusively, this part is ended with a discussion on similarities between the shear deformable ANCF shell elements and similar tradi- 
15. Parameterization of quadratic shear and thickness deformable ANCF shell element

tional finite elements. This is included in order to further predict the performance of the developed quadratic ANCF shell element.

The development of the quadratic element is done in collaboration with Professor Sugiyama, University of Iowa, and has been initiated during a visit at his research group.

\section{Parameterization of quadratic shear and thick- ness deformable ANCF shell element}

Prior to the actual formulation of the quadratic element some very basic but important decisions must be made. These choices concern the parameterization of the element. As in the case of the bilinear shear deformable element, the quadratic element will be formulated without using inplane slope vectors. Thus, in order for the quadratic element to have curved element sides additional nodes are placed on the midsides of the element. This gives some benefits when compared to using inplane slope vectors for describing curved shapes. To demonstrate this, two circular line segments are shown in Fig. 25.

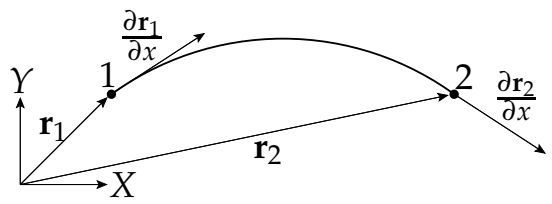

(a) Representation using slopes.

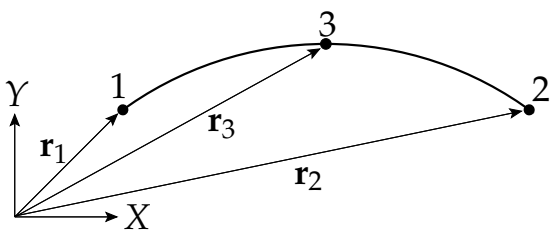

(b) Representation using midpoint node.

Fig. 25: Representation of circle segment by quadratic interpolation using two different parameterizations.

In Fig. 25a the circle segment is represented using two nodes and two slope vectors at the end points of the line. In Fig. 25b, the same segment is discretized, but here one additional node located at the middle of the segment is used instead of the two slope vectors. The two parameterizations will both use the same quadratic interpolation, but the one using position d.o.f. (Fig. 25b) will only use three vectors as generalized coordinates, whereas the other (Fig. 25a) will use four. This demonstrates that a curved line segment can be represented using three less d.o.f. when using a node on the element midside instead 
of slope vectors at the endpoint nodes. Additionally, as discussed in Sections 11 and 13, the use of slope vectors may not be optimal for representation of curved structures.

One downside of not using inplane slope vectors as generalized coordinates is that inter-element slope continuity at the midplane is not ensured. However, similar elements missing this feature are widely used in the conventional finite element method. As long as the lack of inter-element slope continuity is kept in mind when formulating the element this is not considered to be a problem.

The last decision concerns the number of nodes used in the element formulation. Two different families of quadratic shell elements exist in the finite element literature. Those being the eight noded serendipity elements and the nine noded Lagrange elements [14]. The two different elements are shown in Fig. 26 where it is seen how the nodes are located in the elements. The benefits and drawbacks of both elements have been discussed thoroughly in the literature, e.g. $[48,49,101]$. The 9 noded Lagrange element has the advantage of being less sensitive to the position of the midside nodes, and it passes the out-of-plane bending patch test when the sides are curved. The 8 noded serendipity element can loose accuracy when the midside node is located far away from the center of the midside and the element must have straight sides in order to pass the out-of-plane bending patch test. This, of course, favors the nine noded topology. On the other hand, both element types are known to suffer from extensive locking problems, and

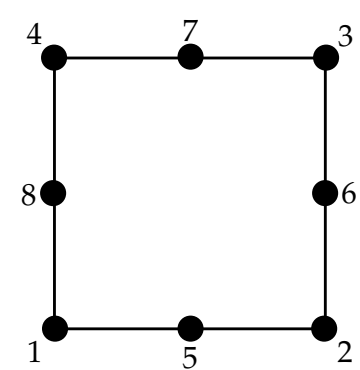

(a) Eight noded serendipity element.

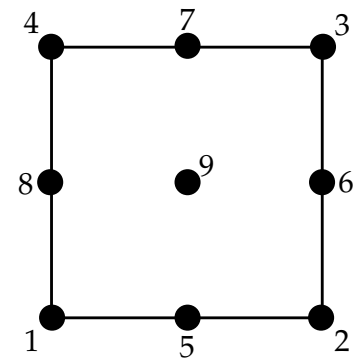

(b) Nine noded Lagrange element.

Fig. 26: Two quadratic shell finite elements with different number of nodes. 
16. Kinematics of a quadratic shear and thickness deformable ANCF shell element

the most simple methods to address these locking mechanisms exists for the eight noded element. Furthermore, the extra node in the nine node element leads to a higher number of degrees of freedom. Due to the simpler topology and the prospect of less complicated methods for alleviating locking, it is chosen to base the quadratic shear deformable ANCF shell element on the eight noded serendipity element topology.

\section{Kinematics of a quadratic shear and thickness deformable ANCF shell element}

The eight noded shear and thickness deformable element is shown in Fig. $27^{3}$. The midplane of the element is defined entirely by the area spanned by the nodes and the orientation and deformation of the cross section is described by the transverse slope vectors. This leads to a kinematic description where the element is described as a volume. Additionally, as the transverse slope vector is not kinematically linked to the element midplane, the element is capable of describing transverse shear deformation.

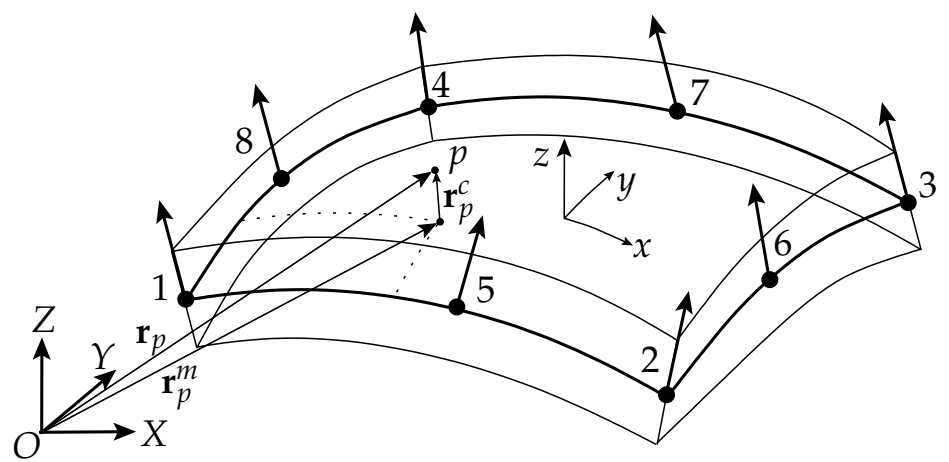

Fig. 27: Eight noded quadratic shear and thickness deformable ANCF shell element shown with nodal numbers. Position and slope vectors are not shown for brevity.

A point $p$ in the volume is described by a position vector $\mathbf{r}_{p}$ expressed with respect to the global reference frame $O X Y Z$ which can be calculated as follows:

$$
\mathbf{r}_{p}=\mathbf{r}_{p}^{m}+\mathbf{r}_{p}^{c} .
$$

${ }^{3}$ Note that a similar element has been mentioned recently [62], but not studied in detail. 
Here, $\mathbf{r}_{p}^{m}$ is a vector describing the projection of point $p$ along the cross section on the element midplane and $\mathbf{r}_{p}^{c}$ is a vector oriented along the cross section from the midplane to point $p$. The vectors $\mathbf{r}_{p}^{m}$ and $\mathbf{r}_{p}^{c}$ are expressed by interpolation of the nodal position and slope vectors, which leads to the following expression for the vector $\mathbf{r}^{p}$ :

$$
\mathbf{r}_{p}=\mathbf{N}\left(x_{p}, y_{p}\right) \cdot \mathbf{q}_{r}+z_{p} \cdot \mathbf{N}\left(x_{p}, y_{p}\right) \cdot \mathbf{q}_{s} .
$$

Here, $x_{p}, y_{p}, z_{p}$ are the local element coordinates of point $p$ and $\mathbf{q}_{r}$ and $\mathbf{q}_{s}$ are vectors containing the position and slope vector coordinates, respectively. The nodal d.o.f. are assembled in the vector of generalized coordinates q:

$$
\begin{aligned}
& \mathbf{q}=\left[\begin{array}{ll}
\left(\mathbf{q}_{r}\right)^{T} & \left(\mathbf{q}_{s}\right)^{T}
\end{array}\right]^{T} \\
& \mathbf{q}_{r}=\left[\begin{array}{lllll}
\mathbf{r}_{1}^{T} & \mathbf{r}_{2}^{T} & \ldots & \mathbf{r}_{7}^{T} & \mathbf{r}_{8}^{T}
\end{array}\right]^{T} \\
& \mathbf{q}_{s}=\left[\left(\frac{\partial \mathbf{r}_{1}}{\partial z}\right)^{T}\left(\frac{\partial \mathbf{r}_{2}}{\partial z}\right)^{T} \ldots\left(\frac{\partial \mathbf{r}_{7}}{\partial z}\right)^{T}\left(\frac{\partial \mathbf{r}_{8}}{\partial z}\right)^{T}\right]^{T}
\end{aligned}
$$

which gives the element a total of 48 d.o.f. It should be noted in Equation (16.44) how the position and the slope coordinates are interpolated using the same shape function matrix, N. Furthermore, the last term is multiplied by the local transverse coordinate $z$ to give the variation in the transverse direction. The coordinate $z$ is defined in the range $-h / 2 \leq z \leq h / 2$, where $h$ is the element thickness.

Following the derivation of the classical serendipity element [14], it is assumed that an arbitrary point in the element volume can be described using a complete quadratic polynomial in $x$ and $y$ and the additional cubic terms $x^{2} y$ and $x y^{2}$. This combined polynomial will be used for interpolation of both the position and slope coordinates as stated in Equation (16.44). This leads to that an arbitrary point in the element volume is described by:

$$
\begin{aligned}
\mathbf{r}= & \mathbf{a}_{1}+\mathbf{a}_{2} x+\mathbf{a}_{3} y+\mathbf{a}_{4} x^{2}+\mathbf{a}_{5} x y+\mathbf{a}_{6} y^{2}+\mathbf{a}_{7} x^{2} y+\mathbf{a}_{8} x y^{2}+ \\
& z \cdot\left(\mathbf{a}_{9}+\mathbf{a}_{10} x+\mathbf{a}_{11} y+\mathbf{a}_{12} x^{2}+\mathbf{a}_{13} x y+\mathbf{a}_{14} y^{2}+\mathbf{a}_{15} x^{2} y+\mathbf{a}_{16} x y^{2}\right)
\end{aligned}
$$

For both polynomials, $\mathbf{a}_{\mathbf{i}}$ are $3 \times 1$ vectors of polynomial coefficients that depend on the generalized nodal coordinates. Using the polynomial in Equation (16.46), eight individual shape functions can be 
derived and the shape function matrix $\mathbf{N}$ takes the form:

$$
\mathbf{N}(\xi, \eta)=\left[\begin{array}{lllll}
N_{1}(\xi, \eta) & N_{2}(\xi, \eta) & \ldots & N_{7}(\xi, \eta) & N_{8}(\xi, \eta)
\end{array}\right] \otimes \mathbf{I}
$$

where the eight individual shape functions $N_{i}$ are given by:

$$
\begin{aligned}
& N_{1}=-\frac{1}{4}(\xi-1)(\eta-1)(\eta+\xi+1) \\
& N_{2}=\frac{1}{4}(\xi+1)(\eta-1)(\eta-\xi+1) \\
& N_{3}=\frac{1}{4}(\xi+1)(\eta+1)(\eta+\xi-1) \\
& N_{4}=-\frac{1}{4}(\xi-1)(\eta+1)(\eta-\xi-1) \\
& N_{5}=\frac{1}{2}(\xi-1)(\xi+1)(\eta-1) \\
& N_{6}=-\frac{1}{2}(\eta-1)(\eta+1)(\xi+1) \\
& N_{7}=-\frac{1}{2}(\xi-1)(\xi+1)(\eta+1) \\
& N_{8}=\frac{1}{2}(\eta-1)(\eta+1)(\xi-1)
\end{aligned}
$$

Here, normalized element coordinates $\xi$ and $\eta$ given by $\xi=\frac{x}{2 L}$ and $\eta=\frac{y}{2 W}$ have been used in which $L$ and $W$ are the length and width of the element, respectively. The coordinates $\xi$ and $\eta$ take values in the range $-1 \leq \xi, \eta \leq 1$.

It should be emphasized that the cross section is described entirely by the transverse gradient vector and the midplane by the nodal positions. This means that no kinematic assumptions are introduced regarding the cross section orientation and deformation with respect to the element midplane, which allows for both transverse shear and thickness deformation.

\section{Elastic forces}

The equations of motion for the quadratic ANCF shell element can be derived by following the procedure given in Section 6 . The calculation of the vector of internal elastic forces, however, takes a different form as the shear and thickness deformation must be taken into account. 
In this study, the internal elastic forces are evaluated using the elastic midplane approach where the internal elastic energy is divided into parts based on the possible deformations of the element. In this case, the elastic energy is divided into:

- Inplane normal and shear deformation of the midplane.

- Transverse shear deformation.

- Thickness deformation.

- Bending and twisting of the midplane.

By summing op the energy carried in each of these deformation modes, the total internal energy is expressed as:

$$
\begin{aligned}
W_{e}=\frac{h}{2} \int_{A_{0}} \boldsymbol{\varepsilon}_{m}^{T} \mathbf{D}_{m} \boldsymbol{\varepsilon}_{m} \mathrm{~d} A+ & \frac{h}{2} \int_{A_{0}} \gamma_{t}^{T} \mathbf{D}_{\gamma} \gamma_{t} \mathrm{~d} A+ \\
& \frac{h}{2} \int_{A_{0}} \varepsilon_{z z} E \varepsilon_{z z} \mathrm{~d} A+\frac{1}{2} \int_{A_{0}} \boldsymbol{\kappa}^{T} \mathbf{D}_{\kappa} \kappa \mathrm{d} A
\end{aligned}
$$

Inserting Equation (17.49) into Equation (6.11), the vector of internal elastic forces is calculated as:

$$
\begin{array}{r}
\mathbf{Q}_{e}=h \int_{A_{0}}\left(\frac{\partial \boldsymbol{\varepsilon}_{m}}{\partial \mathbf{q}}\right)^{T} \mathbf{D}_{m} \boldsymbol{\varepsilon}_{m} \mathrm{~d} A+h \int_{A_{0}}\left(\frac{\partial \gamma_{t}}{\partial \mathbf{q}}\right)^{T} \mathbf{D}_{\gamma} \gamma_{t} \mathrm{~d} A+ \\
h \int_{A_{0}}\left(\frac{\partial \varepsilon_{z z}}{\partial \mathbf{q}}\right)^{T} E \varepsilon_{z z} \mathrm{~d} A+\int_{A_{0}} \delta\left(\frac{\partial \boldsymbol{\kappa}}{\partial \mathbf{q}}\right)^{T} \mathbf{D}_{\kappa} \kappa \mathrm{d} A
\end{array}
$$

Here, $\varepsilon_{m}$ is a vector containing the in-plane normal and shear strains, $\gamma_{t}$ is a vector containing the transverse shear strains, $\varepsilon_{z z}$ is the transverse normal strain, and $\kappa$ is a vector containing the midplane curvature components. $\mathbf{D}_{m}, \mathbf{D}_{\gamma}$ and $\mathbf{D}_{\kappa}$ are constitutive matrices and $E$ is the Young's modulus. $h$ is the thickness of the element and $A_{0}$ is the element area in the initial state.

The inplane, the transverse shear and the transverse normal strains are all calculated using the Green-Lagrange strain tensor (see Equation (6.15)). In addition, the strain and curvature components are evaluated at the element midplane, hence $z=0$. Using this, the inplane strains, 
17. Elastic forces

$\mathcal{\varepsilon}_{m}$, are given by:

$$
\begin{aligned}
& \varepsilon_{m}=\left[\begin{array}{lll}
\varepsilon_{x x} & \varepsilon_{y y} & 2 \varepsilon_{x y}
\end{array}\right]^{T} \\
& \varepsilon_{x x}(x, y, 0)=\frac{1}{2}\left(\left(\frac{\partial \mathbf{r}}{\partial x}\right)^{T}\left(\frac{\partial \mathbf{r}}{\partial x}\right)-1\right) \\
& \varepsilon_{y y}(x, y, 0)=\frac{1}{2}\left(\left(\frac{\partial \mathbf{r}}{\partial y}\right)^{T}\left(\frac{\partial \mathbf{r}}{\partial y}\right)-1\right) \\
& \varepsilon_{x y}(x, y, 0)=\frac{1}{2}\left(\left(\frac{\partial \mathbf{r}}{\partial x}\right)^{T}\left(\frac{\partial \mathbf{r}}{\partial y}\right)\right),
\end{aligned}
$$

the transverse shear strains are given by:

$$
\begin{aligned}
& \gamma_{t}=\left[\begin{array}{ll}
\gamma_{x z} & \gamma_{y z}
\end{array}\right]^{T} \\
& \gamma_{x z}(x, y, 0)=\left(\frac{\partial \mathbf{r}}{\partial x}\right)^{T}\left(\frac{\partial \mathbf{r}}{\partial z}\right) \\
& \gamma_{y z}(x, y, 0)=\left(\frac{\partial \mathbf{r}}{\partial y}\right)^{T}\left(\frac{\partial \mathbf{r}}{\partial z}\right),
\end{aligned}
$$

and the transverse normal strain is given by:

$$
\varepsilon_{z z}(x, y, 0)=\frac{1}{2}\left(\left(\frac{\partial \mathbf{r}}{\partial z}\right)^{T}\left(\frac{\partial \mathbf{r}}{\partial z}\right)-1\right)
$$

The curvatures of the element are given by [17]:

$$
\begin{aligned}
& \kappa=\left[\begin{array}{lll}
\kappa_{x x} & \kappa_{y y} & 2 \kappa_{x y}
\end{array}\right]^{T} \\
& \kappa_{x x}(x, y, 0)=-\left(\frac{\partial}{\partial x} \frac{\partial \mathbf{r}}{\partial z}\right)^{T} \frac{\partial \mathbf{r}}{\partial x} \\
& \kappa_{y y}(x, y, 0)=-\left(\frac{\partial}{\partial y} \frac{\partial \mathbf{r}}{\partial z}\right)^{T} \frac{\partial \mathbf{r}}{\partial y} \\
& \kappa_{x y}(x, y, 0)=-\left(\frac{\partial}{\partial x} \frac{\partial \mathbf{r}}{\partial z}\right)^{T} \frac{\partial \mathbf{r}}{\partial y}-\left(\frac{\partial}{\partial y} \frac{\partial \mathbf{r}}{\partial z}\right)^{T} \frac{\partial \mathbf{r}}{\partial x}
\end{aligned}
$$

In the calculation of the curvature it is assumed that the midplane normal vector, $\mathbf{n}$, is approximately equal to the transverse slope vector: $\mathbf{n} \cong \frac{\partial \mathbf{r}}{\partial z}$, which is acceptable for moderately stiff materials. 
It should be noted that the calculation of the curvature for the shear and thickness deformable element takes a different approach than the one used for the thin ANCF shell element (see Equation (6.21)). For that element, the curvature is calculated using second order derivatives of the position field. As mentioned earlier, $C^{1}$ continuous interpolation is recommended when the element curvature is calculated using higher order derivatives [101, 29]. However, the interpolation used for the quadratic shear and thickness deformable elements ensures only $C^{0}$ continuous interpolation, which is the reason that this different approach is chosen. Here, the curvature is calculated using the first order derivatives of the transverse slope vector instead. Additionally, the expression for the curvature is simplified significantly compared to the one used for the thin ANCF shell element. Furthermore, by avoiding the use of second order derivatives in the calculation of the element curvature, the curvature may be predicted to a higher level of accuracy [6], but the influence of this will not be treated in this study.

The constitutive matrices used in Equations (17.51)-(17.54) are given by:

$$
\begin{aligned}
& \mathbf{D}_{m}=\frac{E}{1-v^{2}}\left[\begin{array}{ccc}
1 & v & 0 \\
v & 1 & 0 \\
0 & 0 & \frac{1-v}{2}
\end{array}\right] \\
& \mathbf{D}_{\gamma}=\frac{E}{2(1+v)}\left[\begin{array}{cc}
c_{x z} & 0 \\
0 & c_{y z}
\end{array}\right] \\
& \mathbf{D}_{\kappa}=\frac{h^{3}}{12} \mathbf{D}_{m}
\end{aligned}
$$

where $v$ is Poisson's ratio and $c_{x z}$ and $c_{y z}$ are shear correction factors. The shear correction factors are introduced to account for parabolic variation of the transverse shear deformation across the element thickness. The shear factors take the values $c_{x z}=c_{y z}=5 / 6$ for rectangular cross sections [14]. 


\section{Remedies for alleviating locking}

The shear and thickness deformable ANCF shell element will suffer from severe locking mechanisms if the elastic force vector is implemented directly as described in the previous section. Because the problems regarding locking in traditional finite elements are well-known, a substantial amount of research have been done to develop and test methods that can be used to efficiently alleviate or circumvent locking problems $[44,100,90]$. The choice of using the elastic midplane approach solves some locking problems directly, since locking arising due to coupling of the different deformation modes, e.g. Poisson's thickness locking, is avoided. However, the use of the elastic midplane approach does not address all locking problems, hence additional means must be applied to have a locking free element. The following subsections contain a brief review of the methods used to address locking problems in the quadratic shear deformable ANCF shell element.

\subsection{Reduced integration}

One of the earliest methods applied to address locking in quadratic shell elements is the concept of reduced integration (RI). RI have been applied with great success to circumvent poor numerical performance associated to membrane and transverse shear locking in e.g. eight and nine noded shell elements $[102,64,48]$. The use of RI is made possible since practically all finite elements utilize numerical integration for the evaluation of element matrices and vectors. It can be shown that a significant improvement in accuracy and reduction in computational cost can be obtained by modification of the numerical integration. Here, the theoretically necessary order of integration is reduced by one when evaluating terms in the stiffness matrix and elastic force vector associated to the inplane and transverse shear deformation. Thus the term reduced integration. This means that for a quadratic shell element, the theoretically exact $3 \times 3$ sampling points should be substituted with a $2 \times 2$ integration scheme. The explanation for this is that the compatible strain field derived from the interpolation of the nodal displacements predicts the most accurate strains at certain locations. These locations happen to be the $2 \times 2$ sampling points for a Gaussian nu- 
merical integration scheme [5]. Besides the improvement in accuracy, a significant reduction in computational time can be expected since fewer evaluations of the integrands are needed due to the fewer sampling points.

A disadvantage of using reduced integration is that it can lead to spurious mechanisms in certain cases [101]. This applies e.g. for elements with the eight noded topology and interpolation as chosen for the quadratic ANCF element treated here. For this particular element type, a spurious mechanism show up as a seventh zero energy mode. This mode is, however, non-communicating and is suppressed when more than one element is assembled in a finite element mesh and is in general not an issue [101,48]. Elements with other topology and interpolation, on the other hand, may suffer from these spurious mechanisms, which in some cases can lead to models that does not give convergent results. An example of such is the nine noded Lagrange element which is one of the reasons why this element topology was not chosen for the quadratic shear deformable ANCF shell element. However, methods to suppress these spurious mechanisms in nine noded elements have been developed [9], but this will further complicate the use of reduced integration. This makes the use of the nine noded topology unfavorable compared to the eight noded where reduced integration is applicable without further modification.

\subsection{Assumed natural strain}

When using RI, advantage is taken of that that finite elements are capable of predicting theoretically correct strain or stress states at certain locations. This ability is the basis of another widely applied method for addressing locking problems in finite elements, namely the concept of assumed natural strain (ANS). When using the ANS approach, components of the Green-Lagrange strain tensor (see Equations (17.51) - (17.53)) are evaluated at certain points where the strains are assumed to be correct. These strains are then used to span an assumed strain field by interpolation of the sampled strains. Depending on the specific ANS approach, the number of strain sampling points and the interpolation scheme can vary for each of the strain components. When the redistributed strain fields have been calculated, these new strain fields are used in the evaluation the elastic force vector. 
The ANS method have been applied with success in the bi-linear shear deformable ANCF shell element [98, 92]. Here, methods developed for similar traditional shell finite elements are applied to alleviate two different locking mechanisms. Transverse shear locking is treated using a 2 point sampling method with linear interpolation [22], and thickness locking is addressed using 4 point sampling with bilinear interpolation [10]. For the quadratic ANCF element, ANS methods developed for other quadratic shell elements has been sought.

Two ANS methods for addressing transverse shear locking in eight noded shell elements have been studied. The first method was developed for use in the MITC8 element [22]. Here, each of the transverse shear strain components is sampled at six points and redistributed using quadratic interpolation functions. The six sampling points are shown in Fig. 28a. Note that different points are used for the sampling of $\gamma_{x z}$ and $\gamma_{y z}$ and that the value for $\gamma^{5}$ is obtained as the mean value of the strains sampled at point $5 a$ and $5 b$.

The second method [65] uses similar sampling points, but it evaluates the transverse shear strains directly in the middle of the element instead of using the mean value of two points as shown in Fig. 28b.

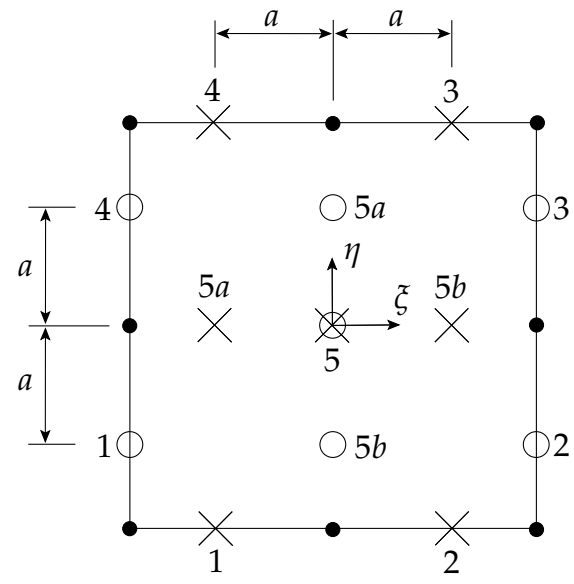

(a) Sampling points for ANS method $I$.

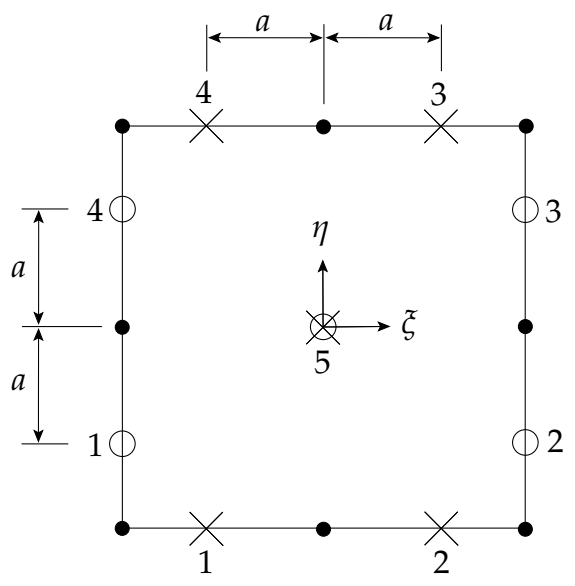

(b) Sampling points for ANS method II.

Fig. 28: Eight noded shell elements shown with sampling points for the ANS methods applied to address transverse shear locking. $\times$ sampling points for $\gamma_{x z}$. $\circ$ sampling points for $\gamma_{y z}$. The distance $a=1 / \sqrt{3}$. 
The sampled values are interpolated using functions that are quadratic in the $\eta$-direction and linear in the $\xi$-direction for $\gamma_{x z}$ and vice versa for $\gamma_{y z}$. Both methods have been implemented and tested and numerical studies have shown that the six point sampling method [22] (see Fig. 28a) showed better performance. Due to this, the six point ANS method will be used in the following studies. Here, the ANS method will be compared to the reduced integration in order to determine which method is the most effective for addressing transverse shear locking.

Using the sampling points as shown in Fig 28a, the redistributed strain fields are calculated as [22]:

$$
\begin{aligned}
& \gamma_{x z}^{A N S}=\sum_{i=1}^{4} h_{x z}^{i} \gamma_{x z}^{i}+h_{x z}^{5} \cdot \frac{1}{2}\left(\gamma_{x z}^{5 a}+\gamma_{x z}^{5 b}\right) \\
& \gamma_{y z}^{A N S}=\sum_{i=1}^{4} h_{y z}^{i} \gamma_{y z}^{i}+h_{y z}^{5} \cdot \frac{1}{2}\left(\gamma_{y z}^{5 a}+\gamma_{y z}^{5 b}\right)
\end{aligned}
$$

with the quadratic interpolation functions $h_{x z}^{i}$ given by:

$$
\begin{aligned}
& h_{x z}^{1}=\frac{1}{4}\left(1-\frac{\xi}{a}\right)(1-\eta)-\frac{1}{4} h_{x z}^{5} \\
& h_{x z}^{2}=\frac{1}{4}\left(1+\frac{\xi}{a}\right)(1-\eta)-\frac{1}{4} h_{x z}^{5} \\
& h_{x z}^{3}=\frac{1}{4}\left(1+\frac{\xi}{a}\right)(1+\eta)-\frac{1}{4} h_{x z}^{5} \\
& h_{x z}^{4}=\frac{1}{4}\left(1-\frac{\xi}{a}\right)(1+\eta)-\frac{1}{4} h_{x z}^{5} \\
& h_{x z}^{5}=\left(1-\left(\frac{\xi}{a}\right)^{2}\right)\left(1-\eta^{2}\right)
\end{aligned}
$$

The interpolation functions $h_{y z}^{i}$ are obtained by substituting $\xi$ with $\eta$ and vice versa in Equation (18.57).

\subsection{Enhanced assumed strain}

The third and last method reviewed in this study to address locking is the enhanced assumed strain (EAS) approach [81]. At first, the EAS method is briefly reviewed in general terms. After this, the specific 
18. Remedies for alleviating locking

implementation that is suggested for the quadratic ANCF shell element is introduced.

When using the EAS an additional term is added to a strain component to enhance the elements capability to represent that strain. The enhanced strain is added as follows:

$$
\tilde{\varepsilon}=\varepsilon^{G L}+\varepsilon^{E A S}
$$

where $\varepsilon^{G L}$ is the Green-Lagrange strain derived from the displacement field and $\varepsilon^{E A S}$ is the enhanced strain. The enhanced strain component $\varepsilon^{E A S}$ is calculated as a product of a set of assumed strain polynomials $\mathrm{G}$ and a unknown vector of polynomial coefficients $\alpha$ :

$$
\varepsilon^{E A S}=\mathbf{G} \cdot \boldsymbol{\alpha}
$$

where $\mathbf{G}$ is defined in terms of the local element coordinates $x$ and $y$. A coordinate transformation must be applied in cases where an element is not initially flat and aligned with the global reference frame [81]. The polynomials used to define the matrix $\mathbf{G}$ cannot be chosen arbitrarily if the element should pass the patch test which is necessary to ensure convergent results. It can be shown that if the following equation is fulfilled, the patch test will be passed [81]:

$$
\int_{V} \mathbf{G} \mathrm{d} V=0
$$

The EAS parameter vector $\alpha$ is a state variable that will vary depending on the strain state in the element and must be updated at each iteration during the solution process. The calculation of the EAS parameters are done separately from the calculation of the nodal coordinates using a static condensation. The vector of EAS parameters $\alpha$ at iteration $n+1$ is given by:

$$
\boldsymbol{\alpha}^{(n+1)}=\boldsymbol{\alpha}^{(n)}-\left(\mathbf{H}^{(n)}\right)^{-1}\left(\boldsymbol{\Gamma}^{(n)} \Delta \mathbf{q}^{(n)}-\mathbf{h}^{(n)}\right)
$$

where $\Delta \mathbf{q}^{(n)}$ is the vector used to update the nodal coordinates:

$$
\mathbf{q}^{(n+1)}=\mathbf{q}^{(n)}+\Delta \mathbf{q}^{(n)}
$$


$\mathbf{H}, \boldsymbol{\Gamma}$, and $\mathbf{h}$ are element specific matrices given by:

$$
\begin{aligned}
\mathbf{H} & =\int_{V_{0}} \mathbf{G}^{T} \mathbf{D} \mathbf{G} \mathrm{d} V \\
\boldsymbol{\Gamma} & =\int_{V_{0}} \mathbf{G}^{T} \mathbf{D} \frac{\partial \boldsymbol{\varepsilon}}{\partial \mathbf{q}} \mathrm{d} V \\
\mathbf{h} & =\int_{V_{0}} \mathbf{G}^{T} \mathbf{D} \tilde{\varepsilon} \mathrm{d} V
\end{aligned}
$$

Here, $\mathbf{D}$ is the constitutive relation associated to the particular GreenLagrange strain which is enhanced. Note that the $\frac{\partial \varepsilon}{\partial q}$ in the calculation of $\boldsymbol{\Gamma}$ is just the partial derivative of the normal Green-Lagrange strains with respect to the element nodal coordinates. This is due to the fact that the enhanced strain $\varepsilon^{E A S}$ vanishes in the differentiation since it has no explicit dependency of the nodal coordinates.

Beside the contribution to the strain tensor, additional contributions must be added to the vector of elastic forces $\mathbf{Q}_{e}$ and the tangent stiffness matrix $\mathbf{K}_{e}$ due to the static condensation of the EAS parameters. The enhanced components $\tilde{\mathbf{Q}}_{e}$ and $\tilde{\mathbf{K}}_{e}$ are given by:

$$
\begin{aligned}
\tilde{\mathbf{Q}}_{e} & =\mathbf{Q}_{e}-\Gamma^{T}\left(\mathbf{H}^{-1} \mathbf{h}\right) \\
\tilde{\mathbf{K}}_{T} & =\mathbf{K}_{T}-\Gamma^{T}\left(\mathbf{H}^{-1} \boldsymbol{\Gamma}\right)
\end{aligned}
$$

After these updated matrices are calculated for each element, the total elastic force vector and tangent stiffness matrix for the entire structure is assembled such that an updated set of nodal coordinates, $\mathbf{q}^{(i+1)}$, and EAS parameters, $\boldsymbol{\alpha}^{(i+1)}$, can be calculated and the next iteration step is performed. It is important to note that matrices $\mathbf{H}, \boldsymbol{\Gamma}$, and $\mathbf{h}$ for each element must be stored for the next iteration, since they are needed to update the EAS parameters $\alpha$ (see Equation (18.61)).

The EAS has previously been used to address problems of inplane shear and thickness locking in several bi-linear elements, e.g. [3, 10, 95, $98,92]$, and is an essential part of their respective stiffness description. In the case of quadratic elements, however, the use of EAS have not been as widespread. One application has been to use EAS to address thickness locking in a quadratic solid-shell element with nine nodes [32]. Here the transverse normal strain, $\varepsilon_{z z}$, is enhanced using a nine parameter EAS polynomial as follows:

$$
\tilde{\varepsilon}_{z z}=\varepsilon_{z z}+\mathrm{G} \cdot \alpha
$$


where $\varepsilon_{z z}$ is defined in Equation (17.53) and the matrix of EAS polynomials, $\mathbf{G}$, is given by:

$$
\mathbf{G}=z \cdot\left[\begin{array}{lllllllll}
1 & x & y & x y & x^{2} & y^{2} & x^{2} y & x y^{2} & x^{2} y^{2}
\end{array}\right]
$$

Here $z$ is the local transverse coordinate. By applying this strain enhancement, $\varepsilon_{z z}$ will be able to vary over the cross section, and its contribution to the vector of elastic forces must be evaluated using a volume integral.

In this study, it is not expected that the EAS will enhance the performance of the quadratic ANCF shell element. This is due to the fact that the elastic midplane approach is applied to derive the vector of elastic forces. Here, the inplane and transverse normal strains are decoupled which will alleviate the Poisson's thickness locking [92]. However, in the case where the continuum mechanics approach is applied for deriving the vector of elastic forces, the use of EAS could prove to be crucial to ensure a well performing element.

\section{Numerical examples}

This section contains some preliminary numerical results to give indications of the performance of the novel quadratic shear and thickness deformable ANCF element. The element will be tested using static and eigenfrequency analyses which will be compared to results obtained using both analytical models and commercial software. In addition to testing the overall performance of the new element, the numerical studies will be used to assess which combinations of locking remedies that are the most effective.

The locking remedies will be applied and analyzed in stages to asses the influence and performance of the different locking remedies individually. The different combinations will be referred to using the notation described in Table 3. For all the models, the calculation of the curvature is implemented directly as in Equation (17.54) without modifications. As default the terms in the elastic force vector is integrated using full $3 \times 3$ Gaussian integration. However, when the reduced integration is applied, the order of integration is reduced to a $2 \times 2$ scheme for the affected terms. 
Name

Description

ANCF Unmodified

The quadratic element implemented directly using the calculation of the elastic force vector as described in Section 17.

ANCF RI $\varepsilon_{m}$

The term in the elastic force vector (see Equation (17.50)) associated to the inplane strains (see Equation (17.51)) is evaluated using $2 \times 2$ Gaussian quadrature.

ANCF RI $\varepsilon_{m}+\gamma_{t}$

The term in the elastic force vector (see Equation (17.50)) associated to the inplane strains, $\varepsilon_{m}$, (see Equation (17.51)) and the transverse shear strains, $\gamma_{t}$, (see Equation(17.52)) are evaluated using $2 \times 2$ Gaussian quadrature.

ANCF RI $\varepsilon_{m}+$ ANS $\gamma_{t}$ The term in the elastic force vector (see Equation (17.50)) associated to the inplane strains, $\varepsilon_{m}$, (see Equation (17.51)) is evaluated using $2 \times 2$ Gaussian quadrature. The expression for the transverse shear strains, $\gamma_{t}$, is substituted with the expression for $\gamma^{A N S}$ given in Equation (18.56).

Table 3: Overview of notation used to describe the different combinations of locking remedies.

In order to obtain the numerical results, the element and the different locking remedies have been implemented in Matlab [52]. The static results are calculated using the iterative procedure described in Section 6.2. Here the $l^{2}$-norm of the residual vector is chosen as the convergence criteria and the equilibrium iterations are stopped when the norm is below $1 \times 10^{-4}$. When calculating the static equilibrium solutions, a value of $1 \times 10^{-8}$ is added to the perturbed coordinate when calculating the finite differences used to derive the tangent stiffness matrix. This value has been chosen empirically through numerical experiments where it has proven to give the fastest convergence 
19. Numerical examples

for the studied models. For other models and loading conditions, this value may not be optimal. The eigenfrequencies are obtained using the built-in Matlab function eig.

\subsection{Convergence study}

The quadratic element is subjected to a convergence study based on static analyzes of a cantilever plate with varying model parameters. A sketch of the cantilever plate with its applied load is shown in Fig. 29.

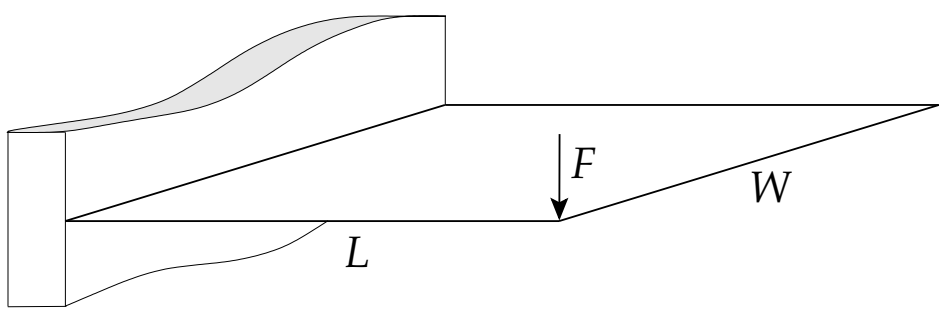

Fig. 29: Cantilever square plate with applied point load.

The plate is loaded in the transverse direction and the load, F, Young's modulus, E, and plate thickness, $h$, is varied. This is done in order to test the performance of the new element in both a small deflection, SD, and a large deflection, LD, problem and when applied in both thin and thick plate models. The plate is assumed to have a side length of $L=W=1 \mathrm{~m}$. The remaining parameters are load case specific and these are listed in Table 4.

For the convergence studies, results obtained using the quadratic ANCF shell element are compared to results obtained using the commercial static finite element solver Abaqus/Standard 6.14 [1] accounting for large deformations and using default solver settings. The cantilever plate is modeled using the eight noded S8R element which is a doubly curved thick shell element using reduced integration. This element has been chosen since it has several features in common with the developed quadratic shear and thickness deformable ANCF shell element. That being the eight noded topology, the capability of describing transverse shear strain and the use of reduced integration for alleviating locking.

The basis of the convergence study is the transverse deflection of 
the node where the point load is applied. Here, solutions are obtained for varying mesh sizes, ranging from $2 \times 2$ to $24 \times 24$ elements, and for each solution the absolute error with respect to a reference solution is calculated. The reference solutions are obtained using Abaqus models where the cantilever plate is modeled using $50 \times 50$, elements and the reference for each load case is shown in Table 4 . The convergence rates for the analyzed load cases are calculated as the absolute error with respect to their respective reference solution. For better comparison of the convergence rates, the absolute error is plotted versus the number of used elements on double logarithmic scales. Using this, the order of convergence can be read from the slope of the curves shown in the plots.

In the first convergence study, the effect of applying the reduced integration for the inplane strains, $\varepsilon_{m}$, is investigated. Here, the convergence rates of the ANCF RI $\varepsilon_{m}$ implementation is compared to the ANCF Unmodified and the Abaqus S8R element. The results are shown in Fig 30. In overall, both the ANCF Unmodified and the ANCF RI $\varepsilon_{m}$ has inferior performance when compared to the results obtained using Abaqus S8R elements. In particular for the thin plate problems (see Fig. 30a and Fig. 30c) where the Abaqus S8R elements has a second order convergence rate, the ANCF element gives only first order convergence.

In the case of the small deflection problems (Fig. 30a and Fig. $30 \mathrm{~b}$ ), the results appear unaffected by the use of RI on the inplane

\begin{tabular}{lrrrr}
\hline \multicolumn{5}{c}{ Load case } \\
\hline Property & Thin SD & Thick SD & Thin LD & Thick LD \\
\hline$E[\mathrm{~Pa}]$ & $2.1 \times 10^{11}$ & $2.1 \times 10^{11}$ & $2.1 \times 10^{11}$ & $2.1 \times 10^{8}$ \\
$v$ & 0.3 & 0.3 & 0.3 & 0.3 \\
$h[\mathrm{~m}]$ & 0.01 & 0.1 & 0.01 & 0.1 \\
$F[\mathrm{~N}]$ & 100 & 100.000 & 10.000 & 15.000 \\
\hline Reference $[\mathrm{m}]$ & $-2.5637 \times 10^{-3}$ & $-2.7154 \times 10^{-3}$ & $-2.2917 \times 10^{-1}$ & $-3.5155 \times 10^{-1}$ \\
\hline
\end{tabular}

Table 4: Parameters and reference solutions for the convergence study using the biquadratic shear and thickness deformable ANCF shell element. The reference solutions are obtained using Abaqus with a mesh size of $50 \times 50$ elements. 
19. Numerical examples

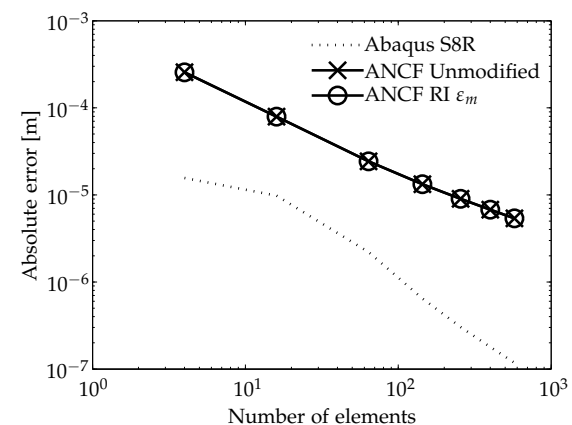

(a) Thin plate, small deflection.

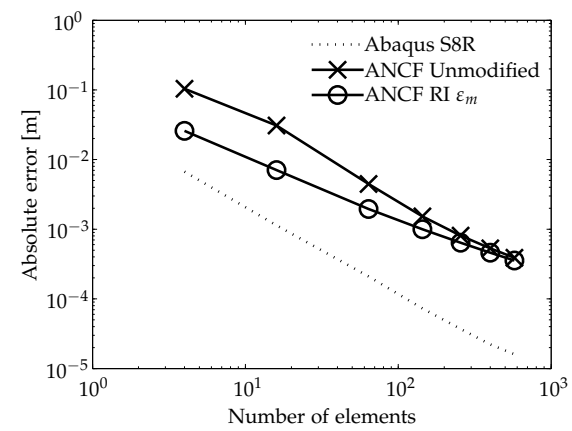

(c) Thin plate, large deflection.

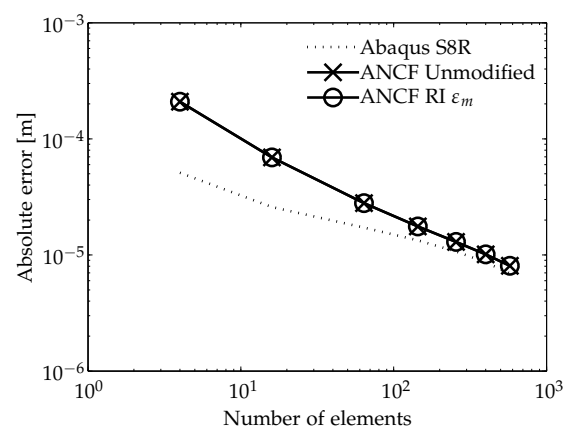

(b) Thick plate, small deflection.

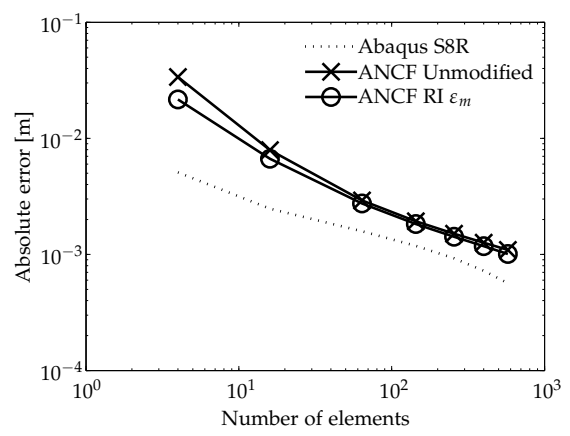

(d) Thick plate, large deflection.

Fig. 30: Convergence study on a square cantilever plate with a single point load in one corner using the quadratic ANCF shell element. The plots show the absolute error versus the number of elements when applying reduced integration to alleviate membrane locking. The absolute error is calculated with respect to a reference solution obtained using Abaqus.

strains. For the large deflection problems, however, the ANCF RI $\varepsilon_{m}$ gives slightly better results when a low number of elements is used. This is assumed to be due the fact that the RI on $\varepsilon_{m}$ alleviates membrane locking, which is present when the elements have noticeable curvature. As the element size reduces, the bending of each single element is reduced and the effect of membrane locking vanes.

This first convergence study has demonstrated that the quadratic ANCF shell element is suffering from severe locking problems. This is evident from the poor order of convergence which can be read from the convergence plots (see Fig. 30). Furthermore, the use of RI on $\varepsilon_{m}$ is necessary, as it improves the accuracy of the element when describing 


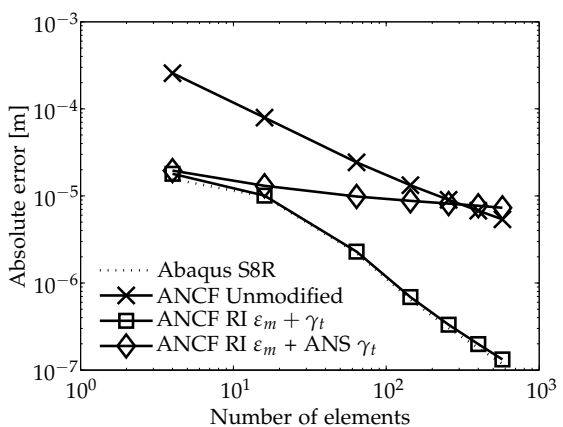

(a) Thin plate, small deflection.

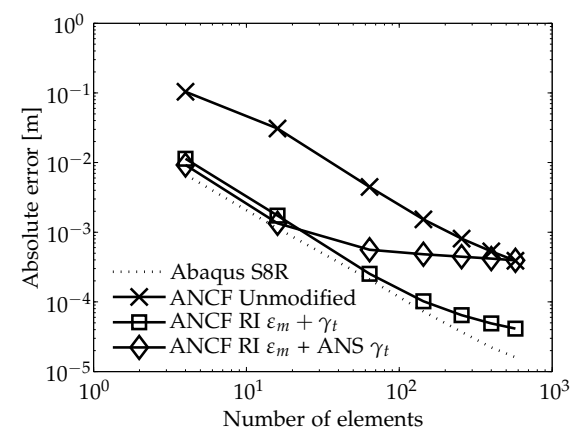

(c) Thin plate, large deflection.

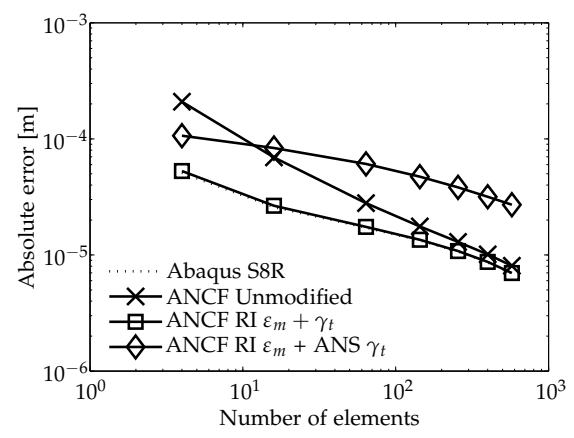

(b) Thick plate, small deflection.

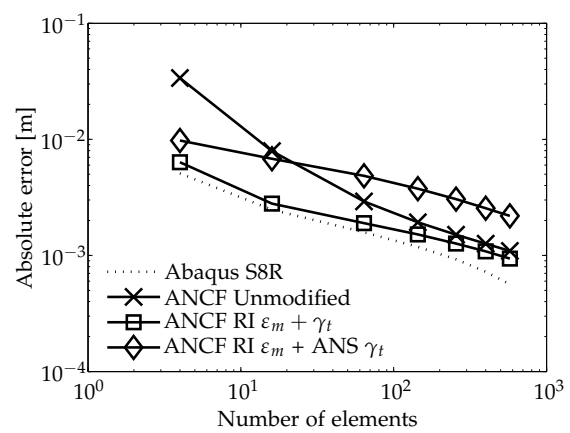

(d) Thick plate, large deflection.

Fig. 31: Convergence study on a square cantilever plate with a single point load in one corner using the quadratic ANCF shell element. The plots show the absolute error versus the number of elements when applying means to alleviate transverse shear locking. The absolute error is calculated with respect to a reference solution obtained using Abaqus.

problems with large curvature, but additional means are necessary to further enhance the performance of the element.

The next convergence study will present the results obtained using the ANCF RI $\varepsilon_{m}+\gamma_{t}$ and ANCF RI $\varepsilon_{m}+$ ANS $\gamma_{t}$ where means to address transverse shear locking have been introduced. The results obtained using these combinations are shown in Fig. 31 together with the results from the ANCF Unmodified and the Abaqus S8R.

When studying the convergence plots, two prominent observations can be made. First of all, the improved performance of the quadratic ANCF element when applying RI on the transverse shear strains, $\gamma_{t}$, is highly noticeable. Here it is seen that the ANCF RI $\varepsilon_{m}+\gamma_{t}$ gives 
identical results when compared to the Abaqus S8R for the small deflection problems (see Fig. 31a and Fig. 31b). For the large deflection problems (see Fig. 31c and Fig. 31d) the ANCF RI $\varepsilon_{m}+\gamma_{t}$ gives acceptable results, but with a slight deviation from those obtained using Abaqus S8R when a higher number of elements is used.

The second prominent observation is the performance of the ANCF RI $\varepsilon_{m}+$ ANS $\gamma_{t}$. This combination of locking remedies show a mediocre performance when compared to both the ANCF RI $\varepsilon_{m}+\gamma_{t}$ and Abaqus S8R, but also the ANCF Unmodified. Especially for the thin plate load cases (see Fig. 31a and Fig. 31c), where the ANCF RI $\varepsilon_{m}+$ ANS $\gamma_{t}$ converge to solutions that under-predict the magnitude of the deflection when compared to the Abaqus reference.

This second convergence study has compared the two investigated methods for treating transverse shear locking. The convergence study indicates that the use of RI is the most efficient method to treat the transverse shear locking. The ANCF RI $\varepsilon_{m}+\gamma_{t}$ has given results that are consistently more accurate than those obtained using ANCF RI $\varepsilon_{m}$ + ANS $\gamma_{t}$ for the four studied load cases. However, further analysis are needed to render the ANCF RI $\varepsilon_{m}+\gamma_{t}$ combination fully validated. For instance, the analyzed models have consisted of optimal elements of square shape with the midside nodes placed exactly on the element midsides. In the literature it is reported that the use of reduced integration in skew quadratic shell elements and elements where the midside node is not located in the middle can deteriorate simulation results [48]. Due to this, further studies on models of arbitrary shape and with distorted mesh is needed.

The small deviation between the ANCF RI $\varepsilon_{m}+\gamma_{t}$ and the Abaqus S8R noted in the large deflection load cases (see Fig. 31c and Fig. 31d) could indicate that the quadratic ANCF shell element still suffers from locking. This could prove to be thickness locking. During the convergence study, a combination of EAS and the ANCF RI $\varepsilon_{m}+\gamma_{t}$ was tested. Here, the simulations showed no change when compared to the results obtained using the pure ANCF RI $\varepsilon_{m}+\gamma_{t}$. Additionally, it was observed that the EAS parameters, $\alpha$, was equal to zero during all equilibrium iterations and when static equilibrium was obtained. This shows that the EAS did not become active. A locking mechanism that has not been treated yet is curvature thickness locking and an assessment of the presence this locking phenomena is given in Section 
19.3.

A last observation during the convergence study was the computational performance of the quadratic ANCF shell element. For all the load cases and mesh sizes, the load was applied in a single load step, which is possible due to the non-incremental form of the ANCF. When calculating the static equilibrium solutions for the small deflection load cases, between two and four equilibrium iterations was needed to obtain a converged results. In the large deflection load cases, the number of necessary iterations were between eight and twelve. Especially the later, is considered being a relatively low number in view of the magnitude of the resulting deflections and the very simple approach used to solve the equilibrium equations. For comparison, Abaqus took between eight and twelve load steps with up to three equilibrium iterations in each load step ${ }^{4}$. This testifies the robustness of the quadratic ANCF shell element and suggests that it could also be considered as a tool for general non-linear structural analysis.

\subsection{Eigenfrequency analysis}

When modeling structural dynamics using any finite element, it is important that the chosen element can describe the vibrating frequencies and associated mode shapes for the structure accurately. This is to ensure that the response due to time varying and/or oscillating loads is predicted correctly. The quadratic shear deformable element is tested in this discipline by applying it in a eigenfrequency analysis of a square plate with free boundary conditions on all edges. The model parameters are given in Table 5 .

In the convergence study it was shown that the ANCF RI $\varepsilon_{m}+\gamma_{t}$ appeared to be the most effective combination of the locking remedies that have been tested here. Due to this, only results using this combination will be shown and discussed. Additionally, results obtained using the ANCF Unmodified is listed in order to demonstrate the improvement when applying means to address locking. The obtained results are compared to analytical solutions based on the Rayleigh-Ritz method using polynomials as trial functions [69]. Furthermore, results from Abaqus will be used for comparison with a conventional finite

\footnotetext{
${ }^{4}$ These numbers are when using the default solver settings and can undoubtedly be lowered using of proper solver settings
} 
element solver. The results from Abaqus are obtained using the eight noded S8R element and the eigenfrequencies are calculated using the Lanczos solver with default settings [1].

The first ten non-zero eigenfrequencies calculated for the square plate are given in the Tables $6-8$, where they are listed for three different mesh sizes: $2 \times 2,4 \times 4$, and $8 \times 8$, respectively. The results show that the estimation of the eigenfrequencies is improved significantly when applying the reduced integration on the inplane and transverse shear strains. Especially for the higher order modes, the difference between the ANCF Unmodified and the ANCF RI $\varepsilon_{m}+\gamma_{t}$ is significant for all mesh sizes. However, as the mesh is refined the ANCF Unmodified is capable of predicting the eigenfrequencies to a satisfactory level.

When comparing the results obtained using the ANCF RI $\varepsilon_{m}+$ $\gamma_{t}$ to those obtained using Abaqus, it is highly noticeable how well they correlate. For every mode and mesh size, the results for the two elements are only deviating slightly in the decimal points. The only exception is the eighth mode calculated using a $2 \times 2$ mesh (see Table 6) where a slightly larger deviation is seen. In overall, the resemblance between ANCF RI $\varepsilon_{m}+\gamma_{t}$ and the Abaqus S8R is highly notable, and the small deviations might as well come from the different algorithms used to calculate the eigenfrequencies.

The mode shapes for the associated eigenfrequencies calculated using the ANCF RI $\varepsilon_{m}+\gamma_{t}$ have also been studied and compared qualitatively to those obtained using Abaqus with good agreement. However, these results are not shown here. In the future, the ability of the quadratic ANCF element to estimate correct mode shapes of vibrating plates should be documented properly. This could be done e.g.

\begin{tabular}{lr}
\hline Property & Value \\
\hline Side lengths & $1 \mathrm{~m}$ \\
Thickness & $0.01 \mathrm{~m}$ \\
Young's Modulus & $2.1 \times 10^{11} \mathrm{~Pa}$ \\
Poisson's ratio & 0.3 \\
\hline
\end{tabular}

Table 5: Parameters for the square plate subjected to an eigenfrequency analysis. 
Shear and thickness deformable ANCF shell elements

\begin{tabular}{crrrr}
\hline Mode & Reference [69] & ANCF Unmodified & ANCF RI $\varepsilon_{m}+\gamma_{t}$ & Abaqus S8R \\
\hline 1 & 35.0314 & 35.4369 & 35.4199 & 35.4194 \\
2 & 50.9709 & 58.3303 & 52.9955 & 52.9840 \\
3 & 63.1289 & 79.4337 & 67.1298 & 67.1225 \\
4 & 90.5204 & 105.6134 & 92.9102 & 92.8437 \\
5 & 90.5204 & 105.6134 & 92.9102 & 92.8437 \\
6 & 158.9068 & 187.1687 & 184.9904 & 184.9539 \\
7 & 158.9068 & 187.1687 & 184.9904 & 184.9539 \\
8 & 165.6533 & 197.1301 & 168.0171 & 167.4429 \\
9 & 180.1654 & 242.9386 & 195.5332 & 195.5005 \\
10 & 200.7284 & 579.1054 & 241.8092 & 241.7870 \\
\hline
\end{tabular}

Table 6: Ten first non-zero natural frequencies in $[\mathrm{Hz}]$ for a square plate with free boundary conditions obtained using a $2 \times 2$ mesh.

by drawing the so-called Chladni figures [96]. These figures show the nodal lines of the vibrating plates and has previously been used for validating other ANCF elements [69]. 
19. Numerical examples

\begin{tabular}{crrrr}
\hline Mode & Reference [69] & ANCF Unmodified & ANCF RI $\varepsilon_{m}+\gamma_{t}$ & Abaqus S8R \\
\hline 1 & 35.03139 & 35.3735 & 35.0590 & 35.0572 \\
2 & 50.97085 & 52.2516 & 51.1259 & 51.1249 \\
3 & 63.12889 & 65.6350 & 63.3909 & 63.3897 \\
4 & 90.52042 & 93.1212 & 90.1701 & 90.1541 \\
5 & 90.52042 & 93.1212 & 90.1701 & 90.1541 \\
6 & 158.9068 & 180.0979 & 163.0633 & 163.0512 \\
7 & 158.9068 & 180.0979 & 163.0633 & 163.0512 \\
8 & 165.6533 & 189.3308 & 170.3540 & 170.2097 \\
9 & 180.1654 & 195.3062 & 183.6735 & 183.6036 \\
10 & 200.7284 & 226.0742 & 207.2303 & 207.1484 \\
\hline
\end{tabular}

Table 7: Ten first non-zero natural frequencies in $[\mathrm{Hz}]$ for a square plate with free boundary conditions obtained using a $4 \times 4$ mesh.

\begin{tabular}{crrrr}
\hline Mode & Reference [69] & ANCF Unmodified & ANCF RI $\varepsilon_{m}+\gamma_{t}$ & Abaqus S8R \\
\hline 1 & 35.03139 & 35.0783 & 34.9128 & 34.9126 \\
2 & 50.97085 & 51.2525 & 50.9599 & 50.9597 \\
3 & 63.12889 & 63.6873 & 63.1134 & 63.1131 \\
4 & 90.52042 & 90.9773 & 90.1403 & 90.1383 \\
5 & 90.52042 & 90.9773 & 90.1403 & 90.1383 \\
6 & 158.9068 & 163.2497 & 159.0071 & 159.0054 \\
7 & 158.9068 & 163.2497 & 159.0071 & 159.0054 \\
8 & 165.6533 & 167.0145 & 164.7070 & 164.6933 \\
9 & 180.1654 & 183.2945 & 179.7501 & 179.7423 \\
10 & 200.7284 & 205.6709 & 200.6514 & 200.6404 \\
\hline
\end{tabular}

Table 8: Ten first non-zero natural frequencies in $[\mathrm{Hz}]$ for a square plate with free boundary conditions obtained using a $8 \times 8$ mesh. 


\subsection{Assessment of curvature thickness locking}

In the convergence study presented in Section 19.1, it was mentioned that the quadratic shear deformable ANCF shell element may suffer from curvature thickness locking. The presence of curvature locking in shear deformable ANCF elements has previously been discussed in the context of beam elements [26]. Here it was concluded that two noded shear deformable ANCF beam elements will suffer from curvature locking when they have non-parallel transverse slope vectors, as shown in Fig. 32. The same behavior is mentioned in the bilinear shear deformable ANCF shell element [98, 92].

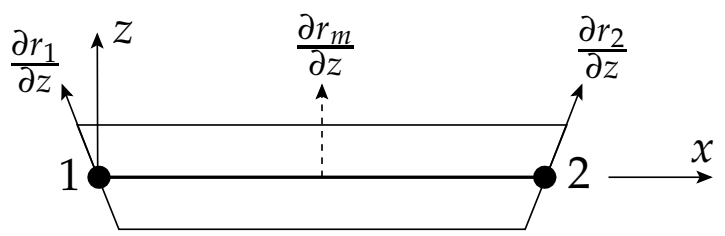

Fig. 32: Side view of two noded ANCF element with non-parallel transverse slope vectors.

The cause of curvature locking in linear shear deformable ANCF elements, is that the transverse slope vector is interpolated linearly. In the case where these elements have non-parallel transverse slope vectors, e.g. in the case where they exhibit pure bending (see Fig. 32), the $\mathrm{z}$-coordinate of the slope vector will be constant along the span of the beam element. The x-coordinate, however, will vary linearly from a negative value at node 1 to a positive value at node 2 . This will lead to change in length along the beam span of the slope vector, having a minimum value at the center represented by $\partial r_{m} / \partial z$ in Fig. 32. This corresponds to a strain state where the element is contracting in the thickness direction along the span of the element. This is an artificial strain state which is caused purely by the interpolation of the transverse slope vectors.

To alleviate this artificial thickness straining, it was proposed to introduce an extra node in the middle of the shear deformable beam element. Using this, the element is capable of describing curvature correctly and an significant improvement in accuracy was demonstrated [26]. 
The concept of introducing the midside node was adopted when formulating the eight noded quadratic shear deformable ANCF shell element which gives the ability to describe curved shapes. However, since the eight noded element topology was chosen, the element does only have midside nodes at the edges of the element meaning that a node in the center is lacking. This could mean that curved and especially doubly curved shapes cannot be described in an optimal manner. As a consequence, it is possible that the lacking node in the center of the element can cause curvature thickness locking when describing curved shapes.

To test this hypothesis, the transverse normal strain will be calculated for the quadratic ANCF shell element for out-of-plane curved configurations. The curved configurations are constructed by positioning a square quadratic ANCF shell element with side lengths equal to $1 \mathrm{~m}$ and a thickness of $0.2 \mathrm{~m}$ symmetrically around the origin of a coordinate system $O$ (see Fig. 33). Then the element is bent out of plane with a radius, $r$, chosen to be $1.5 \mathrm{~m}$. Using these parameters, the nodal coordinates and slope vectors for the out-of-plane curved element is calculated. The slope vectors are calculated as normalized vectors originating at point $Q$ pointing towards each node. In the first case, the element is singly curved with respect to the y-axis (see Fig.

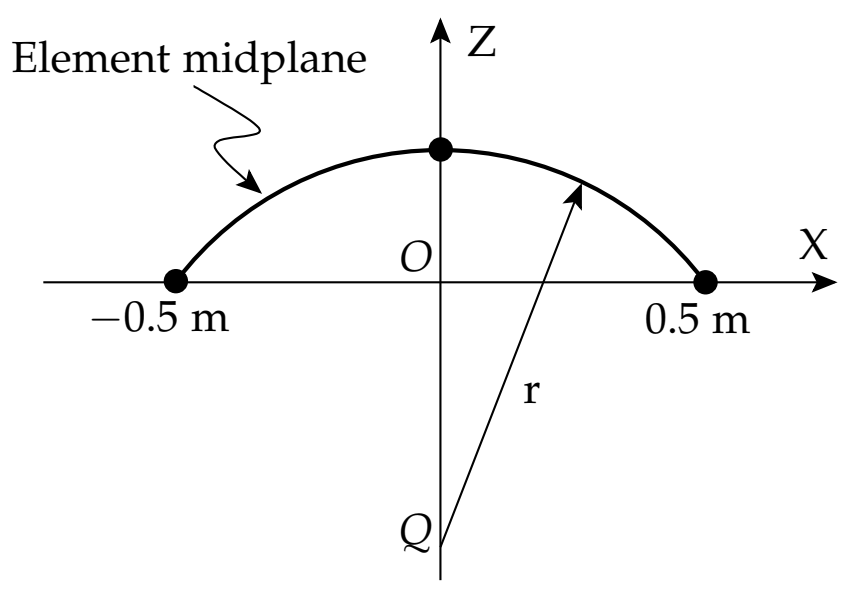

Fig. 33: Side view of a quadratic ANCF shell element in a singly curved configurations with radius $r$. The element is shown without the transverse slope vectors for brevity. However, these are unit vectors in the radial direction. 
34), and a set of nodal coordinates representing this shape is calculated directly using the mentioned parameters. In the second case, the element is curved along both the $\mathrm{x}$ and the $\mathrm{y}$-axis leading to a doubly curved shape (see Fig. 35). The coordinates for this configuration can be found by superimposing a set of nodal coordinates describing a curved element with respect to the $x$-axis on the configuration shown in Fig. 34 and re-calculating the slope vectors to fit the doubly curved shape. It should be noted that these configurations are not actual physical configurations, as aspects such as the anticlastic effect is neglected. They are entirely meant for demonstration purpose of the artificial thickness strain arising due to the curved configuration.

The transverse normal strains for the two curved configurations are calculated using Equation (17.53) and the results are shown in the contour plots in Fig. 36. In the figures, the colors represent the magnitude of the transverse normal strain with the darkest areas having the largest numerical value. By inspection of the contour plots, it is immediately seen that the thickness strain is non-zero in large areas of the elements and the magnitude varies over the elements.

When studying the strain state in the single curved element (see Fig. 36a), it is seen that the non-zero strains are located in two parallel bands which lie parallel with the x-axis. Along both of the element edges parallel to the $\mathrm{x}$-axis and in a band across the element center, where $y=0$, the thickness strain is zero. This tendency with the contraction between the nodes has been observed previously in Fig. 17b when the conventional Q8 element was used to discretize a a hollow circular plate.

In the case of the doubly curved element (see Fig. 35 and Fig. 36b), it is seen that the thickness strain is concentrated in the center of the element and fades towards the element edges. Again, it is noted that the thickness strain is zero at along the edges where the nodes are located.

When comparing the magnitudes of the thickness strains in the two studied cases, it is seen that the maximum strain is approximately 22 times larger in the doubly curved configuration than in the singly curved configuration. This indicates that the curvature locking in the quadratic ANCF shell element is most prominent when describing doubly curved shapes. Another interesting fact can be found when comparing the thickness strain to the magnitude of the bend- 
19. Numerical examples

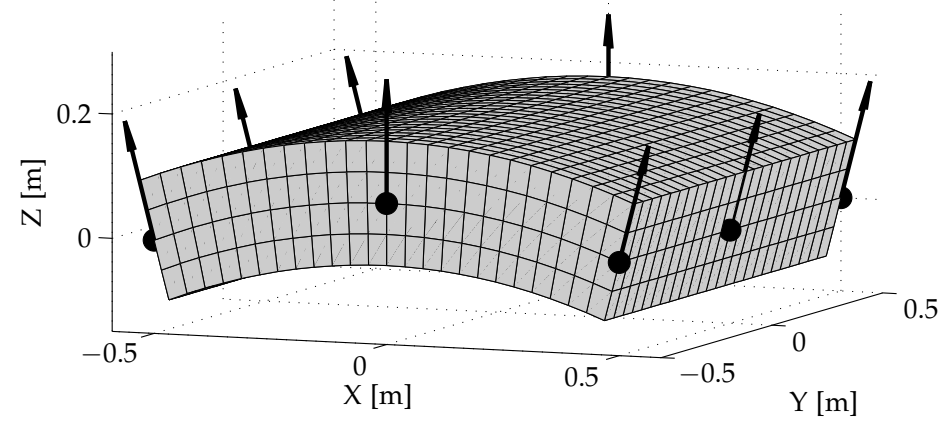

Fig. 34: Quadratic ANCF shell element in a curved configuration.

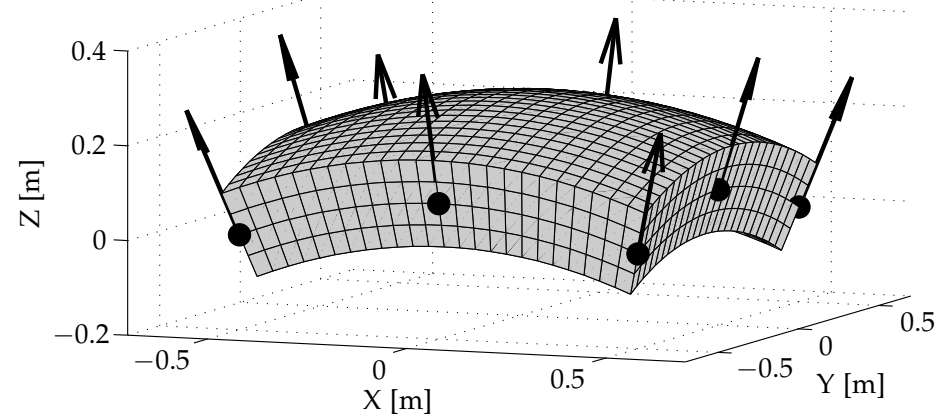

Fig. 35: Quadratic ANCF shell element in a doubly curved configuration.

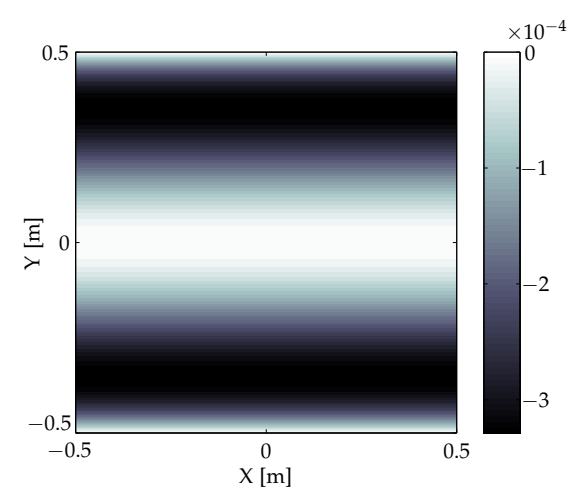

(a) $\varepsilon_{z z}$ in curved configuration.

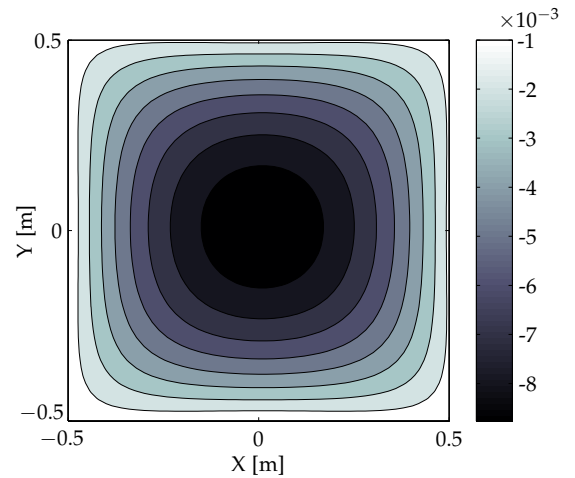

(b) $\varepsilon_{z z}$ in doubly curved configuration.

Fig. 36: Contour plots showing the transverse normal strain, $\varepsilon_{z z}$, arising in a quadratic shear and thickness deformable ANCF shell element. 


\begin{tabular}{crr} 
Maximum strain & Singly curved & Doubly curved \\
$\varepsilon_{x x}^{b}$ & $7.169 \times 10^{-2}$ & $6.723 \times 10^{-2}$ \\
$\varepsilon_{z z}$ & $4.083 \times 10^{-4}$ & $8.764 \times 10^{-3}$ \\
\hline
\end{tabular}

Table 9: Absoulute values of the maximum bending normal strain, $\varepsilon_{x x}^{b}$, and transverse normal strains, $\varepsilon_{z z}$, for the two studied curved configurations.

ing normal strains in the elements arising to the element curvature. The maximum bending strain is calculated by multiplying the curvature, as calculated in Equation (17.54), by half of the plate thickness, i.e. $\varepsilon_{x x}^{b}=\frac{h}{2} \kappa_{x x}$. The maximum bending strain in the x-direction, $\varepsilon_{x x}^{b}$, and the maximum transverse normal strain for both configurations are shown in Table 9. Here, it is seen that the maximum bending strain, for both configurations, is approximately $7 \times 10^{-2}$. By comparison of the strain magnitudes, it is seen that the thickness strain is 175 times smaller than the bending strain in the singly curved configuration, rendering it practically insignificant compared to the dominating bending strain. In the doubly curved configuration, however, the thickness strain is only approximately 8 times smaller than the bending strain. This means that the artificial thickness strains can have considerable magnitude compared to the bending strains in doubly curved configurations which could affect the performance of the element. Such behavior was noted previously in the large deflection load cases in the convergence study (see Section 19.1).

This small investigation on the presence of curvature thickness locking in the quadratic ANCF shell element has showed that this locking mechanism may arise, especially when describing doubly curved shapes. Due to this, a suitable method to alleviate this locking phenomena must be chosen. In previous studies, Lobatto quadrature has been applied for addressing the curvature locking in ANCF beam elements (e.g. [55]). Here, the term in the elastic force vector associated to thickness straining is evaluated using Lobatto quadrature instead of Gaussian quadrature. The argument of using this different quadrature rule, is that the limits of the integral is included in the sampling points. This means that the integrand will be evaluated at the nodal locations when the Lobatto quadrature is applied for finite elements. However, 
20. Discussion on shear deformable shell finite elements for non-linear analysis

other sampling points are located in between the nodal points, e.g. in the element center for a third order rule which is where the largest artificial thickness strain is located for the doubly curved configuration. Due to this, it is uncertain that Lobatto quadrature can be applied to alleviate curvature thickness locking in the proposed element.

A obvious choice for addressing curvature thickness locking would be an ANS method as it has been applied in the linear shell element $[98,92]$. Here, the transverse normal strain is sampled at the nodal locations and redistributed using bi-linear interpolation functions. A similar approach can be adopted in the case of the quadratic element and an indication of the effect can be obtained by inspection of the contour plots in Fig. 36. Here, it is seen that the artificial thickness strains are zero at the nodal locations, which is due to the fact the the slope vectors here are defined as unit vectors. It is assumed that the ANS approach could be a candidate of alleviating the curvature locking in the quadratic ANCF shell element. The specific approach could be a combination of sampling the transverse normal strains at the nodal locations and redistribution them using the same interpolation functions as used in the kinematic description (see Equation (16.48)). However, it must be left for future studies to investigate this hypothesis, as it has not been possible within this project.

\section{Discussion on shear deformable shell finite el- ements for non-linear analysis}

During the numerical studies (see Sections 19.1 and 19.2), a good correlation between the quadratic shear deformable ANCF shell element and the Abaqus S8R element was observed. This has raised a natural question on whether the shear deformable ANCF shell elements share any similarities with existing traditional shell finite elements. In this section, available shell finite elements for non-linear structural analysis will be briefly reviewed and similarities between those and the shear and thickness deformable ANCF shell element will be pointed out.

Shell finite elements for non-linear analysis of shell structures have been under development for nearly half a decade. These shell finite elements have a proven track record in the analysis of problems including large deformations and non-linear material behavior. This 
means that their kinematic and elastic formulations are considered well-tested and robust. The degenerated shell element by Ahmad, Irons, and Zienkiewicz [2], also commonly referred to as the AIZ-shell element, was a first attempt to formulate a curved shell element capable of describing shear deformation. In the AIZ element, an inextensible director vector is utilized to describe the orientation of the cross section. The orientation of the director vector is updated using rotation parameters along the element sides. The director vector remains straight at all times which enforces a commonly applied assumption, namely that the cross section remains straight during deformation. It does not, however, limit the cross section to remain perpendicular to the midplane by which the capability to describe transverse shear deformation arises. The director vector is chosen to be an inextensible unit vector since it solves some numerical issues associated to thickness deformation in the case of thin elements [2]. Other examples of shear deformable shell elements based on director vector kinematics are the MITC elements [22] and the geometrically exact shell element [80].

In order to enhance modeling in problems where thickness deformation may have a significant effect, such as problems including contact, surface loads, and composite shells, a geometrically exact shell element with an extensible director field has been introduced [82]. This, of course, reinstates the problems that led to the use of an inextensible director in the AIZ element. These problems are handled by decomposing the extensible director field into a unit vector that defines the orientation and a non-constant scalar value that defines the magnitude of the director vector. Another benefit that arises by introducing the extensible director field, is that the need of rotational parameters vanishes since the director vector field can be updated using simple vector additions to determine the new tip point position of the director vector. Furthermore, since the extensible director vector makes it possible to describe thickness deformation, it is easier to implement general constitutive relations for accurate modeling of non-linear material behavior. Shell elements with extensible director kinematics has been further improved in order to address locking problems [10, 12].

A significantly different element for analysis of shell structures, is the so-called solid-shell element [33]. Instead of discretizing the midplane of the structure and use a vector to define the cross section, 
the solid-shell element discretizes the top and bottom surfaces of the structure and then spans the cross section between these using linear interpolation. This leads to a kinematic description that does not rely on rotational parameters, since it is based entirely on the positions of the nodes on the top and bottom surfaces. Furthermore, this kinematic description leads to an element that can describe thickness deformation since the relative distance between the nodes on the top and bottom surfaces is not fixed. Moreover, the two nodes in the thickness direction, makes capable to act as a transition element between solid finite elements and shell elements. Like the elements based on the director vector kinematics, the solid-shell element suffers from locking mechanisms which have been treated using various combinations of ANS and EAS [32, 95, 94].

The shear and thickness deformable ANCF shell elements share many characteristics with the director based shell elements and the solid-shell element that have been briefly reviewed here. The ANCF elements are clearly closest to the degenerated and geometrically exact shell formulations since these element families uses vector kinematics to describe the cross section orientation and deformation. The solidshell elements uses only nodal positions in the kinematic description, but the linear interpolation in the thickness direction makes it similar to the vector based kinematics. Many of the same techniques, such as ANS, EAS and reduced integration, used in the director based and solid-shell elements to address locking problems have been applied in the shear deformable ANCF shell elements. That being for both the bilinear element and the proposed quadratic element. In overall, the similar kinematic description and the use of well tested methods for alleviating locking should give the shear and thickness deformable ANCF shell elements similar performance compared to the director based and solid-shell elements. However, a thorough study based on both analytical discussions and numerical experiments must be carried out, though, to substantiate this hypothesis.

\section{Interim conclusion}

This third part of the thesis have concerned the development of a new quadratic ANCF shell element. Initially, the shortcomings of existing 
ANCF shell elements was briefly highlighted. This was done in order to justify the development of yet another ANCF element to be added to the increasing number of elements in the ANCF library. It was concluded that a versatile element family that performs well in both thin and thick plate problems was lacking, which had led to the development of the bilinear shear and thickness deformable element. This element, however, is not optimal for describing curved structures which explains the need for a quadratic shear and thickness deformable element.

The choice of parameterization and, especially, the chosen number of nodes for the quadratic element was discussed thoroughly. First of all, the omission of the inplane slopes was discussed. The absence of midplane slopes is fundamentally different than both the fully parameterized and the thin gradient deficient elements discussed earlier where slope vectors associated to element midplane are used. However, it was concluded that improved modeling of curved structures could be obtained by using additional midside nodes instead of inplane slope vectors. It was chosen to base the quadratic ANCF shell element on the eight noded serendipity topology due to the prospect of simple and straightforward methods for addressing problems regarding locking. After deciding on the element topology, the kinematics of the proposed element was discussed in detail. Here the vector of nodal coordinates and associated interpolation shape functions was introduced and the element's ability to describe transverse shear and thickness was highlighted.

The vector of elastic forces for the proposed element was derived in detail based on an elastic midplane approach. It was emphasized that the calculation of the element curvature takes a different approach than the one used for the thin plate element. The approach chosen for the quadratic ANCF element relies purely on first order derivatives of the position field, and an assumption based on the transverse slope vector being approximately normal to the element midplane. The use of only first order derivatives in the calculation of the curvature was mentioned to improve the accuracy of the curvature. This hypothesis and the validity on the assumption regarding the transverse slope vector must be investigated in future studies.

Three different methods for alleviating locking in the quadratic ANCF shell element was reviewed. Here, focus was on methods that 
have a proven track record in similar quadratic shell elements. The benefits and drawbacks for each method were discussed and their specific procedure for addressing locking problems in the proposed element was reviewed.

The performance of the proposed element was assessed using numerical examples. The first was a convergence study on the deflection of a cantilever plate. Here, the element was tested in both small and large deflection problems and for both thin and thick plate structures. The convergence study showed that the quadratic element suffers from extensive locking problems that impair its performance. However, the quadratic element showed good performance when appropriate means for addressing these locking problems were applied. Here, especially the reduced integration gave significant improvements. Furthermore, the convergence study indicated that the proposed ANCF shell element could be a valuable tool in general static non-linear problems, as it showed good performance in terms of the number of iterations used to obtain static equilibrium. Eigenfrequency analyses were conducted in order the test whether the proposed element can estimate the natural vibration frequencies of shell structures. Here, the results obtained using the quadratic ANCF shell element was compared with analytical results with good correlation.

A slight deviation in the convergence behavior was found for the large deflection load cases, and this raised a question on whether the quadratic ANCF shell element suffers from curvature thickness locking. The presence of curvature thickness locking was analyzed by studying the transverse normal strain in both singly and doubly curved elements. Here, it was noticed that significant thickness straining occurred when the element was placed in a doubly curved configuration which demonstrated that the element will suffer from curvature thickness locking. A possible method to alleviate this locking phenomena is briefly mentioned but has not been tested.

During the convergence and eigenfrequency studies, a noticeable resemblance between the ANCF RI $\varepsilon_{m}+\gamma_{t}$ and the Abaqus S8R was observed. This led to a review on similar shell elements for non-linear structural analysis and similarities between the reviewed elements and the shear and thickness deformable ANCF shell element were pointed out. The similarities lie in the vector based kinematic description and the applied means to alleviate locking. It was concluded that the sim- 
ilarities with the already proven and validated traditional shell finite element should give the shear and thickness deformable ANCF shell elements similar performance, but further studies are needed to confirm this.

In overall, this study have introduced a new quadratic shear and thickness deformable ANCF shell element. During the development, it has been chosen to glance at the formulation and applied techniques in other successful non-linear shell finite elements. This is an intentional choice in order not to reinvent the wheel, so to speak. However, the results and conclusions given here are in no terms final, as the development is still in an early state and further verification is needed. The proposed element is still to be tested in configurations with nonrectangular elements and transient dynamic studies must be carried out. The element should also be benchmarked against other methods such as analytical solutions or preferably accurate physical tests. Additionally, the benchmark test should not only be limited to comparison of displacements, but also include calculation of stresses, forces and moments for a wider picture of the performance.

Another aspect that may need to be reconsidered, is the choice of the eight noded topology used in the formulation of the quadratic element. Early in the process the eight noded topology was chosen over the nine noded, due to a simpler and more straightforward implementation. However, as the development of the eight noded element progressed, several places in the literature were noted where the nine noded topology is recommended over the eight noded. That being especially for cases where elements will have curved sides. Of course, in order to investigate which topology is best suited as base for a quadratic ANCF shell element, a nine noded variant must be implemented for comparison with the eight noded version.

One specific area where the nine noded topology is expected to improve the performance is when modeling doubly curved structures. This is due to the introduction of the node in the element center and the additional polynomial term in the kinematic description. The introduction of a transverse slope vector at the element center could also prove to be beneficial in terms of locking where it is assumed to reduce the artificial thickness straining when describing doubly curved structures.

Whether or not the eight noded shear and thickness deformable 
21. Interim conclusion

element proves to be preferred choice, much valuable and informative experience have been gained in the context of developing a quadratic ANCF shell element. This experience will be used in future investigations such that, in time, a robust and versatile quadratic ANCF shell element is developed. 
Shear and thickness deformable ANCF shell elements 


\section{Closing}





\section{Closing}

\section{Discussion and conclusion}

This PhD project has covered the modeling of flexible structural components that undergo large deformations and rotations. Here, focus has been on the analysis of shell structures using rectangular shell finite elements based on the absolute nodal coordinate formulation, ANCF. In order to initiate the study and obtain a general knowledge on the topic, available rectangular ANCF shell elements were reviewed in an early stage. During this process, a new shell element based on the combined arbitrary Lagrange-Euler, ALE, and ANCF methods was developed. It is believed that the ALE-ANCF formulation could prove useful e.g. in terms of enhanced modeling of sliding forces and joints, as a mesh refinement technique and for analysis of crack propagation.

The review of the existing ANCF shell elements and, in particular, the development of the ALE-ANCF shell element shed light over some possible issues regarding three thin ANCF shell elements. A thorough discussion of their kinematic descriptions highlighted differences regarding the inter-element continuity, namely whether $C^{0}$ or $C^{1}$ continuous displacement fields were ensured. Subsequently, complications regarding sensitivities to abrupt changes in element sizes, discontinuous representation of curved structures, and non-monotonic convergence when analyzing curved structures were addressed. These studies concluded that thorough convergence analyses and comparison with reference models are needed when applying the thin ANCF shell elements in real life design tasks. As a remedy to the poor performance of the thin ANCF shell elements, a modified kinematic description was suggested. However, this turned out to be unsuccessful as it did not improve their performance. 
In the last part of this $\mathrm{PhD}$ thesis, a new eight noded quadratic ANCF shell element was introduced. The kinematics of this element was based on an approach giving it capabilities of describing both transverse shear and thickness deformation along with the usual membrane and bending deformations. The calculation of the internal elastic forces was based on an elastic midplane approach and possible remedies to alleviate locking mechanisms were reviewed. Those being methods that have been developed for use in similar traditional finite elements. In order to test the performance of the proposed element, numerical studies were conducted. Apparently, the results look promising but additional work is needed to fully validate the element and to treat problems regarding curvature locking. Conclusively it is noted that the eight noded topology may not be the optimal choice for the quadratic ANCF shell element, but further studies are needed to investigate this.

In addition to the theoretical work presented here in this thesis and in the published papers (see [38, 40,39, 41]), the author have been involved in two studies where the ANCF is applied in real life engineering problems (see $[59,37]^{5}$ ). Despite not being included directly in the thesis, these studies have helped to keep the practical application of the ANCF in mind.

The project started out with the intention of extending the traditional flexible multibody dynamics approach, based on the floating frame of reference formulation, to be able to describe large deformations. In its early stage, however, the project took a turn towards understanding and development of ANCF based finite elements with the focus on multibody dynamics kept intact.

In all, this study has discussed available rectangular ANCF shell elements and added two new shell elements to the ANCF library. Hopefully, other researchers and engineering professionals can find use in reading the published papers and this thesis to gain further insight in rectangular ANCF shell elements. It is believed, that in time, the ANCF can prove to be a valuable engineering design tool that can supplement already existing methods for modeling flexible components in the multibody dynamics framework.

\footnotetext{
${ }^{5}$ These studies were conducted by students enrolled at the master's programme in mechanical engineering at Aarhus University. Here the author of this thesis was involved as a co-supervisor along with the supervisor of this PhD project.
} 


\section{Perspectives}

Even though this project has covered many aspects concerning the ANCF, it leaves a notion that it has raised more questions than it has answered. That being both questions and hypotheses written explicitly, but also more general thoughts and perspectives regarding the ANCF that have not been mentioned in the main body of the thesis. This last section aims to collect some previously mentioned aspects that must be explored, but also make suggestions, in a wider sense, for possible research areas within the ANCF.

A recurring question, throughout the entire thesis, concerns the calculation of the element curvature. This is a very important quantity when the internal elastic forces are derived using a structural mechanics approach. It could be interesting to compare results from identical element formulations where different ways of calculating the element curvature is used. Such an investigation could aim to give a mathematical discussion of different applied methods and include numerical examples where the methods are compared. The outcome of such a study could be a recommendation on which approach should be preferred over others for specific elements and applications.

The conclusive discussion regarding the ALE-ANCF shell element (see Section 7.4) mentions several aspects that calls for attention and further investigations. In general, what has been shown in this thesis is merely a proof of concept. A more sophisticated implementation is needed, such that the mesh is updated when a moving node approaches another node. If such an algorithm is not present, it will obviously lead to numerical problems in the limit where element sizes are approaching zero. Additionally, it must be tested whether it is necessary for an entire mesh to be made of ALE-ANCF elements or if they can be placed in a special area of interest only. This could drastically reduce the modeling complexity. An attempt should be made to implement the ALE-ANCF approach in a different element formulation, e.g. the shear and thickness deformable ANCF shell element. This is necessary in order to make the method applicable in situations where the thin plate assumption is invalid.

A variety of possible applications of the ALE-ANCF method is also mentioned in Section 7.4. That being applications such as modeling of moving boundary conditions, mesh refinement technique, and simu- 
lation of crack propagation. Especially the latter is a very interesting application. Using the ALE-ANCF, a crack could be modeled by placing a moving node at the crack tip to simulate the propagation of the crack through the modeled specimen. It is expected that a combination of describing plasticity in flexible components and crack propagation using the ALE-ANCF method could have huge impact in the future use of multibody dynamics.

In Part II, the performance and behavior of thin ANCF shell elements was discussed. This discussion highlighted several issues and problems regarding this element, and, in general, made this element type appear erroneous. It could be interesting to apply the element in a real life design problem, in order to find out whether these highlighted issues have any practical consequences.

The quadratic shear and thickness deformable ANCF shell element, which was introduced in Part III, is probably one of the subjects considered in this thesis, that still needs the most work. The basic formulation appears to be sound, but the element needs to be generalized into a form where initially non-rectangular and curved elements can be described. This involves that the initial shape of the element is taken into account when formulating the vector of elastic forces. The calculation of the elastic forces should also be expanded to include a description based on a continuum mechanics approach. Only then, the element would be able to describe arbitrary materials using non-linear constitutive relations. Additional means to alleviate locking are also necessary to ensure that the element is locking-free when using both the structural mechanics and the continuum mechanics approach. It should also be considered to explore the possibility using a nine noded element topology for the quadratic ANCF shell element instead of the eight noded topology which has been used in this study. This should be done with the intension of ensuring the most accurate, efficient and robust quadratic ANCF shell element as possible.

In terms of validation and benchmarking of the quadratic ANCF shell element, the test series should be expanded beyond static analyses of simple square plates. Here it is especially important that the numerical studies include analyses of complex structures in order to ensure that the element does not fail when applied in real life engineering problems. This concerns especially that the chosen locking remedies should maintain their effectiveness when elements have 
a non-optimal shape. Additionally, it could be interesting to compare the shear and thickness deformable ANCF shell element to the thin ANCF shell elements. This should be done to investigate if the shear and thickness deformable element can match the thin element in terms of computational performance when the shell thickness becomes small.

Seen in wider perspective, it is believed that the ANCF provides new and interesting opportunities for the modeling of problems that have earlier been very cumbersome or, in some cases, impossible. Previously, when modeling flexible components that undergo large deflections, the most practical and commonly used approach was to use sub-structuring techniques. Here the component of interest is segregated into several linear elastic flexible bodies that are fixed together using constraints. Now, by the use of ANCF, such components can be modeled directly. This reduces the modeling effort as normal mesh refinement can be carried out in straightforward manner in the ANCF, whereas updating and determining the number of necessary sub-structures can be a time consuming and tedious task. This will make it easier to model parts that have a nonlinear nature such as vehicle suspension components, wind turbine blades or leaf springs.

With the prospect of being able to incorporate nonlinear material models, it will be possible to enhance the modeling of machine parts made of e.g. rubber or composite materials. This would lead to increased modeling fidelity of e.g. belt drive systems, tires or, again, wind turbine blades. It could also be feasible to include plastic deformations and yielding of machine components. This could make it possible to simulate a critical overload of crucial machine components, leading to permanent deformations, and then capture its effect on the overall system performance in the moments after the overload. In the extreme case, it would be possible simulate complete failure of components, e.g. in combination with the aforementioned crack propagation technique. Using such a technique, design engineers could gain valuable insight in possible failure modes and their impact on the remaining system.

The use of ANCF will bring nonlinear finite element modeling and multibody dynamics closer together, which can prove to take virtual prototyping to the next level. The nonlinear finite element modeling will benefit from always having accurate loads from the overall system 
that they are part of. The multibody dynamics model, on the other hand, will benefit from being able to capture the response of nonlinear flexible components that will affect the overall performance of the modeled system. This combination will lead to a more integrated and versatile analysis tool.

Despite the high expectations and the improved opportunities to reduce the use of physical prototypes, the promises stated here should be taken with caution. The ANCF still needs a lot of research and development before it can be realized in the terms described here. Furthermore, it should be adopted in commercially available software packages, such that it can be used as a daily engineering tool. The ANCF is well suited for problems such as rubber belts, cables, and flexible wings. However, for other applications where components undergo limited or no deformations, more efficient modeling is obtained by using already existing methods such as the floating frame of reference formulation. Hence, the ANCF should not be seen as 'the wonder of analysis tools' but as a promising supplement to the already existing suite of engineering tools. The ANCF is still young, but it is believed that it can prove to be useful in the development of the products of tomorrow.

\section{Afterword}

Seen in a wider perspective, it has been a very educational experience doing research in the intersection between multibody dynamics and the finite element method. Personally, this has given much valuable experience and insight that will prove to be beneficial in a future career, whether that being in the industry or in academia. It has been liberating to be allowed and able to take the project in the direction which felt right, and not being limited by a fixed project description. If not possible, this would undoubtedly have made the project more burdensome. It has been exciting to be at the forefront of the development of the ANCF and contribute to this highly active research field and it will be, if possible, even more exciting to follow and participate in it, in the future. 


\section{References}





\section{References}

[1] Abaqus/CAE. version 6.14 User's Guide. Dassault Systèmes Simulia Corp., Providence, RI, USA, 2014.

[2] Ahmad, S., Irons, B. M., and Zienkiewicz, O. C. Analysis of thick and thin shell structures by curved finite elements. International Journal for Numerical Methods in Engineering 2 (1970), 419-451.

[3] Andelfinger, U., AND Ramm, E. Eas-elements for twodimensional, three-dimensional, plate and shell structures and their equivalence to hr-elements. International Journal for Numerical Methods in Engineering 36 (1993), 1311-1337.

[4] BAK, M. K., ANd Hansen, M. R. Analysis of offshore knuckle boom crane - part one: Modeling and parameter identification. Modeling, Identification and Control 34(4) (2013), 157-174.

[5] BARLow, J. Optimal stress locations in finite element models. International Journal for Numerical Methods in Engineering 10 (1976), 243-251.

[6] Bauchau, O. A., Han, S., Mikkola, A., and Matikainen, M. K. Comparison of the absolute nodal coordinate and geometrically exact formulations for beams. Multibody System Dynamics 32 (2014), 67-85.

[7] Bayoumy, A. H., Nada, A. A., And Megahed, S. M. Modeling slope discontinuity of large size wind-turbine blade using absolute nodal coordinate formulation. Proceedings of the ASME 2012 IDETC \& CIE (2012).

[8] Bayoumy, A. H., Nada, A. A., and Megahed, S. M. A continuum based three-dimensional modeling of wind turbine blades. Journal of Computational and Nonlinear Dynamics (2013).

[9] Belytschko, T., Stolarski, H., Liv, W. K., Carpenter, N., AND ONG, J. S.-J. Stress projection for membrane and shear locking in shell finite elements. Computer Methods in Applied Mechanics and Engineering 51 (1985), 221-258. 
[10] Betsch, P., AND Stein, E. A nonlinear extensible 4-node shell element based on continuum theory and assumed strain interpolation. Journal of Nonlinear Science 6 (1996), 169-199.

[11] Blundell, M., ANd Harty, D. Multibody Systems Approach to Vehicle Dynamics. Elsevier Butterworth-Heinemann, Oxford, 2004.

[12] Büchter, N., Ramm, E., And Roehl, D. Three-dimensional extension of non-linear shell formulation based on the enhanced assumed strain concept. International Journal for Numerical Methods in Engineering 37 (1994), 2551-2568.

[13] Canavin, J. R., and Likins, P. W. Floating reference frames for flexible spacecraft. Journal of Spacecraft and Rockets 14(12) (1977), 724-732.

[14] Cook, R. D., Malkus, D. S., Plesha, M. E., and Witt, R. J. Concepts and applications of finite element analysis, $4^{\text {th }}$ ed. John Wiley \& Sons, Hoboken, 2002.

[15] Craig, R. R., and Bampton, M. C. C. Coupling of substructures for dynamic analysis. AIAA Journal 6(7) (1968), 1313-1319.

[16] Ding, J., Wallin, M., Wei, C., Recuero, A. M., and Shabana, A. A. Use of independent rotation field in the large displacement analysis of beams. Nonlinear Dynamics 76 (2014), 1829-1843.

[17] Dmitrochenko, O., Matikainen, M. K., and Mikкola, A. M. The simplest 3- and 4-noded fully parameterized ancf plate elements. Proceedings of the ASME 2012 International Design Engineering Technical Conferences \& Computers and Information in Engineering Conference (2012). Chicago, IL, USA.

[18] Dmitrochenko, O., and Mikкola, A. Two simple triangular plate elements based on the absolute nodal coordinate formulation. Journal of Computational and Nonlinear Dynamics (2008). DOI 10.1115/1.2960479.

[19] Dmitrochenko, O., And Pogorelov, D. Generalization of plate finite elements for absolute nodal coordinate formulation. Multibody System Dynamics 10 (2003), 17-43. 
References

[20] Donea, J., Huerta, A., Ponthot, J.-P., and Rodríguez-Ferran, A. Arbitrary Lagrangian-Eulerian methods, vol. 1: Fundamentals of Encyclopedia of Computational Mechanics. John Wiley \& Sons, Ltd., 2004, ch. 14.

[21] Dufva, K., And Shabana, A. A. Analysis of thin plate structures using the absolute nodal coordinate formulation. Journal of Multi-Body Dynamics 219 (2005), 345-355.

[22] Dvorkin, E. N., AND BATHE, K.-J. A formulatin of general shell element - the use of mixed interpolation of tensorial components. International Journal for Numerical Methods in Engineering 22 (1986), 697-722.

[23] Farouki, R. T., ANd Sakkalis, T. Real rational curves are not 'unit speed'. Computer Aided Geometric Design 8 (1991), 151-157.

[24] Farouki, R. T., and SaKkalis, T. Rational space curves are not 'unit speed'. Computer Aided Geometric Design 24 (2007), 238-240.

[25] Fujita, H., and Sugiyama, H. Development of flexible telescopic boom model using ancf sliding constraints with lugre friction. Theoretical \& Applied Mechanics letters 2 (2012).

[26] García-Vallejo, D., Mikkola, A., and Escalona, J. L. A new locking-free shear deformable finite element based on absolute nodal coordinates. Nonlinear Dynamics 50 (2007), 249-264.

[27] Gerstmayr, J., and IRschiK, H. On the correct representation of bending and axial deformation in the absolute nodal coordinate formulation with an elastic line approach. Journal of Sound and Vibration 318 (2008), 461-487.

[28] Gerstmayr, J., and Shabana, A. A. Analysis of thin beams and cables using the absolute nodal co-ordinate formulation. Nonlinear Dynamics 45 (2005), 109-130.

[29] Gerstmayr, J., Sugiyama, H., and Mikкola, A. Review on the absolute nodal coordinate formulation for large deformation analysis of multibody systems. Journal of Computational and Nonlinear Dynamics 8 (2012). DOI 10.1115/1.4025282. 
[30] Haastrup, M., Hansen, M. R., Ebbesen, M. K., and MouritSEN, O. . Modeling and parameter identification of deflections in planetary stage of wind turbine gearbox. Modeling, Identification and Control 33(1) (2012), 1-11.

[31] Haug, E. J. Computer Aided Kinematics and Dynamics of Mechanical Systems. Volume 1: Basic Methods. Allyn and Bacon, Boston, 1989.

[32] Hauptman, R., Doll, S., Harnau, M., and Schweizerhof, K. 'solid-shell' elements with linear and quadratic shape functions at larger deformations with nearly incompressible materials. Computers and Structures 79 (2001), 1671-1685.

[33] Hauptmann, R., and Schweizerhof, K. A systematic development of 'solid-shell' element formulations for linear and nonlinear analyses employing only displacement degrees of freedom. International Journal for Numerical Methods in Engineering 42 (1998), 49-69.

[34] Hong, D., ANd Ren, G. A modeling of sliding joint on onedimensional flexible medium. Multibody System Dynamics 26 (2011), 91-106.

[35] Hong, D., Tant, J., ANd Ren, G. Dynamic modeling of massflowing linear medium with large amplitude displacement and rotation. Journal of Fluids and Structures 27 (2011), 1137-1148.

[36] Hyldahl, P. Large displacement analysis of shell structures using the absolute nodal coordinate formulation. Technical Report ME-TR-6, Department of Engineering, Aarhus University, July 2013.

[37] Hyldahl, P., Andersen, S., Mikkelsen, S., and Balling, O. Modeling and feasibility study of nonlinear suspension components in multibody systems using absolute nodal coordinate formulation based beam elements -application to stabilizer bar. SAE International Journal of Passenger Cars - Mechanical Systems 8(2) (2015).

[38] Hyldahl, P., Mikкola, A. M., and Balling, O. A thin plate element based on the combined arbitrary lagrange-euler and absolute nodal coordinate formulations. Proceedings of the Institution 
References

of Mechanical Engineers, Part K: Journal of Multi-body Dynamics 227 (2013), 211-219.

[39] Hyldahl, P., Mikкola, A. M., and Balling, O. Studies on the kinematics of thin shell elements based on the absolute nodal coordinate formulation. Proceedings of the ASME 2005 International Design Engineering Technical Conferences \& Computers and Information in Engineering Conference (2014). Buffalo, New York, USA.

[40] Hyldahl, P., Mikkola, A. M., Balling, O., and Sopanen, J. T. Behavior of thin rectangular ancf shell elements in various mesh configurations. Nonlinear Dynamics 78 (2014), 1277-1291.

[41] Hyldahl, P., Mikkola, A. M., Balling, O., and Sopanen, J. T. Convergence characteristics of thin ancf shell elements in arbitrary and initially curved mesh. Proceedings of The 3rd Joint International Conference on Multibody System Dynamics \& The 7th Asian Conference on Multibody Dynamics (2014). BEXCO, Busan, Korea.

[42] KerkKänen, K. S., García-Vallejo, D., and Mikkola, A. M. Modeling of belt-drives using a large deformation finite element formulation. Nonlinear Dynamics 43 (2006), 239-256.

[43] Krenk, S. Non-linear Modeling and Analysis of Solids and Structures. Cambridge University Press, New York City, NY, 2009.

[44] KunL, D., And Ramm, E. Time integration in the context of energy control and locking free finite elements. Archives of Computational Methods in Engineering 7 (2000), 299-332.

[45] Larsen, T. J., and Hansen, A. M. How 2 HAWC2, the user's manual. Risø-R-1597(EN), 2007.

[46] LeE, J.-H., AND PARK, T.-W. Development of a three-dimensional catenary model using cable elements based on absolute nodal coordinate formulation. Journal of Mechanical Science and Technology 26 (2013), 3933-3941.

[47] LI, H., Yang, Z., AND HuAnG, T. Dynamics and elasto-dynamics optimization of a 2-dof planar parallel pick-and-place robot with flexible links. Structural and Multidisciplinary Optimization 38 (2009), 195-204. 
[48] MacNeal, R. H. Finite Elements: Their Design and Performance. Marcel Dekker, New York, 1994.

[49] Macneal, R. H., and Harder, R. L. Eight nodes or nine? International Journal for Numerical Methods in Engineering 33 (1992), 1049-1058.

[50] Matikainen, M., ANd Mikкоla, A. Improved description of elastic forces for the absolute nodal coordinate based plate element. In Proceedings of the ASME 2005 International Design Engineering Technical Conferences $\mathcal{E}$ Computers and Information in Engineering Conference (Long Beach, California USA, September 2005), ASME, pp. 1277-1283.

[51] Matikainen, M. K., Valkeapä̈̈, A. I., Mikkola, A. M., and SchwAB, A. L. A study on moderately thick quadrilateral plate elements based on the absolute nodal coordinate formulation. Multibody System Dynamics 31 (2014), 309-338.

[52] MATLAB. version 8.10 .604 (R2013a) Documentation. The MathWorks Inc., Natick, Massachusetts, 2013.

[53] Mikкola, A., and Shabana, A. A. A non-incremental finite element procedure for the analysis of large deformation of plates and shells in mechanical system application. Multibody System Dynamics 9 (2003), 283-309.

[54] Mohamed, A.-N. A. Three-dimensional fully parameterized triangular plate element based on the absolute nodal coordinate formulation. Journal of Computation and Nonlinear Dynamics 8 (2013). DOI 10.1115/1.4024729.

[55] Nachbagauer, K., Pechstein, A. S., Irschik, H., and GerstMAYR, J. A new locking-free formulation for planar, shear deformable, linear and quadratic beam finite elements based on the absolute nodal coordinate formulation. Multibody System Dynamics 26 (2011), 245-263.

[56] NADA, A. A. Use of b-spline surface to model large-deformation continuum plate: procedure and application. Nonlinear Dynamics (2013). 
[57] Nada, A. A., B. A. Hussein, S. M. M., and Shabana, A. A. Use of floating frame of reference formulation in large deformation analysis: experimental and numerical validation. Proceedings of the Institution of Mechanical Engineers, Part K: Journal of Multi-body Dynamics 224 (2009), 45-58.

[58] NASTRAN. version 2013.1 Quick Reference Guide. MSC.Software Corporation, Ann Arbour, MI, USA, 2012.

[59] Nielsen, J. D., Madsen, S. B., Hyldahl, P., And Balling, O. Dynamic analysis of offshore oil pipe installation using the absolute nodal coordinate formulation. Proceedings of the ASME 2013 International Mechanical Engineering Congress \& Exposition IMECE2013 (2013). San Diego, California, USA.

[60] Nikravesh, P. E. Computer Aided Analysis of Mechanical Systems. Prentice Hall, Englewood Cliffs, 1988.

[61] Niondson, F. I. Shell Theory. Elsevier Science Publishers B.V, Amsterdam, 1985.

[62] Olshievskiy, A., Dmitrochenko, O., Dai, M. D., and Kim, C.W. The simplest 3-, 6- and 8-noded fully-parameterized ancf plate elements using only transverse slopes. Multibody System Dynamics 34 (2015), 23-51.

[63] Omar, M. A., Shabana, A. A., Mikkola, A., Loh, W.-Y., And BAsCH, R. Multibody system modeling of leaf springs. Journal of Vibration and Control 11 (2004), 1601-1638.

[64] Pawsey, S. F., and Clough, R. W. Improved numerical integration of thick shell finite elements. International Journal for Numerical Methods in Engineering 3 (1971), 575-586.

[65] Polit, O., Touratier, M., ANd Lory, P. A new eight-node quadrilateral shear-bending plate finite element. International Journal for Numerical Methods in Engineering 37 (1994), 387-411.

[66] Sanborn, G. G., Choi, J., and Choi, J. H. Curve-induced distortion of polynomial space curves, flat-mapped extension modeling, and their impact on ancf thin-plate finite elements. Multibody System Dynamics 26 (2011), 191-211. 
[67] SCHIEHLen, W. Multibody system dynamics: Roots and perspectives. Multibody System Dynamics 1 (1997), 149-188.

[68] Schreurs, P., Veldpas, F., and Brekelmans, W. Simulation of forming processes, using the arbitrary eulerian-lagrangian formulation. Computer Methods in Applied Mechanics and Engineering 58 (1986), 19-36.

[69] Schwab, A. L., Gerstmayr, J., and Meijaard, J. P. Comparison of three-dimensional flexible thin plate elements for multibody dynamic analysis: Finite element formulation and absolute nodal coordinate formulation. Proceedings of the ASME 2007 International Design Engineering Technical Conferences \& Computers and Information in Engineering Conference (2007). Las Vegas, Nevada, USA.

[70] Schwertassek, R., Wallrapp, O., and Shabana, A. A. Flexible multibody simulation and choice of shape functions. Nonlinear Dynamics 20 (1999), 361-380.

[71] Seo, J.-H., KIm, S.-W., Jung, I.-H., PARK, T.-W., MoK, J.-Y., Kim, Y.-G., AND ChAI, J.-B. Dynamic analysis of a pantograph catenary system using absolute nodal coordinates. Vehicle System Dynamics 44 (2006), 615-630.

[72] Shabana, A. A. An absolute nodal coordinate formulation for the large rotation and deformation analysis of flexible bodies. Technical Report No. MBS96-1-UIC, Department of Mechanical Engineering, University of Illinois at Chicago, March 1996.

[73] Shabana, A. A. Definition of the slopes and the finite element absolute nodal coordinate formulation. Multibody System Dynamics 1 (1997), 339-348.

[74] Shabana, A. A. Flexible multibody dynamics: Review of past and recent developments. Multibody System Dynamics 1 (1997), 189-222.

[75] Shabana, A. A. Dynamics of Multibody Systems, $3^{\text {rd }}$ ed. Cambridge University Press, New York, 2005. 
References

[76] Shabana, A. A. Computational Dynamics. John Wiley \& Sons, West Sussex, 2010.

[77] Shabana, A. A. Computational Continuum Mechanics, $2^{\text {nd }}$ ed. Cambridge University Press, New York City, NY, 2012.

[78] Shabana, A. A., And Christensen, A. P. Three dimensional absolute nodal coordinate formulation: Plate problem. International Journal for Numerical Methods in Engineering 40 (1997), 2775-2790.

[79] Shabana, A. A., and Yakoub, R. Three dimensional absolute nodal coordinate formulation for beam theory: Theory. Journal of Mechanical Design 123 (2001), 606-613.

[80] Simo, J. C., AND Fox, D. D. On a stress resultant geometrically exact shell model. part i: Formulation and optimal parametrization. Computer Methods in Applied Mechanics and Engineering 72 (1989), 267-304.

[81] Simo, J. C., AND RifaI, M. S. A class of mixed assumed strain methods and the methods of incompatible modes. International Journal for Numerical Methods in Engineering 29 (1990), 1595-1638.

[82] Simo, J. C., Rifai, M. S., ANd Fox, D. D. On a stress resultant geometrically exact shell model. part iv: Variable thickness shells with through-the-thickness stretching. Computer Methods in Applied Mechanics and Engineering 81 (1990), 91-126.

[83] Simo, J. C., AND Vu-Quoc, L. On the dynamics of flexible beams under large overall motion - the plane case: Part i. Journal of Applied Mechanics 53 (1986), 849-854.

[84] Simo, J. C., AND Vu-Quoc, L. On the dynamics of flexible beams under large overall motion - the plane case: Part ii. Journal of Applied Mechanics 53 (1986), 855-863.

[85] Song, J. O., ANd Haug, E. J. Dynamic analysis of planar flexible mechanisms. Computer Methods in Applied Mechanics and Engineering 24 (1980), 359-381.

[86] Sopanen, J., And Mikкola, A. Description of elastic forces in absolute nodal coordinate formulation. Nonlinear Dynamics 34 (2003), 53-74. 
[87] Sugiyama, H., and Shabana, A. A. Application of plasticity theory and absolute nodal coordinate formulation to flexible multibody system dynamics. ASME Journal of Mechanical Design 126 (2004), 478-487.

[88] Sugiyama, H., and Shabana, A. A. On the use of implicit integration methods and the absolute nodal coordinate formulation in the analysis of elasto-plastic deformation problems. Nonlinear Dynamics 37 (2004), 245-270.

[89] Sugiyama, H., and Suda, Y. Multibody system modeling of leaf springs. Journal of Vibration and Control 223 (2009), 211-219.

[90] SzE, K. Y. Three-dimensional continuum finite element models for plate/shell analysis. Progress in Structural Engineering and Materials 4 (2002), 400-407.

[91] Timoshenko, S. P., ANd Woinowsky-Krieger, S. Theory of Plates and Shells, $2^{\text {nd }}$ ed. McGraw-Hill Book Company, New York, 1959.

[92] Valkeapä̈̈, A. I., Yamashita, H., Jayakumar, P., AND SugIYAmA, $\mathrm{H}$. On the use of elastic middle surface approach in the large deformation analysis of moderately thick shell structures using absolute nodal coordinate formulation. Nonlinear Dynamics 80 (2015), 1133-1146.

[93] Veubeke, B. F. D. The dynamics of flexible bodies. International Journal of Engineering Science 14(10) (1976), 895-913.

[94] Vu-Quoc, L., AND TAN, X. G. Optimal solid shells for non-linear analyses of multilayer composites. i. dynamics. Computer Methods in Applied Mechanics and Engineering 192 (2003), 1017-1059.

[95] Vu-Quoc, L., AND TAN, X. G. Optimal solid shells for non-linear analyses of multilayer composites. i. statics. Computer Methods in Applied Mechanics and Engineering 192 (2003), 975-1016.

[96] Waller, M. D. Vibrations of free square plates: part i. normal vibrating modes. Proceedings of the Physical Society 51(5) (1939), 831-844. DOI 10.1088/0959-5309/51/5/312. 
References

[97] Wasfy, T. M., ANd Noor, A. K. Computational strategies for flexible multibody systems. Applied Mechanics Review 56 (2003), 553-613.

[98] Yamashita, H., Valkeapä̈̈, A. I., Jayakumar, P., AND SugiYama, H. Continuum mechanics based bilinear shear deformable shell element using absolute nodal coordinate formulation. Journal of Computational and Nonlinear Dynamics 10(5) (2015), 1133-1146. DOI 10.1115/1.4028657.

[99] Yang, C. J., Hong, D. F., Ren, G. X., and Zhao, Z. H. Cable installation simulation by using a multibody dynamic model. Multibody System Dynamics (2013). DOI 10.1007/s11044-0139364-9.

[100] Yang, H. T. Y., Saigal, S., Masud, A., and Kapania, R. K. A survey of recent shell finite elements. International Journal for Numerical Methods in Engineering 47 (2000), 101-127.

[101] Zienkiewicz, O. C., ANd Taylor, R. L. The Finite Element Method For Solid \& Structural Mechanics, $5^{\text {th }}$ ed. Butterworth-Heineman, Oxford, 2000.

[102] Zienkiewicz, O. C., Taylor, R. L., and Too, J. M. Reduced integration technique in general analysis of plates and shells. International Journal for Numerical Methods in Engineering 3 (1971), 275-290. 
References 


\section{Part IV}

\section{Papers}





\section{Paper A}

\section{A thin plate element based on the combined arbitrary Lagrange-Euler and absolute nodal coordinate formulation}

The paper presented in this chapter has been published as a journal publication.

[38] Hyldahl, P., Mikкola, A. M., And Balling, O. A thin plate element based on the combined arbitrary lagrange-euler and absolute nodal coordinate formulations. Proceedings of the Institution of Mechanical Engineers, Part K: Journal of Multi-body Dynamics 227 (2013), 211-219

The content of this chapter has been excluded due to copyright restrictions but can be obtained though the respective publisher. 


\section{Paper B}

\section{Behavior of thin rectangular ANCF shell elements in various mesh configurations}

The paper presented in this chapter has been published as a journal publication.

[40] Hyldahl, P., Mikkola, A. M., Balling, O., and Sopanen, J. T. Behavior of thin rectangular ancf shell elements in various mesh configurations. Nonlinear Dynamics 78 (2014), 12771291

The content of this chapter has been excluded due to copyright restrictions but can be obtained though the respective publisher. 


\section{Paper C}

\section{Studies on the kinematics of thin shell elements based on the absolute nodal coordinate formulation}

The paper presented in this chapter has been presented at a conference and published in the conference proceedings.

[39] Hyldahl, P., Mikkola, A. M., and Balling, O. Studies on the kinematics of thin shell elements based on the absolute nodal coordinate formulation. Proceedings of the ASME 2005 International Design Engineering Technical Conferences \& Computers and Information in Engineering Conference (2014). Buffalo, New York, USA

The content of this chapter has been excluded due to copyright restrictions but can be obtained though the respective publisher. 


\section{Paper D}

\section{Dynamic analysis of offshore oil pipe installation using the absolute nodal coordinate formulation}

The paper presented in this chapter has been presented at a conference and published in the conference proceedings.

[59] Nielsen, J. D., Madsen, S. B., Hyldahl, P., and Balling, O. Dynamic analysis of offshore oil pipe installation using the absolute nodal coordinate formulation. Proceedings of the ASME 2013 International Mechanical Engineering Congress $\mathcal{E}$ Exposition IMECE2013 (2013). San Diego, California, USA

The content of this chapter has been excluded due to copyright restrictions but can be obtained though the respective publisher. 


\section{Paper E}

\section{Convergence characteristics of thin ANCF shell elements in arbitrary and initially curved mesh}

The paper presented in this chapter has been presented at a conference and published in the conference proceedings.

[41] Hyldahl, P., Mikkola, A. M., Balling, O., and Sopanen, J. T. Convergence characteristics of thin ancf shell elements in arbitrary and initially curved mesh. Proceedings of The 3rd Joint International Conference on Multibody System Dynamics $\mathcal{E}$ The 7th Asian Conference on Multibody Dynamics (2014). BEXCO, Busan, Korea

The content of this chapter has been excluded due to copyright restrictions but can be obtained though the respective publisher. 


\section{Paper F}

\section{Modeling and feasibility study of nonlinear suspension components in multibody systems using absolute nodal coordinate formulation based beam elements - application to stabilizer bar.}

The paper presented in this chapter has been presented at a conference and published in a journal.

[37] Hyldahl, P., Andersen, S., Mikkelsen, S., and Balling, O. Modeling and feasibility study of nonlinear suspension components in multibody systems using absolute nodal coordinate formulation based beam elements -application to stabilizer bar. SAE International Journal of Passenger Cars - Mechanical Systems 8(2) (2015)

The content of this chapter has been excluded due to copyright restrictions but can be obtained though the respective publisher. 